\title{
AEC POLICY AND ACTION PAPER ON CONTROLLED THERMONUCLEAR RESEARCH
}

U.S. ATOMIC ENERGY COMMISSION Division of Technical Information 
This report has been reproduced directly from the best available copy.

Printed in USA. Price $\$ 6.00$. Available from the Clearinghouse for Federal Scientific and Technical Information, National Bureau of Standards, U. S. Department of Commerce, Springfield, Virginia 22151. 


\section{DISCLAIMER}

This report was prepared as an account of work sponsored by an agency of the United States Government. Neither the United States Government nor any agency Thereof, nor any of their employees, makes any warranty, express or implied, or assumes any legal liability or responsibility for the accuracy, completeness, or usefulness of any information, apparatus, product, or process disclosed, or represents that its use would not infringe privately owned rights. Reference herein to any specific commercial product, process, or service by trade name, trademark, manufacturer, or otherwise does not necessarily constitute or imply its endorsement, recommendation, or favoring by the United States Government or any agency thereof. The views and opinions of authors expressed herein do not necessarily state or reflect those of the United States Government or any agency thereof. 


\section{DISCLAIMER}

Portions of this document may be illegible in electronic image products. Images are produced from the best available original document. 


\section{UNITED STATES \\ ATOMIC ENERGY COMMISSION \\ WASHINGTON, D.C. 20545}

July 11, 1966

Dear Mr. Holifield:

The Joint Committee on Atomic Energy has requested that the Commission develop a report on its Controlled Thermonuclear Program, similar to the Policy Paper prepared on the High Energy Physics Program.

In May 1965 the Atomic Energy Commission formed a pane1 of leading scientists and engineers to carry out a detailed review of its program on Controlled Thermonuclear Research. The work of that panel was completed in December 1965, and a copy of its report was transmitted to the Joint Committee on Atomic Energy on January 3, 1966. In the period since the Review Panel's report, the Commission has completed a further study and has prepared the attached report entitled, "AEC Policy and Action Paper on Controlled Thermonuclear Research."

Both the Review Panel and the Commission have concluded that an intensification of effort is needed. To ensure that this is done in full consideration of other program requirements, both within the AEC and the government as a whole, the needs of the controlled fusion program were reviewed by the AEC's General Advisory Committee and the President's Science Advisory Committee. After their endorsement of the importance of the program and the need for an intensified effort, the Commission has adopted the attached report, which will serve as a planning guide for the future development of the CTR program. The Commission, of course, will review the CTR program annually with the Bureau of the Budget and the Congress to determine the appropriate level of activity which should be maintained in this important field of endeavor.

The Bureau of the Budget has reviewed this report and expresses no objection to its transmittal. However, the Bureau has pointed out that the degree of program growth is subject to annual budget review.

Cordially,

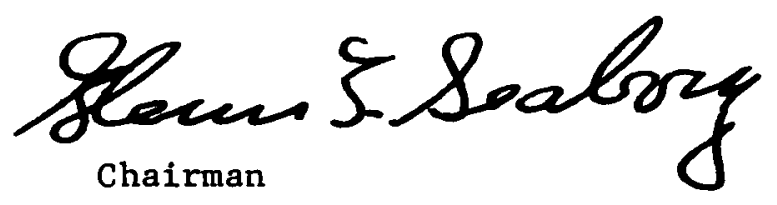

Honorable Chet Holifield, Chairman Joint Committee on Atomic Energy 


\section{AEC POLICY AND ACTION PAPER ON CONTROLLED THERMONUCLEAR RESEARCH}

JUNE 1966

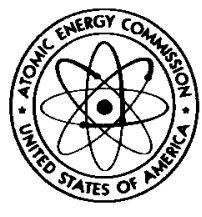

UNITED STATES ATOMIC ENERGY COMMISSION

Division of Technical Information 


\begin{abstract}
Addendum to
TW-23277, AEC Policy and Action Paper on Controlled Thermonuclear Research

(This acknowledgment page, which was included in the preprint copies, was inadvertently omitted from the printed report.)
\end{abstract}

\title{
ACKNOWLEDGMENT
}

The Commission wishes to express its appreciation to the Project Directors of the four CTR Laboratories (i.e., Drs. M. B. Gottlieb of Princeton, A. H. Snell of Oak Ridge, R. S. Taschek of Los Alamos, and C. M. Van Atta of the Lawrence Radiation Laboratory) for their assistance in preparing certain sections of this document, particularly Appendix 3 and part of Section III. It is especially indebted to Dr. R. F. Post for his willingness to participate actively in drafting this report (particularly Section II). Sincere thanks are also given to members of the Review Panel,* and especially to its chairman, Professor R. G. Herb, for their many contributions relating to this document.

* Names of Panel Members are given in Section IV. 
AEC POLICY AND ACTION PAPER ON

CONTROLLED THERMONUCLEAR RESEARCH

CONTENTS

SECTION I. ORIGIN AND PURPOSE OF THIS DOCUMENT.......... I-I

SECTION II. $\quad$ SCIENTIFIC BACKGROUND................. II-I

A. Nature of the Problem................ II-I

B. Attacking the Problem................ II-2

C. The Concept of Magnetic Confinement........ II-6

D. Closed or Open-Ended.$\ldots \ldots \ldots \ldots \ldots \ldots \ldots$ II-7

Toroidal Confinement................ II-7

The Stellarator and Rotational Transform.. II-9

Open-Ended Bottles - The Mirror Machine... II-10

The Astron Concept................. II-Il

Other Possibilities............... II-12

E. Creating the Plasma................. II-J2

F. Loss Processes and Plasma Instabilities...... II-14

Radiation and Charge Exchange............ II-14

Collisional Diffusion................. II-15

Plasma Instability................ II-16

G. Pulsed or Steady-State? The Question of

Plasma Density...................... II-18

H. Minimum-B and Average Minimum-B........... II-20

I. Technological Developments............... II-23

J. "The Shape of Things to Come"............ II-25

K. "The Fusion Handbook"................ II-30 
Page

SECTION III. STATUS OF RESEARCH OR PLASMA PHYSICS AND

CONTROLLED FUSION . . . . . . . . . . . III-I

A. Research in the United States . . . . . . III-I

Research in AEC Laboratories . . . . . III-2

Iawrence Radiation Laboratory . . . . . III-2

The Magnetic Mirror Program ...... III-3

ASTRON . . . . . . . . . . . III-6

Toroidal Confinement . . . . . III-8

Ios Alamos Scientific Iaboratory . . . . III-9

Scylla IV . . . . . . . . . . . III-1I

Dense Plasma Focus . . . . . . . III-12

Hydromagnetic Plasma Gun .. . . . . . . III-12

Mathematical and Theoretical Physics . . III-13

Oak Ridge National Laboratory . . . . . III-14

Molecular Ion Injection, DCX-1 .... III-14

Molecular Ion Injection, DCX-2 . . . . III-15

Electron Cyclotron Heating . . . . . III-16

Beam-Plasma Interaction . . . . . . III-17

Princeton Plasma Physics Laboratory . . . III-18

Research at Universities . . . . . . III-22

University of California at San Diego . . III-24

New York University . . . . . . . . . III-24

University of Tennessee . . . . . . . III-25

University of Michigan . . . . . . . III-25

Georgia Institute of Technology . . . . III-25

Swarthmore College . . . . . . . . III-25

Yale University . . . . . . . . . . III-25

MIT . . . . . . . . . . . . . III-25

Stanford University . . . . . . . III-25

University of Wisconsin . . . . . . . III-25

Stevens Institute of Technology . . . . III-26

Plasma Research Programs or Other Government

Agencies . . . . . . . . . . . III-26

Naval Research Laboratory . . . . . . III-27

NSF Support at MIT . . . . . . . . III-27

A1r Force Support at Asrojet-General . . . III-27 
Page

Plasma Research by Industry . . . . . . III-28

General 1tomic . ......... III-29

General filectric . . . . . . . III-29

United Aircraft . . . . . . . . III-30

Westinghouse ......... III-30

B. Foreign Programs . . . . . . . . III-30

U.S.S.R. . . . . . . . . . . III-3I

United Kingdom . . . . . . . . . . . III-33

West Germany .......... . . III-35

France ............. . III-36

Japan . . . . . . . . . . . III-36

Other Nations . . . . . . . . . III-37

International Cooperation . . . . . . III-37

C. Summary of Progress ........... III-38

SECTION IV. REV IEWS OF THE PROGRAM BY EXTERNAL COMMTTTEES . . IV-1

1962 Report of the General Advisory Committee . . . IV-1

1965 Review Panel ............. . IV-3

1966 Report of the Subpanel on Plasma Physics,

National Academy of Sciences ....... . IV -5

SECTION V. EVALUATION OF THE OVERALI EFFORT ON CONTROLLED

FUSION ................ $\mathrm{V}-\mathrm{l}$

A. Evaluation of the Importance of the Program . . V-1

B. Evaluation of the Present Status of the

U. S. Effort ........... V V-3

1. General ............ . V-3

2. Princeton Plasma Physics Laboratory ... . V-5

3. Oak Ridge National Laboratory . . . . . V-6

4. Ios Alamos Scientific Iaboratory .... V V-7 


\section{$\underline{\text { Page }}$}

5. Lawrence Radiation Laboratory . . . . . V-8

6. Non-AEC Program ........... . V-10

7. AEC's University Program ........ V-10

C. Comparison of U.S. and the World Effort . . . V-II

D. Existing Budgetary Considerations and their

Consequences ............ V V-12

Impact on Manpower and Programs . . . . . V-12

New and Neglected Areas ......... V V-13

E. Considerations of a National Center . . . . V-15

F. Overall Conclusions . . . . . . . . V-16

Scientific-Technical ........ V V-16

Administrative .......... . . V-18

Implications ............ . . V-19

SECTION VI. PLANNED ACTION . . . . . . . . . . . . VI-I

A. Policy Statements ....... . . . . . VI-I

B. Specific Steps to be Taken .. . . . . . . VI-2

1. Concerming the AEC's Program as a Whole . . VI-2

(a) Scientific-Technical. . . . . . VI-2

(b) Administrative . . . . . . . VI-3

(c) Financial . . . . . . . . VI-3

2. At the Princeton Plasma Physics Laboratory. VI-5

3. At the Oak Ridge National Laboratory . . VI-6

4. At the Los Alamos Scientific Laboratory . . VI-7

5. At the Lawrence Radiation Laboratory . . VI-7

6. Regarding Work at Non-AEC Sites . . . . VI-8

7. Regarding AEC-Supported Work at Universities . . . . . . . . . VI-8

8. Regarding the National Center ... . . VI-8 


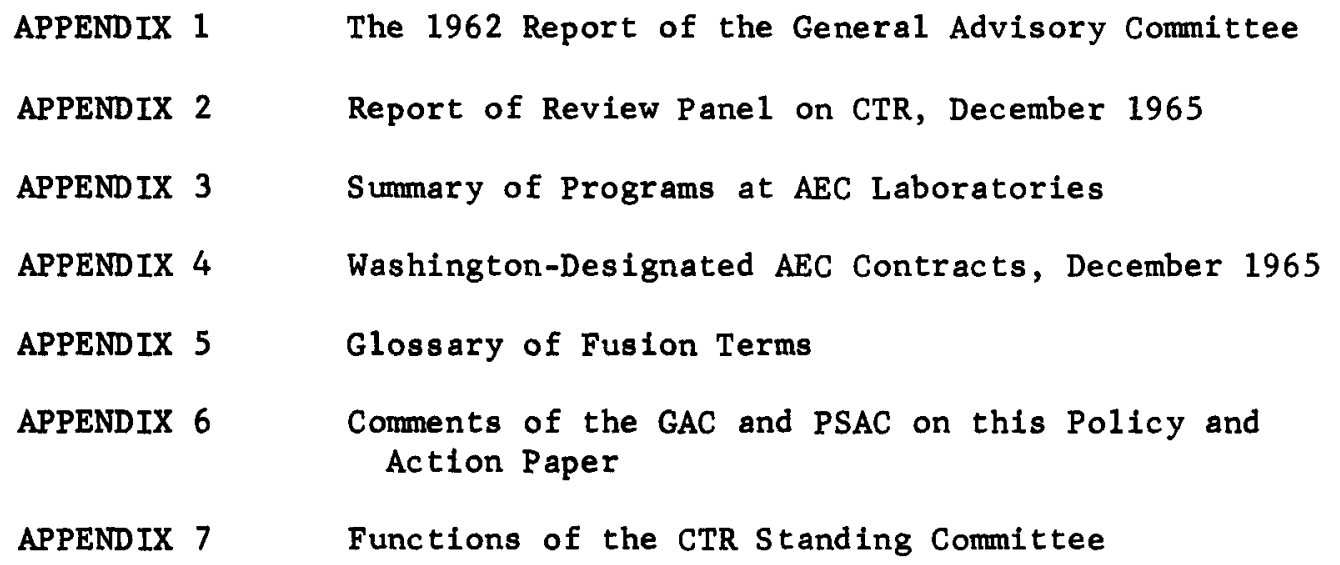


SECTION I. ORIGIN AND PURPOSE OF THIS DOCUMENT

During 1965, the Atomic Energy Commission undertook a major review of its research program on controlled thermonuclear reactions. In doing so, the Commission was motivated by the desire (I) to carry out an evaluation in depth of the present status and future prospects of its work in this field, and (2) to provide sound answers to penetrating questions raised by members both of the Joint Committee on Atomic Energy and of the House Appropriations Committee.

In connection with this review, the Joint Committee on Atomic Energy requested the Commission use its findings and conclusions as a basis for developing a Policy, Paper on the controlled thermonuclear program, similar to that prepared on the high energy physics program. The present document is in specific response to this request.

Section II of this document provides a review of the scientific background for the CTR program, including the nature of the problem, the methods of attack, etc. The material of this section serves as an introduction to the technical discussions given later in the paper.

Section III gives a brief survey of the present status of research in controlled fusion, both in this country and abroad. A more detailed presentation of fusion research at the AEC-supported sites is given in Appendices 3 and 4. 
Section IV gives a brief summary of the various reviews of the AEC's fusion program by external committees. The latest of these reviews, carried out in 1965 under the chairmanship of Professor S. K. Allison (and after his death, under the chairmanship of Professor R. G. Herb) has been especially helpful to the Commission in evaluating its own program and in the preparation of the present document.

Section $V$ presents the evaluation by the Commission of the overall effort in the controlled thermonuclear field, including a comparision of the work in this country and abroad, together with considerations evolving from this comparison..

In conclusion, Section VI states the proposed Commission action relating to the AEC effort in this field. This proposed action consists of two parts: (1) the establishment of policy designed to guide the development of the program, and (2) a set of specific steps which serve to implement this policy. 
SECTION II. SCIENTIFIC BACKGROUND

The objective of controlled fusion research is to provide mankind with a new source of energy. Despite the applied nature of 1 ts goal, at the present time progress toward this goal depends critically upon the performance of fundamental studies. In this regard fusion research resembles other areas of basic physics research and, in common with these fields, it is impossible to predict in detail the outcome of the research.

The following material is intended to provide a review of the salient scientific features of the controlled fusion problem in as descriptive a manner as possible. In it will also be found concepts and definitions of terms useful in understanding the fusion research programs described in Section III.

\section{A. The Nature of the Problem}

At first sight, the requirements for achieving useful power from sustained thermonuclear reactions stagger the imagination. They are: (a) to heat a dilute gas of fusion fuel to temperatures of hundreds of millions of degrees, (b) to contain it, free from any contact with material walls or from contamination by impurities, long enough for an appreciable fraction of the fuel to react, and finally, (c) to extract the energy thus released and convert it to a useful form. Not only must this scientific legerdemain be performed with finesse but, to be useful, it must obviously be accomplished with an expenditure of energy substantially smaller than 
that released by the fusion reactions themselves. Eventually, the whole process must be made compatible with economic requirements, both as to cost and size.

\section{B. Attacking the Problem}

To see how the fusion problem can be attacked, consider the nature of the fusion process itself. Nuclear fusion reactions are "rearrangement reactions" that occur between nuclei of energy-rich light isatapes, such as deuterium or tritium (heavy 1sotopes of hydrogen). For such reactions to occur two nuclei must "collide", that is, they must approach each other within a distance about equal to their own diameter, so that rearrangement, forming stabler nuclei, can occur. The resulting nuclei are less massive than the original pair and, as predicted by Einstein's mass-energy relationship, this difference in mass is released as kinetic energy, 1.e., the new pair is expelled from the scene of the reaction with increased energy of motion. The most striking features of fusion reactions as a source of energy are the ready and universal availability, the low cost, and the essential inexhaustibility (billions of years' supp1y) of the primary fuel, deuterium. Deuterium occurs naturally, mixed with ordinary hydrogen, in a concentration of about $1: 6000$ and is readily and cheaply separated from water.

The nuclear fusion reactions involving deuterium alone (D-D or "deuteron-deuteron" reactions) are not the only ones of interest. others include the D-T (deuterium-tritium) and the D-Helium-3 
reaction (Helium-3 is a light isotope of helium). In fact, in speculating about fuels that might be used for fusion power, D-T is a prime candidate. It has the great advantage that both the probability of reaction and the energy release per reaction are much larger for $D-T$ than the corresponding numbers for the $D-D$ reaction. Since tritium does not occur in nature, any system using the D-T reaction would have to include a regenerating blanket in which the neutron released from the D-T reaction would be captured (probably in lithium, also abundant and cheap) to produce more tritium. Studies have shown that this process should be feasible, both technically and economically.

On the other hand, if the $D-D$ reaction could ever be utilized, it would have several advantages. $D-D$ reactions result in reaction products (either tritium or helium-3) that are themselves fusion combustible. The end products are ordinary helium and hydrogen. Indeed this is a characteristic feature of fusion reactions: the end-products are non-toxic and non-radioactive. Fusion reactors would have to be shielded because of neutrons released, but no pollution, waste disposal, or reactor runaway hazard would result from their use.

Nuclei, being positively charged, repel each other so that fusion reactions only occur when nuclei approach each other with substantial relative energy. This required energy is roughly one percent of the energy released in the fusion reaction itself, giving the 
available "payoff" ratio for fusion reactions. The situation differs greatly from that encountered in the fission of uranium by neutrons. A neutron, being uncharged, can readily penetrate a uranium nucleus and cause fission, even when its energy of motion is microscopic compared to that released.

In nature, that is in the sun and the stars, nuclear fusion reactions are said to be of "thermonuclear" origin, meaning that they occur as a result of the extremely high temperatures existing in the stellar core. High temperature implies rapid motion of the nuclei of which the star is composed. This random motion promotes collisions and fusion reactions. "Confinement" against loss of the thermonuclear fuel before it can react is provided by the intense gravitational field of the star.

On earth one cannot hope completely to duplicate a star's prescription for sustained fusion reactions. Another approach must be sought. Fortunately, the very fact that fusion requires high temperatures opens up a novel possibility: All matter raised to a sufficiently high temperature will be broken down to the plasma state -- a gas composed of charged particles - as a result of the violent collisions that "high temperature" implies. Though, as a whole, a plasma is electrically neutral, i.e., equal numbers of negative and positive charges, its constituent particles can be controlled by an electromagnetic field. The guiding of high energy particles by the magnetic fields of a particle accelerator is an example of the 
control of charged particles. From this general concept evolved the idea of magnetic confinement: the hot plasma is to be contained in space through control of its individual particles by means of a magnetic field, shaped so as to form a kind of "magnetic bottle." Though generically related to the idea of the guide field of a particle accelerator, the problem of particle control in fusion appears at a different level. First, even though the particle energies are much lower (tens of thousands of electron volts for fusion as opposed to millions or even billions of electron volts in particle accelerators), both the total number and the number per unit volume of particles that must be controlled is vastly greater. Second, whereas the particles in an accelerator move head-to-tail along prescribed orbits, neither appreciably influencing each other's motion nor disturbing the guiding field, the particles in a fusion reactor must move within the chamber in random directions, to promote the probability of mutual collisions. Also, because the particle density would have to be much higher in a fusion reactor than it is in an accelerator, the plasma particles themselves might, through cooperative motions, generate electric or magnetic fields that could affect the particles' own confinement.

The last point mentioned, cooperative effects, hints at the salient question for all of fusion research today: How can a magnetic bottle be made, whose effectiveness is not spoiled by the very presence 
of the plasma, 1.e., how can a plasma be magnetically confined and yet not break into spontaneous unstable motions that spoil the confinement?

\section{The Concept of Magnetic Confinement}

If plasma instability can be adequately controlled, theory indicates that magnetic bottles should be ideally suited to solve the containment problem. To visualize the idea of magnetic confinement, first consider what would happen to a thermonuclear plasma were it suddenly established within a long cylindrical tube, otherwise evacuated. A snapshot of such a plasma would reveal that its particles are all moving in straight paths, but in random directions and at very high speeds (over 1000 miles per second for the deuterium nuclei, and much faster than this for the electrons). Without means for confinement this speed would be sufficient to carry all of the particles to the wall in less than a millionth of a second. But now, if a magnetic field were to be established inside the tube by passing electrical current through a magnet coil wound around it, something very different would occur. In this case, the direction of the magnetic field, visualized by lines of magnetic force or by the direction a compass needle could point, is parallel to the axis of the tube. In such a field the charged particles could in general no longer move in randomly directly straight paths, but would instead be constrained to move in the direction of the lines of force (axially) following tightly wound (coil spring-1ike) trajectories.

$$
\text { II }-6
$$


If sufficiently intense, such a magnetic field would provide a very effective degree of containment, as far as sideways motion of the plasma particles is concerned.

Unfortunately, the simple system just described could not function as an effective magnetic bottle. Since a magnetic field exerts a force that is perpendicular to its direction (paralle1 to the axis of the tube), a field such as here described could not prevent the plasma from rapidly leaking out the end of the tube, much as water escapes from an open pipe. Something must yet be added to the picture to produce a magnetic bottle.

\section{Closed or Open-ended?}

The conversion of the concept of magnetic confinement to reality involves both a choice and an inventive step. The reason for this is exemplified by the failure of a uniform magnetic field, directed along a cylindrical tube, to do more than prevent the plasma from escaping transversely. Two fundamentally different ways present themselves for resolving this dilemma: (1) The magnetic field may be caused to close on itself by bending the tube, and the magnetic coil wound around it, into a circle to form a torus, i.e., a doughnut-shaped chamber. (2) The ends of the open tube may somehow be provided with magnetic stoppers to prevent the escape of particles.

Toroidal Confinement. Consider now the closed tube. Here the magnetic lines are all contained within the tube. The particles, 
moving along these lines in their helical trajectories, seemingly

cannot escape. However, the simple toroidal magnetic bottle

possesses a fundamental flaw, a flaw that prevents it from confining

a plasma appreciably longer than if no magnetic field at all were present. The origin of the flaw hints at the subtlety of magnetic confinement. The key point is this: whenever the lines of force of a magnetic field are bent, i.e., the direction of the magnetic field changes in space, then the strength of the magnetic field can no longer be uniform. A simple example is the magnetic field surrounding a straight wire carrying a current. Here the magnetic field lines are circles surrounding the wire, and the intensity of the field falls off as one moves away from the wire. The example also illustrates another point, valuable for understanding magnetic confinement: The local strength of a magnetic field aways weakens in directions in which the magnetic lines of force are concave, i.e., bulge away from the observer. Conversely, the field always increases in strength in directions in which the field lines are convex, that is bulge toward the observer.

Here then is the flaw in the simple toroidal magnetic bottle: bending a cylindrical tube and its surrounding magnet winding into a torus creates a magnetic field inside the tube with lines of force that are circles. Thus the lines of force will appear concave looking from the inside toward the outer wall of the torus. This means that the magnetic field strength decreases in this direction. Suppose one attempts to use such a field to confine a plasma. 
Magnetic confinement implies a balance between (a) the magnetic forces exerted inward on the plasma and (b) the plasma pressure, arising from its tendency to expand (as any gas tends to expand). But in the simple torus there can be no such pressure balance. By simply expanding toward the outer wall of the torus, the plasma will find itself opposed by even weaker magnetic forces, thus it may more readily expand, and so forth, and so forth, until it strikes the outer wall. This resembles what would happen if one attempted to contain high pressure gas in a vessel, one wall of which became progressively weaker with outward movement. The result would be an explosively rapid escape of gas.

The Stellarator and Rotational Transform. One aspect of the trouble with the simple torus is that particles of the plasma that are moving in their helical paths in the vicinity of the outer wall spend a11 of their time in regions where the plasma drift is toward the wall, whereas particles streaming along near the inner wall of the chamber spend their time in fields where the drift is away from the wall. The invention of the stellarator solved this problem. The idea of the stellarator is to arrange matters so that the particles, in their flow along the lines of force, spend part of their time nearer the outer wall, but also part of their time nearer the inner wall. Thus if they are moving rapidly enough along the field lines their drifts, being alternately toward and away from the wal1, will just average to zero. The way this trick was first accomplished was to bend the tube into a Figure 8 . 
It was later found to be just as effective to use extra windings, in addition to the regular magnet coil windings, to accomplish the "twist."

When these special windings, which take the form of longitudinal conducting bars, are energized, the effect is to cause the field lines, formerly circular, to become helical. The magnitude of the field twisting effect produced is called the degree of "rotational transform." (In a single torus the rotational transform is zero.) The particles, now spiralling about and guided by these helical lines spend time alternatively near the outer and then the inner chamber walls, thus experiencing the averaging effect mentioned above. This is the basic idea of the Stellarator.

Open-Ended Bottles - The Mirror Machine. It was noted earlier that another possible method of solving "the problem of the ends" would be to provide some kind of magnetic stopper that would keep the fusion fuel ions from escaping out of the ends of the tube before they had a chance to collide and react. Fortunately, there exists an effect, known for many years, that $c$ an be used to make a magnetic bottle out of a straight tube. This is the so-called "magnetic mirror" effect. The name refers to the fact that a charged particle, spiraling along a magnetic line of force, will generally be "reflected", i.e., its component of motion along the line of force will be stopped and then reversed, if it encounters a region of stronger-than-average magnetic field. A mirror machine is therefore simply a confining field of roughly tubular geometry, capped at 
both ends by locally strengthening the field. The earth's

magnetic field, above the stratosphere, is an example of one such field, a terrestrial "mirror machine" where there exists the Van Allen radiation belts, plasmas of natural origin.

A mirror machine is said to be an "open-ended" magnetic bottle because the magnetic lines of force, though tightly converged at the mirrors, actually pass through the mirrors, diverge, and then pass out the ends of the chamber. For this reason, there always exists a small leak path for particles along the field lines and therefore the containment of particles in a mirror machine can never be as perfect as it would be in an ideally operating stellarator. There are, however, compensating advantages to open-ended systems that will be 1 ater discussed.

The Astron Concept. Many of the advantages of open and closed magnetic bottles may be obtained in a single bottle by means of the Astron concept. The Astron combines the magnetic field of a simple mirror bottle with the magnetic field created by a very strong cylindrical current of high-energy electrons coaxial to the coils that form the mirror. The combination of the two fields should convert the initially open-ended field pattern to one of closed lines of force. The energetic electrons travelling through the trapped plasma lose energy by collisions and by this means are expected to heat the plasma to fusion temperature. 
other Possibilities. Though all magnetic bottles, of whatever form, must fall into one of the two categories, open or closed, the examples that have been given do not exhaust the possibilities. In an abbreviated discussion of this kind it is not possible to mention all of the hybrid systems or variations on the basic themes that have been proposed or are under investigation. In the several years since fusion research began, many of these ideas have fallen by the wayside. An example of this is the so-called "toroidal pinch." The idea, intuitively attractive, was to compress and confine a hot plasma by utilizing the magnetic field produced by intense electrical currents transformer-induced in the plasma itself. When tested, toroidal pinches proved to be hopelessly unstable, a fact borne out (indeed predicted) by theoretical calculations. This entire "approach" was therefore dropped, even though it at one time made up a large fraction of the world's fusion effort.

\section{E. Creating the Plasma}

Up to this point the demanding conditions for achieving fusion have been outlined - including the most demanding of all: contalnment. But even assuming that the containment problem is solved by the use of some form of magnetic bottle, there still remains the question of how to create a hot plasma inside the bottle. Though not as basic as the problem of confinement, this problem is by no means trivial. Again a dilemma: Given a magnetic bottle that is adequately leakproof against plasma escape, such a bottle will also be impervious 
to the entry of the very plasma particles one wished to contain. Solutions: one must either (a) create and heat the plasma in situ or (b) introduce the plasma particles "in disguise" (for example as energetic neutral atoms), then remove the disguise inside the bottle, or (c) weaken the magnetic field in some way so that it cannot resist entry of the plasma, then immediately strengthen it before the plasma can escape. All three of the above methods or variations of them are used in confinement experiments today. The choice of which method is best to use depends on the type of magnetic bottle being studied. For example, in the stellarator the most straightforward method has been to fill the chamber with neutral gas, ionize (break up the atoms of) this gas with a high frequency discharge, heat it "lukewarm" by passing an electrical current through it, and then finally heat to high temperatures by means of resonant excitation with powerfuI radio waves, tuned to the "cyclotron" period of rotation of the plasma ions in the confining magnetic field.

Though all three of the basic methods listed above have now been proved successful in creating plasmas of thermonuclear temperature, for years the technical problem of creating the hot plasma proved one of the most difficult elements in experimental high temperature plasma research.

An "off-beat" method of creating a hot plasma, just now being examined, uses a laser beam to produce a plasma inside a magnetic bottle. The laser beam is focussed, through a window in the side 
of the vacuum chamber, onto a tiny pellet of solid material(such as frozen deuterium) dropped through the beam or else held suspended by electrostatic forces. The concentrated energy of the laser beam vaporizes and ionizes the pellet, producing in situ a cloud of hot plasma.

\section{F. Loss Processes and Plasma Instabilities}

The key question in achieving useful power from fusion reactions is, of course, not whether or how the reactions will occur, but rather, what are the energy loss processes that compete with the fusion energy released, and could they overwhelm it unless minimized? The situation is not unlike that of the gas turbine (a compressor driven by a turbine with a combustion chamber in between) where the concept was born years before the actuality. In this case, success hinged on the development of compressors efficient enough to do their job without requiring the full power of the turbine to do it. Radiation and Charge Exchange. Energy losses in fusion can be divided Into two classes: (1) unavoidable ones and (2) ones that must be minimized by cleverness or increase in size,or both. In the first category are the radiation losses. These losses can be calculated with precision and it is known that they are tolerable, but only (a) if the plasma temperature is high enough (fusion reaction rates increase faster with increasing temperature than do radiation losses) and $(b)$ if contamination by impurity ions of higher atomic number (e.g., oxygen, or metal ions) is kept to very low levels. The 
impurity problem, though important, has fortunately been brought under control in most high temperature plasma experiments. There is another loss process, of non-plasma origin, that must be faced. Fortunately, this one becomes less important the closer thermonuclear conditions are approached. The process, called "charge exchange", may occur whenever a plasma fuel ion (e.g., a deuteron) passes in the vicinity of a neutral atom (say an impurity atom). The attractive force between the fon and a bound electron of the atom may cause the electron to be plucked off and captured by the fuel ion, thus converting the hot, confined ion to a fast moving neutral atom. No longer influenced by the magnetic field, the fast atom escapes, leaving behind a low energy impurity ion. The need to minimize charge exchange losses explains the need for extremely good vacua in fusion experiments where the plasma density is initially low. Charge exchange losses are less important in denser plasmas, which protect themselves by rapidly ionizing (through collisions with the electrons) any neutral atoms that attempt to enter. This process is sometimes called "burn out".

Collisional Diffusion. Another "unavoidable" loss process is that arising from collisions between the particles. "Collisions" are obviously essential for fusion reactions, yet not all "collisions" between fuel ions lead to reactions; some lead only to deflecting the path of the ion into a new direction. Such deflections represent a mechanism for particles to escape from a magnetic bottle. To see 
this, note that a sudden deflection in the motion of the particle will move the axis of its helical trajectory sideways, i.e., across the confining magnetic field. An accumulation of such deflections, even though randomly directed, would eventually carry the particle to the chamber wall. The theory of this kind of "collisional diffusion" in closed (toroidal) systems indicates that losses from this process can be made small compared to the fusion energy release, provided strong magnetic fields are used and the chamber diameter is not too small. Neither the strength of field nor the chamber diameter required would be impractically large. The theoretical margin is much less robust for open-ended systems, but even in this case the use of sufficiently high temperatures and sufficiently powerful mirrors should permit a favorable power balance.

Plasma Instability. Up to this point the discussion has been concerned. with a well-established body of knowledge from which a meaningful picture of fusion and its potentialities can be drawn. Yet there remains a key issue at the focus of all fusion research: plasma instabilities. Because of both its relevance and its intrinsic scientific interest, the instability problem has stimulated an almost unprecedented advance in plasma theory, coupled with a rapidly growing body of corroborative experimental evidence. The picture that has emerged can be summarized as follows:

1. Concern about plasma instabilities in magnetically confined plasmas arises because unstable behavior can lead to more rapid loss of the plasma than do the "controllable" losses mentioned above. However, it 
now appears that the effect of plasma instabilities on magnetic confinement may vary widely -- from catastrophic to innocuous - depending on the type of instability considered, and on the type of magnetic bottle employed. Control of instabilities must be adequate but apparently need not be complete.

2. Plasma instabilities $c$ an be divided into two general classes: (a) gross or "hydromagnetic" types and (b) wave-particle instabilities. As the name implies, gross instabilities involve unstable motion of the plasma as a whole across the confining field. Means for completely controlling gross instabilities in important cases have now been found and will be later discussed. Wave-particle instabilities, often called "microinstabilities", involve resonant interactions between plasma waves (electromagnetic, electrostatic, or acoustic waves) and some ordered motion of the plasma particles -- e.g., the circling of ions at their "cyclotron" frequency in the confining magnetic field. These wave-particle instabilities are therefore analogous to the resonant amplification of a light wave as it passes through the "lasing" medium.

3. Theory has provided a valuable guideline for identifying the basic sources of energy that feed plasma instabilities and for systematically reducing these "energy reservoirs." The sources relate to the extent to which a given confined plasma is not uniformly randomized, i.e., particles moving randomly. Thus density or temperature variations, or poorly randomized particle velocity distributions are all potential sources of instability. The clue to minimizing tendencies for instability is to remove or to minimize as many of these sources as possible, consistent with confinement. Though such sources can never be completely removed, this points to the value of creating well-randomized plasmas, and to the need for scale-up in size of the plasma resulting in less abrupt density variations.

Not only is the elucidation of plasma instabilities of central importance for fusion research, but it bears on other possible technological applications of plasma physics. As an example, some wave-particle interactions, that are called "beam-plasma instabilities" 
in fusion research circles, are closely related to phenomena used to practical advantage in certain ultra-high frequency electronic tubes. Furthermore, our understanding of the complicated astrophysical plasma phenomena seen both in the solar system and in outer space depends heavily on an understanding of plasma instabilities.

\section{G. Pulsed or Steady-State? The Question of Plasma Density}

In addition to the presently unresolvable choice between open-ended and closed magnetic confinement there is another area in which a choice of research emphasis can be made. In thermonuclear plasma the rate of reaction increases with increase in the plasma density, as does the pressure exerted by the plasma on the confining field. Thus, although the use of very low plasma density would greatly simplify the problem of achieving plasma stability, through reducing the plasma pressure, such "low beta"* operation would drop the thermonuclear power density which varies as the square of the particle density to uselessly low levels. Very high density, on the other hand, would greatly reduce the required confinement time but would result in a near-explosive release of fusion energy, require extremely intense magnetic fields for confinement, and could be expected to increase the probability of encountering instabilities. Between these two far extremes lie two possible operating regimes for a fusion reactor:

*Beta is defined as the ratio of plasma pressure to magnetic field pressure. In a "low beta" plasma the plasma has little influence on the magnetic field, which permeates it completely. With "high beta" plasma the magnetic field inside is much weaker than it is outside, being "pushed out" by the pressure of the plasma. 
1. "Intermediate" Densities. "Intermediate", as used here, refers to particle densities of around $10^{14}$ ions per cubic centimeter, i.e., about 1/100,000 of atmospheric densities. A fusion reactor operating at intermediate particle densities would have fusion power densities of a few megawatts per cubic meter of reacting plasma. To achieve a net energy release from such a plasma as defined by the "Lawson Criterion"*, the average ion containment time must be greater than 1 second. The plasma pressure would be modest (a few hundred pounds per square inch), so that the reactor would be one of "low beta."

2. "High" Densities. As used here, "high" refers to particle densities greater than 10I6 ions per cubic centimeter, 1.e., about 1/1,000 of atmospheric densities. A fusion reactor operating at high particle densities would have fusion power densities of about 10,000 megawatts per cubic meter of reacting plasma. To achieve a net energy release from such a plasma the average ion containment time must be greater than 0.01 second. The plasma pressure would be high (several thousand pounds per square inch), so that the reactor would be one of "high beta."

Self-sustaining reactions can, in principle, be produced over a wide range of plasma densities and confinement times, so that the choice of these quantities can

\footnotetext{
*The "Lawson Criterion" states that a minimum cọndition for a confined thermonuclear plasma to produce a self-sustaining reaction against loss of energy through particle loss, is that the product of the particle density $n$ (in ions per cubic centimeter), and the average ion containment time, $t$ (in seconds), must be larger than a certain number (typically $3 \times 10^{14}$ for a deuterium-tritium plasma).
} 
be made on the basis of other considerations. On the other hand, the temperature of a thermonuclear plasma cannot be so freely chosen. The nature of fusion reactions requires that the plasma temperature exceed about 100 million degrees centigrade (about $10 \mathrm{kilo}$ electron volts average energy of motion of the particles). If it is assumed that adequate suppression of plasma instabilities will eventually be achieved, then, based on the intermediate density operating mode suggested above, continuously-fed fusion reactors could be visualized. For example, theoretical studies of closed and open-ended systems operating in this mode were made several years ago. These studies all indicated favorable power balances, assuming stability.

It is possible that plasma stability adequate to permit long-time confinement may not be achievable using present ideas. In this case, high density, pulsed operation would be required, in order to minimize the importance of confinement time. Again, one can visualize possible operating modes along the lines of a "pulsed reactor." Only time will tell which of the two modes will be the more successful.

\section{H. Minimum-B and Average Minimum-B}

One of the most promising recent developments in fusion research has been the theoretical prediction (and experimental proof) of the gross stabilizing effect of "minimum-B" or "positive-gradient" mirror confining fields. Though the idea was first put forth in 1958, in the U.S., the USSR made the first definitive test in 1961. 
The basic idea is to arrange the coils that produce the confining field so that there exists a region between the mirrors where the field intensity has a local minimum. Plasma trapped in such a region cannot escape through gross instability, since any sideways motion of the plasma as a whole will carry it into a region of higher magnetic field. The analog is any well-designed pressure vesse1, the walls of which stiffen with any outward motion. The minimum-B idea is not only obvious but it works! Following the first demonstration, this principle has been put to work in plasma experiments throughout the world. Remembering the discussion of convex and concave lines of force, it can be seen that a requirement for forming a minimum-B region is that, looking outward in any direction from this region one must see convex lines of force. That this requirement can also be made compatible with having tightly converged lines of force (the mirror regions) at each end is not obvious, but can be made plausible by a simple demonstration: Roll a sheet of paper into a cylinder. Now strongly squeeze the cylinder at one end, forming a flattened ellipse as viewed from the end. This is one of the mirrors. Keeping the first end compressed, squeeze the other end of the cylinder so as to form another flattened ellipse at right angles (as viewed from the end) to the first one. Looking next at the resultant geometric figure (a sort of trapezoid), one can see that this figure could be generated by a set of convex field lines going along the surface 
from one end to the other. This is a rough model of a "quadrupole" (four-sided symetry) minimum-B field. A novel way by which a quadrupole minimum-B field can be produced in the laboratory is to wind the magnet coil in the shape of the seam on a baseball. Such coils have been built and are in use.

The desirable stability properties of open-end minimum-B mirror fields prompted theorists to see if a minimum-B fleld could be achieved in a torus by winding properly shaped coils around it. It was soon apparent from the geometry that it is impossible by these means to create a toroidal region that everywhere along its length has the minimum-B property. This can be seen in terms of the "convex line" aryument by noting that lines of force that close around a circular path cannot everywhere be convex (curve away from) an observer walking along them - somewhere they have to curve back to get around the bends.

Despite the impossibility of finding magnet windings that would produce true minimum-B in a torus, careful theoretical investigations showed that the next best thing could be accomplished. Thus was born the "average minimum-B" concept. In such a field a contained plasma, if it attempted to move in any direction as a whole, would encounter on the average a more intense magnetic field.

Provided the communication between "good and bad" curvature regions is adequate, average minimum-B should stabilize gross 
instabilities. Theory indeed indicates that at high temperatures, where plasma flow along lines of force is free and rapid, stability against gross modes should be achieved. Average minimum-B is another illustration of the important principle of picking a magnetic confinement geometry on the basis of its stability properties.

Promising though the minimum-B and average minimum-B principles are, it should be recognized that they are not panaceas. These principles are aimed mainly at eliminating grossly unstable behavior; their influence on the "fine grained," wave-particle, instabilities are much less marked. These instabilities must therefore be tackled by other means; for example, by control of the detailed physical conditions, or the size, or the shape of the plasma.

Many of the problems discussed thus far may be overcome if the Astron concept reaches fruition. The Astron configuration is expected to have two properties of significance: First, the plasma could have a distribution of particle velocities favorable to confinement. Second, the magnetic field intensity should increase outward from the plasma, thus preventing hydromagnetic instability. Such a configuration should eliminate end losses of the simple mirror, overcome the inherent hydromagnetic $i$ istability of the mirror, and provide a means of heating plasma at the same time.

\section{Technological Developments}

In the past the most important single factor setting the pace and defining the level of experimental research in controlled fusion 
has been in the area of technological development. These developments have encompassed an extremely broad range of techniques, including:

1. The storage, switching, and ultra-fast delivery of large quantities of electrical energy.

2. The generation of large volumes of intense, precisely shaped, magnetic field, both pulsed and steady-state.

3. The achievement of extremely high vacua in large chambers, and in the presence of intense particle beams.

4. The development of wholly new methods of generating intense directed bursts of high energy plasma.

5. The development of intense and precisely focussed beams of energetic charged particles or neutral atoms.

6. The extension of spectroscopic, microwave, laser beam, and electrical measurement techniques to cope with the special problems of "plasma diagnostics."

In some cases the special technological developments required prior to the construction and operation of an experiment have taken several years to complete. Each increase in the level of experimenta? sophistication has had to be accompanied by extensions of the technology.

Many of the techniques developed in fusion research have been taken over directly by other fields of modern research. As an example, plasma diagnostic methods, ultra high vacuum techniques and particle 
beam and plasma generation systems developed in fusion found immediate application in propulsion, simulation, and instrumentation needs of the space program.

Fortunately, at this stage in fusion research, some of the most difficult of the needed technological developments are completed, and the emphasis can accordingly be shifted more heavily toward the planning and execution of the experiments themselves. This is an extremely important change, one that can be expected to lead to a substantial increase in the rate of scientific output in many areas of fusion research.

Some of the developments that will contribute to this increase are in the field of cryogenics. Cryogenic techniques have at least two important applications in fusion research: (1) In the achievement of ultra-high vacuum by "cryo-pumping" (freezing out impurity atoms), and (2) In the generation of large volumes of intense magnetic field with no expenditure of electrical energy, by means of the new superconducting (zero electrical resistance) materials. This latter development seems extremely significant, not only for the short-range needs of fusion research, but for the long range promise of the whole concept of a fusion reactor, where magnetic field would be a critical commodity.

\section{J. "The Shape of Things to Come"}

In fusion research, as in many other fields, one result is worth a thousand predictions. Speculation as to the course of events 
is bound to be just that, speculation. Nevertheless, there are certain aspects of the directions in which the search for fusion power might proceed and the possible results that can be seen, at least in outline. Even the outlines excite the imagination. Here is a possible course that fusion might take: In the short range one would see the patient unraveling of critical aspects of the question of plasma instabilities.

Here, in addition to selective experiments intercomparisons between results found in ostensibly widely different circums:ances would be valuable. For example, a result found in an open-ended system might supply a key bit of information needed in the evaluation of a proposed closed system. At this phase it is essential to study a wide variety of systems, since the problem of plasma confinement and stability needs comprehensive study if a clear picture is to be obtained. In this period of rapidly developing plasma technology, the pace of research is determined by the time needed to prepare for an experiment; usually much less time is needed to obtain the scientific results. Thus, if the fusion physicist could, at will, wave a magic wand and create his confining magnetic field, in any shape desired, and then fill his confinement chamber with plasma of just the characteristics he desired, his research timetable would be very different: The salient features of the physics answer he is seeking could probably be exposed in a matter of months, from start to finish. 
During the exploratory phase of fusion research, the "bright idea" is an especially important element. Within the last year or so, "bright ideas" have appeared that have had a major effect on the course of research. An example is the idea of plasma stabilization by "average minimum-B," an idea that may be of profound importance to the future of toroidal confinement systems. It seems highly likely that more "bright ideas" will appear in due course. Their effect could be equally catalytic.

Behind the evolving story of confinement and stability the evolution of plasma technology will go on, with a most significant result: If adequately stable confinement of dense thermonuclear plasma can be demonstrated, the technical means for generating and confining these plasmas will already be well in hand, thus accomplishing a critical technical step on the way to fusion power. This same basic understanding and technology could be carried over into other practical or scientific applications of plasma technology, such as communications, national defense, space exploration, particle accelerator technology or astrophysics. Examples where this is already coming about include: ultra high frequency tubes, upper atmospheric weapons effects, plasma propulsion, new ideas for particle accelerators, and the theory of magnetic clouds.

If it is further assumed that the above exploration and consolidation phase of fusion research is successfully passed, the final phase 
of fusion research will probably be largelv shaped by economic or social-political factors that cannot be predicted with any certainty. This phase might see a rapid application of fusion research results to practical fusion power, stimulated either by intrinsic economic advantages of fusion or by considerations of safety or pollution vis-a-vis other energy sources. Just when fusion power might emerge as a major (or sole) power source cannot be predicted; that it will someday occur, given proof of scientific feasibility, there is virtually no doubt.

What might the fusion reactor of the future look like? It would be premature today to try to draw a blueprint for the fusion reactor of tomorrow. Yet some things are clear from what is already known today, so that the general features of what a "magnetic bottle" type of reactor would look like can be foreseen. Assuming first that stability, adequate to permit long-time confinement of plasma of intermediate density (as defined in Section G), is achieved, this regime could lead naturally to the idea of a large, continuously operating power plant, presumably with power output comparable to large present-day central power plants. The confining magnetic field would probably be provided by use of either superconducting or cryogenic magnets (normal conductors at low temperature). At least at first such a fusion reactor would doubtless use the deuterium-tritium ( $D-T$ ) reaction, with regeneration of the tritium through capture of the D-T reaction neutrons in a blanket surrounding 
the chamber. The constituent parts of the reactor per se would therefore be a large vacuum chamber, surrounded by the blanket and the magnet field coils and provided with auxiliary apparatus for evacuating the chamber and introducing and heating the fusion fuel. A high pressure steam turbine and generator would complete the system, except for equipment needed to regenerate and recirculate the tritium. Thus, the reactor itself would be in some ways similar to a modern steam plant, both as to size and function.

Suppose long-time stability is not in fact achievable? The pulsed reactor (Section $G$ ) then offers a possible alternative solution. Here a smaller, repetitively operated, system would be indicated. Such a system would have to face appreciably greater material and technological problems than would a steady-state system, but these problems do not at present appear insurmountable.

What is the probability that some wholly new approach to fusion power might be discovered that would bypass the need for magnetic confinement? Such a possibility cannot be definitely ruled out, but thirteen years of highly motivated, intensive study of the fusion problem have failed to turn up any workable alternative, though many proposed solutions have been advanced. That any solution would not involve plasma phenomena in some way seems highly un1ikely. 
For the even more distant future if fusion reactors can be perfected, there is an additional possibility for a revolutionary change in power generation. This is the promise of direct conversion of the fusion energy to electricity, particularly if the D-D reaction could be exploited. An understanding of plasma behavior will almost unquestionably permit the investigation of efficient and inexpensive means for direct conversion. What better environment for direct conversion could exist than the fusion reactor itself, with its controlled plasma and electromagnetic environment?

Today, one can only talk of "possibilities" in fusion power. Yet the avenue to finding the reality of these possibilities is open to us today, through pursuit of controlled fusion and high temperature plasma research.

K. "The Fusion Handbook"

The most significant aspect of the cutting edge of present day fusion research and its rate of progress toward the goal of controlled fusion does not lie in impressive technological developments nor even in the many milestones (relative to plasma temperatures, densities, or confinement times) that have been passed. It lies instead in the fact that experiments aimed at elucidating crucial aspects of the physics problem of plasma stability are being performed, and in the fact that these experiments are advancing hand-in-hand with well-founded and 
relevant theoretical calculations. The kind of solid understanding that is being built in this way will endure long after the specffic technical achievements are forgotten, and this understanding will constitute the "handbook" from which fusion reactors may someday be designed.

The process here described is characterized by two trends. First, experiments aided by the new technology are being aimed more and more toward elucidating crucial physics points, and less and less toward unselective techniques. Second, experiments aided by modern digital computer technology are being directed toward more and more "realistic" problems. The convergence of the two trends is obvious, and of great significance. In these trends lies the surest promise that fusion research will find the right answers to the right questions, and in the shortest possible time. 
STATUS OF RESEARCH ON PLASMA PHYSICS AND CONTROLLED FUSION

\section{A. Research in the United States}

The U.S. program of controlled fusion research began in 1951 and was conducted as a classified program at AEC 1aboratories until the time of the Second International Conference on the Peaceful Uses of Atomic Energy in Geneva, 1958. At the time of the Geneva Conference, the U.S., the United Kingdom, and the Soviet Union jointly declassified their respective programs. It was clear then that the U.S. was conducting the most comprehensive and Intensive of the world-wide programs.

American scientists developed a major part of fundamental plasma theory, with special emphasis on the stability questions of plasma confinement. U.S. theoretical research on gross (hydromagnetic) plasma properties was especially thorough, and the major predictions of these theories have since been experimentally verified.

An important aspect of the U.S. program has been the construction of powerful devices both to produce the required plasma and to provide an environment for the study of plasma confinement. Using one of these devices, U.S. scientists at Los Alamos achieved the world's first clear demonstration of a thermonuclear plasma in the laboratory. Shortly thereafter, scientists at Lawrence Radiation Laboratory also demonstrated the production of a thermonuclear plasma, using a different type of device. 
The U.S. controlled fusion program is conducted principally at

four major sites:

1. Lawrence Radiation Laboratory, (LRL)

Berkeley and Livermore, California

2. Los Alamos Scientific Laboratory, (LASL)

Los Alamos, New Mexico

3. Oak Ridge National Laboratory, (ORNL)

Oak Ridge, Tennessee

4. Plasma Physics Laboratory, (PPL)

Princeton University, New Jersey

Brief summaries of the programs of these laboratories are given below.

\section{Research in AEC Laboratories}

Lawrence Radiation Laboratory.

All elements of the LRL program are aimed at achieving steadystate or long pulse-time confinement of high temperature plasmas of intermediate density. The major experiments and related theoretical effort are therefore directed toward understanding and achieving those conditions that will avoid excessive plasma losses owing to instabilities.

Three major aspects of the problems of producing and containing a hot plasma are being pursued at the Lawrence Radiation Laboratory. These are:

*More detailed summaries of the Laboratory programs are contained in the Appendices. 
(a) Magnetic mirror experiments.

(b) The Astron experiment.

(c) Toroidal confinement experiments.

In addition, basic plasma and atomic physics research on topics relevant to controlled fusion are carried out.

The Magnetic Mirror Program consists of three mutually complementary experimental devices, operating in different plasma regimes, but each aimed at elucidating some aspect of the problem of stable confinement. These devices are: (1) Table Top, (2) $2 \mathrm{X}$, and (3) ALICE. A key feature of all three of these devices is the ability to employ some form of positive-gradient (minimum-B) field. In all cases the use of minimu-B fields has already proved effective in suppressing the gross flute instability by which the plasma drifts rapidly across the field lines and is 10st. The next task is the thorough study of wave-particle instabilities and ways to suppress them.

Table Top is a small, flexible facility in which a small volume of hot-electron plasma with an electron temperature of about $10 \mathrm{keV}$ and density of about $10^{11} / \mathrm{cm}^{3}$ is produced by plasma injection and magnetic compression. It has proved to be effective in the study of minimum-B stabilization. Recently, the nature of certain types of wave particle instabilities, which can only be observed properly when the flute instability has been eliminated, has been studied. 
The $2 \mathrm{X}$ is a relatively large machine in which energetic plasma is injected at one end along a guide field, trapped in a confining region and heated by magnetic compression. The resulting plasma has an ion temperature of about $5 \mathrm{keV}$ and density of about $10^{14} / \mathrm{cm}^{3}$, conditions which result in the production of substantial numbers of neutrons by fusion processes occurring in the deuterium plasma. These neutrons are very useful for following the timehistory of the p1asma.

Minimum-B stabilization of the plasma has eliminated the flute instability, and indications of plasma loss both by charge exchange due to contaminating gas emitted by the walls of the confinement chamber and by microinstabilities (wave-particle instabilities) have been observed. The $2 \mathrm{X}$ machine is one of the few experimental devices which produces a plasma with temperature and density close to those deemed necessary for a thermonuclear reactor, thus providing an opportunity to examine the problems of containment of a plasma with properties which close1y approach those of practical significance.

ALICE is a steady-state mirror machine in which the field strength is constant during times of interest. A beam of energetic (20 keV) neutral atoms traverses the confinement chamber in which the residual gas pressure is maintained at less than $10^{-9}$ millimeters of mercury. Some of the energetic atoms are ionized as they 
pass through the confinement chamber by the Lorentz (electric) forces produced by their motion in the strong magnetic field. These atoms are trapped (as energetic ions) to form the hot ion plasma. The density of the resulting plasma has generally been in the range of $10^{8}$ to $10^{9}$ ions $/ \mathrm{cm}^{3}$, depending upon the injection conditions and magnetic field shape. Elimination of the flute instability has been accomplished with a sequence of minimum-B stabilizing field geometries, under conditions such that some suppression of the loss arising from microinstabilities was also observed. In all cases however, the attainable plasma density shows evidence of being limited to a value determined by the field geometry and by the characteristics of the injection method. For the immediate future a new field coil, shaped like the seam on a baseball, and a vacuum chamber of improved design, are being installed. The resulting minimum-B field will provide a deep well for plasma confinement and ensure a more efficient conversion of the energetic neutral atoms to trapped ions. The combination of these effects should potentially yield an increase in plasma density by a factor of about 100. P1ans have been made to introduce a spread in energy in the injected beam. Theoretical expectations are that losses due to microinstabilities may be suppressed if the plasma particles are spread in energy and angle. Experiments to be carried out on Alice in the near future are aimed at testing these expectations. 
The Astron concept for the solution of the problems of production and confinement of a hot plasma differs significantly from other concepts. Electrons of several MeV energy are injected into a cylindrical chamber within which a nearly uniform magnetic field is produced by external coils. In a region between weak magnetic mirrors the electrons are trapped in helical orbits forming a cylindrical layer of rotating charges. The self-field of the E-layer is opposite that of the field produced by the coil, so that the magnetic field inside the E-layer is reduced more and more as the E-layer is built up; the direction of the field on the axis would be reversed if sufficient electrons were trapped. If these conditions can be attained the field configurations would then have two very desirable properties for plasma confinment: closed lines of force as in a toroid and an internally created minimum-B field. The present Astron facility consists of a linear induction accelerator which produces an electron beam of about 150 amperes at an energy of about $4 \mathrm{MeV}$ with pulse time of 0.3 microseconds and a long cylindrical vacuum chamber with field coils in which to test the build-up and stability of the E-layer. In experiments to date single pulses of electrons have been trapped with an efficiency of about 25 percent to form a weak E-1ayer. The E-layer once formed is free of both hydromagnetic 
and wave-particle instabilities. The observed lifetime is several millisecands. The most significant feature of the E-1ayer already produced is that enough electrons are trapped to create in combination with the external magnetic field an absolute minimum-B configuration, a necessary condition for hydromagnetically stable plasma confinement. Since no instabilities are detected to date, which would limit the E-1ayer IIfetime, it may be concluded that the build-up to higher levels of self-field is limited by the injection rate of the accelerator. By incresing the rate of injection to 360 pps, overlapping of electron pulses has been successfully demonstrated. Subsequent1y, the present Astron accelerator has been modified to provide a burst of pulses at a rate $1440 \mathrm{pps}$, to test the E-1ayer build-up and stability as a function of the accelerator pulse rate. Operation at the high injection rate is expected in the near future. As soon as the results of these experiments are sufficiently favorable in terms of E-layer build-up and stability, it is planned to proceed with the construction of a new $4 \mathrm{MeV}$ accelerator capable of injecting electrons at a rate of a million pulses per second. The increased electron output of the new accelerator will provide enough electrons to build-up the E-1ayer to the point of field reversal in less than one mil1isecond if not inhibited by an unexpected violent instability. If a fully developed E-layer is achieved, as is expected, an 
absolute minimum-B configuration with closed magnetic lines will then be created. This unique configuration should eliminate,a priori,a11 hydromagnetic and most of the wave-particle instabilities. The plasma will be generated from neutral gas and heated by the energetic E-layer electrons by Coulomb collision processes. This step will be a crucial test of the Astron concedt, for it will demonstrate whether the unique properties of the field configuration created by the E-layer will provide stable plasma confinement.

The immediate objective of toroidal-confinement research is to test the effectiveness of magnetic shear and average-minimum- $B$ stabilization. The principal experimental device used for studying toroidal confinement at LRL is the Levitron, which consists of a toroidal chamber with a heavy ring conductor suspended centrally within the chamber. External coils on the chamber produces a field around the torus, $\left(B_{z}\right.$ field), and a strong current induced in the ring conductor produces a field at right angles, with circular lines of force, so that the combined field consists of helical lines of force, the pitch angle of which varies with the radius; - i.e., the field has the property of shear. The objective has been to study the effectiveness of shear in stabilizing plasma confinement in toroidal geometry. 
The plasma in the Levitron may be heated either by induced electric current or by microwave power. It is found that in either case a hot plasma is formed but that plasma particles are lost by rapid diffusion across the field due to instabilities. These results imply that magnetic shear alone does not suffice to ensure stable confinement in toroidal systems.

The Levitron has been modified to permit the generation of a weak minimum-average-B field and the observation of the relative effectiveness of such a field combined with magnetic shear in stabilizing plasma confinement. Meanwhile, study of various toroidal field geometries is continuing in the search for the most promising minimum-average-B configurations for future experiments. Stability studies suggest that a combination of minimum-average-B and strong magnetic shear is most promising.

\section{Los Alamos Scientific Laboratory.}

Scientists at Los Alamos produced the first clear demonstration of the achievement of a dense reacting plasma at thermonuclear temperatures. This was accomplished with a fast magnetic compression device (theta-pinch) in 1959. The present program at Los Alamos continues to emphasize research on such highdensity, short-confinement experiments.

Interest in fast pulsed experiments at Los Alamos is motivated by two factors. First, the amount of thermonuclear energy 
released by a thermonuclear plasma depends on the density of the plasma and the length of time it is confined. Thus it is equally useful from an energy standpoint to have high density and short confinement, as it is to have low density and long confinement. While research in the other three AEC laboratories is aimed at long confinement, Los Alamos has emphasized achieving high density. In achieving high density, Los Alamos scientists have been quite successful. The latest model of their theta pinch device (Scylla IV) releases fusion power at a rate of 100 watts during each pulse.

The second factor which motivates Los Alamos research on fast systems is related to the problem of instabilities. Most plasma stability theory (excluding the theory of gross instabilities) has only been applicable to low density plasmas. Plasma behavior may be quite different in the high density regime. Thus, an intensive study of the stability of high density plasma is planned. Such a comprehensive study does not seem possible in the open-ended systems that have been used in the past. Loss of plasma from the ends is so rapid that many important plasma phenomena do not have time to develop. To overcome this problem, Los Alamos scientists are presently designing a closed theta pinch. 
Scylla IV, the only physically large LASL experiment, is the most recent and most powerful in a series of devices utilizing fast magnetic compression (theta pinch) to compress and heat a deuterium plasma to thermonuclear temperatures at high densities and high beta (see section II-G). The regime of high beta is not otherwise being pursued systematically in AEC laboratories and is the least understood theoretically. The product of plasma density and containment time (see section II-G and III-C) in Scylla IV is higher than it is in any other AEC device except for the Dense P1asma Focus Experiment.

Extensive experimental studies, particularly with laser interferometry, have shown no instabilities in the hot plasma which is, however, short-lived because of end losses in the present linear device. Because end losses rather than instabilities presently limit containment times, it appears desirable to extend this research to a closed theta pinch. Such a device, 1 abelled "Scy11ac," is presently in the advanced design phase at Los Alamos. Continuing studies on Scylla IV are emphasizing extensions of containment time via the difficult technique of power-crowbarring and experiments pertinent to Scyllac, such as curved sectors and "bumpy" magnetic fields. 
The Dense Plasma Focus experiment produces its plasma at the end of a specially designed hydromagnetic gun. The hot thermonuclear plasma is produced at extremely high density $\left(3 \times 10^{19}\right.$ particles $/ \mathrm{cm}^{3}$ ), resulting in a product of density containment time about ten times higher than that from the Scylla IV device. The volume, however, is extremely small- a few cubic-millimeters. In this device, for the first time, a mixture of deuterium and tritium has been used, further demonstrating that true thermonuclear heating was achieved. Although this device may not appear to be in the main path to a thermonuclear reactor, experiments are underway to investigate the high density properties of the plasma itself and to devise methods of increasing the $\mathrm{nt}$ product even more. The dense plasma focus is already of great interest as a high intensity pulsed neutron and $x$-ray source.

The Hydromagnetic Plasma Gun program at Los Alamos has had success in efficiently producing fast high temperature plasmas suitable for injection into other thermonuclear devices. Such injection is of particular importance for systems which are other wise tightly closed magnetically and which do not readily allow plasma heating in situ. This program has of necessity been largely empirical in approach because of the difficulties in understanding the methods of operation of the hydromagnetic guns. Present studies are devoted to scaling up the gun capabilities 
by a factor of ten with the hope eventually of being able to increase the yield by at least a factor of a hundred. Further theoretical studies of the acceleration mechanisms involved are urgently needed and being attempted. At the same time, detailed experiments on transverse and parallel injection of gun plasmas into external magnetic fields are underway.

Further programs pointed at increasing plasma densities by large factors are (a) Scyllacita: a specially designed, small theta pinch which is about ready for test with explosive compression of its magnetic field and plasma; and (b) Columba: an experiment to superimpose a linear pinch on a theta pinch.

Mathematical and Theoretical physics efforts are being heavily increased in several areas at LASL, including:

1) design of closed magnetic field configurations with desirable equilibrium and stability features;

2) high beta containment theory;

3) development of stability criteria for more realistic plasma mode1s than used previously;

4) studies of ballooning instabilities as applied especially to Scyllac.

5) studies of average minimum-B closed systems, as applied to Scyllac and the Caulked Cusp systems; and

6) computer numerical studies of rapid1y pulsed electric and magnetic fields. 
Oak Ridge National Laboratory.

The emphasis at the Oak Ridge National Laboratory has traditionally been upon research with plasmas in the steady state, as opposed to pulsed, experimentation. The result has led in several cases to unpredicted and surprising results which, even after the fact, are understood theoretically at most only in a general qualitative way. Steady-state operation has experimental appeal because the plasma can be gently manipulated into varying modes of performance.

Molecular Ion Injection - The DCX-1 Experiment. This classical Oak Ridge experiment has gone through three major phases, which are described in Appendix 3. A beam of molecular ions, accelerated to $600 \mathrm{kev}$, makes a loop in the magnetic field between two mirror coils, and by dissociation of some of the molecular ions in that region, a population of super-hot atomic ions is established.

The past accomplishments of DCX-1 include the establishment of fast ion densities of $2 \times 10^{9}$ per cubic centimeter, using carbon arc dissociation; the attainment of mean residence times of over two minutes at lower densities under conditions of high vacuum; and the recent eludication of $\mathrm{DCX}-1$ phenomena under a powerful trapping method called Lorentz dissociation. The latter work has shown that densities are limited to $2 \times 10^{8}$ per cubic centimeter when the mean residence times are 10 seconds or more, and that the 
density limitation is imposed by a specific type of wave-particle instability, called the "negative mass instability." To avoid the negative mass instability, the DCX-1 work must now be redirected toward infection into a minimum-B configuration --a direction that work in several other laboratories has shown to be desirable because of 1 ts suppression of other kinds of instabilities. Coincident theoretical work, and some trials on DCX-1, have shown that it is necessary to inject with a large spread in particle energy, and that it is desirable also to inject with a spread in angle.

Molecular Ion Injection - The DCX-2 Experiment. This is an experiment similar in nature to DCX-1, but arranged so that the molecular ions have a long spiral patk within the region between magnetic mirrors. When a lithium arc is used, nearly all of the injected molecular ions undergo dissociation and trapping. Densities of fast trapped ions are typically $5 \times 10^{9}$ per cubic $\mathrm{cm}$, which is the highest achieved anywhere in high-energy injection experiments.

A curious temperature upgrading effect, previously seen in $D C X-1$, is dramatically present in $\mathrm{DCX}-2$. Since the molecular ions are al1 injected at $600 \mathrm{kev}$, the atomic ions are a11 trapped at $300 \mathrm{kev}$. After a short time, the ions "shake down" to a more natural energy distribution, but in doing so some lose energy and 
some gain energy. Loss mechanisms quickly dispose of those which have lost energy, so when one looks in the steady state he sees mostly those that have gained energy. In an experiment, the average energy may be three or more times the energy at which trapping has taken place.

Electron Cyclotron Heating and the INTEREM Experiment. Although the "minimum- $B$ " configurations described in Section II-H of this document are promising and perhaps necessary, they are also expensive and power-consuming. If plasma physics can offer other means of providing stability, the cheapness of simpler magnetic systems would have strong appeal.

The electron cyclotron heating experiments at Oak Ridge have revealed a region of stable confinement at beta of $6 \%$ or more, for plasma established between simple mirror coils. The plasma in itself is not a fusion plasma, inasmuch as the ions are cold, but its stability opens the following channels of application:

1) To modify it toward fusion. This means heating the ions, and one experiment called INTEREM has been assembled to do this. Here neutral deuterium atoms at $20 \mathrm{kev}$ are injected into an electron cyclotron plasma, and are trapped by ionization. So far, fast ion densities of over $3 \times 10^{7}$ per cubic $\mathrm{cm}$ have been 
reached, with confinement times in the millisecond

region. A second objective of INTEREM is to reduce

the ambient pressure around the stable plasma; details

are given in Appendix 3.

2) For hydridization with molecular ion injection.

3) For application in various devices where a body of clean steady-state plasma is to be created in a localized region deep within a magnetic field. No guns, injection schemes, or rising magnetic fields are necessary.

4) To study instabilities. Although the electron cyclotron plasma can be held steady for hours if desired, it can also be deliberately tripped into instability.

Plasma Heating by Beam-Plasma Interaction. This work is directed toward heating rather than confinement. It is largely empirical, but the results have been unexpected and intriguing, leading into further channels of high experimental appeal.

The heating depends upon purely a plasma phenomenon, namely, efficient transfer of energy from an electron beam going along a magnetic field to the particles of a plasma into which it penetrates, and which are set in motion perpendicular to the magnetic field. Methods have been found of heating preferentially either the electrons or the ions. In ion heating (Burnout $\mathrm{V}$ experiment) the Oak Ridge work has produced a steady-state plasma of density $5 \times 10^{12}$ ions per cubic $\mathrm{cm}$ and temperature $1 \mathrm{kev}(10,000,000$ degrees) with a 
small proportion of the ions reaching energies of $170 \mathrm{kev}$. In another experiment, concentrating upon electron heating, a plasma she11 about four feet long and two feet in diameter has been established with an electron density of $10^{12}$ per cubic $\mathrm{cm}$. To assess the usefulness of this plasma for fusion, we must see how it performs in magnetic confinement regions, and bear in mind its possible combination with other means of establishing hot-ion plasma.

The foregoing Oak Ridge work is supplemented by theoretical studies that are both applied to the experiments and selfsufficient on their own. Engineering development, vacuum studies, atomic cross-section measurements, ion source development, plasma stabilization by wall treatment, sputtering measurements and studies of stabilization by average minimum-B add to the total effort.

Princeton Plasma Physics Laboratory.

Since its inception in 1951, the controlled fusion program at Princeton University has had as a broad goal the use of closed or toroidal magnetic fields for containment of a thermonuclear plasma. Provided that equilibrium and stability problems are solved, a toroidal system would have important advantages over open-ended systems in that end-1osses would be absent. Although the simple torus does not possess an equilibrium, a torus distorted 
into a figure-8 (in which the magnetic lines are twisted) should solve this problem. The effect was verified in the laboratory. Subsequently, a number of additional types of toroidal equilibrium have been demonstrated, including the system where, instead of using a figure-8, the magnetic lines are caused to twist by an additional external set of helical windings. This latter form should also be stable to some forms of hydromagnetic instability.

The general problem of stability is an extremely complicated one which requires a thorough scientific knowledge of the basic nature of plasma, knowledge which simply did not exist ten years ago. From the outset Princeton has had a strong emphasis on theory. Thus it became clear quite early that a broad program of basic scientific research was required in addition to the more empirically-oriented applied research, and since 1959 the effort has been divided about 50-50 along these 1ines.

Early experimental work demonstrated that a high level of technologocal sophistication was required in order to get firm results. For example, the loss of plasma tended to be masked by large amounts of gas released from the walls during the period of plasma containment. By the development of ultra-high vacuum systems and a new concept called a divertor, it was 
finally possible not only to avoid complete domination by impurities and the resultant masking of the basic processes taking place, but to reduce the role of impurities to a point where they are negligible. The Model C stellarator incorporating these improvements has made possible a detailed and extensive investigation of plasma confinement. This large device is in the form of a race track $1200 \mathrm{~cm}$. long with a discharge tube $20 \mathrm{~cm}$. in diameter surrounded by coils which produce magnetic fields of up to 50 kilogauss. Various types of helical windings may be employed as well as a variety of heating methods.

Over a period of years it has been possible to demonstrate that the plasma confinement time in a stellarator of this type is proportional to the cross sectional area of the tube, to the magnetic field strength, and inversely proportional to the temperature. This time is considerably shorter than that expected in the absence of instabilities. Indeed, under reactor conditions assuming present loss rates a tube 150 feet in diameter would be required. This is far too large to be practical. Considerable effort has been and will continue to be made on understanding the reasons for this high loss rate.

The principal method of heating used in the early work was Ohmic heating, effected simply by passing a current through the 
Initially neutral gas. According to both theory and experiment, this method is useful for creating a plasma from the cold gas and heating it to about a million degrees. Furthermore, it was found, provided that the current is kept below a certain critical level, that present loss rates are not enhanced by this current. Electron cyclotron and ion cyclotron heating methods are also being employed. Considerable theoretical and developmental work has also been done on another heating method called "magnetic pumping." This is a slow method of heating requiring large equipment which should become effective if and when the present loss rates have been substantially reduced. There is the important compensating advantage, however, in that this slow heating process should have much less tendency to produce microinstabilities.

Recent theoretical work has indicated that two different forms of instability should be expected in a system such as the Model C stellarator. One called the "resistive $\mathrm{g}$ mode" is a form of the flute instability which enters when the finite resistivity of the plasma is taken into account. The other, the "drift" or "universal" mode is due simply to the drop in pressure that must take place between the hot plasma and the walls. It appears possible to stabilize both these modes by 
proper shaping of the magnetic field. The first requires that the average magnetic field strength increase toward the wall; the second requires shear in the magnetic field. A variety of suggestions have been made both in the U.S. and abroad on ways of accomplishing these ends. Evaluations of the relative merits of these systems requires not only detalled calculations, but also refinements of the existing theorles. Both these processes are now under way and are expected to culminate soon in a proposal for a new device, and/or a major modification of the Model C Stellarator.

\section{Research at Universities}

High temperature plasma physics was born in a classified program at AEC laboratories. Since 1958, when the AEC program was declassified, university contributions to this field of physics have risen sharply.

Plasma research requires the talents of mathematicians, physicists, and engineers. It demands strong interdisciplinary programs. Faculty and students from several university departments can develop extremely stimulating and fruitful collaborations. For these and other reasons, plasma physics is well suited to play a vigorous role in university research. 
University interest in plasma physics is not simply, nor even principally, a result of interest in controlled fusion. With the growth of space exploration, interest in astrophysical plasmas has soared. Astrophysical plasma research is thriving on many campuses, drawing heavily on techniques developed in the thermonuclear program.

Engineering departments in universities are attracted to plasma physics by the promise of many practical applications. For example, engineers are developing magnetohydrodynamic (MHD) generators, which burn conventional fuels to produce plasmas whose thermal energy is converted directly into electrical energy. The design of plasma propulsion engines for space vehicles is also an area of interest to engineering departments.

For many years the technological problems of producing and studying plasmas hampered the performance of basic experiments. As a result of the intensive research toward controlled fusion, most of these difficulties have been overcome, so that university physics departments are now expanding their role in basic plasma research.

The large size of most thermonuclear devices has inhibited the development of controlled fusion research at universities. 
University scientists have, however, made many contributions not only in the ffeld of basic plasma physics but also in some of the more programmatic aspects of the CTR effort. This section describes several specific university programs supported by the AEC's thermonuclear program.

At the University of California at San Diego (UCSD) outstanding research has been performed on most of the pressing questions of thermonuclear plasma stability. Sclentists there are in close liaison with the efforts at General Atomics and at LRL, and have recently participated actively in the International School of Theoretical Plasma Physics at Trieste, Italy. This school operates under the auspices of the International Atomic Energy Agency (IAEA).

Mathematicians at the Courant Institute of New York University are studying the basic structure of the plasma equations. This group predicted the conditions for gross (hydromagnetic) stability produced by minimum-B fields several years prior to the experimental demonstration of this effect. They have studied the theory of magnetic mirror containment and are acknowledged experts in the field of shock wave phenomena in plasmas. 
University of Tennessee physicists continue to play a leading role in the development of wave-particle (microinstability) theory. Their calculations on electrostatic microinstabilities have been applied successfully to the DCX-1 experiments at ORNL, and have helped to stimulate experiments on "beam-plasma" interactions at several institutions.

Many university plasma programs have developed from residence of university professors at AEC laboratories. For example, close ties exist between the Nuclear Engineering Department at the University of Michigan and the AEC's program at LRL.

University programs not directly related to thermonuclear plasma studies have played a strong auxiliary role. Examples include: a program of atomic collision measurement at the Georgia Institute of Technology; the codification, at Swarthmore College, of techniques for magnetic field design; and the writing, at Yale University, of a critical review of plasma kinetic theory. In addition, a strong interdepartmental program in plasma physics has been thriving at the Massachusetts Institute of Technology and at Stanford University for many years.

A few AEC-supported programs in universities have research efforts which are "thermonuclear" in their own right. At the University of Wisconsin a hydromagnetically stable toroid, 
called an octopole, is filled with high temperature plasma from a plasma gun. Seven senior scientists and eighteen graduate students are performing experiments with this device. Another device which produces hot plasma for confinement studies is in operation at the Stevens Institute of Technology.

Research is not the only product of a university program. As thermonuclear devices become more sophisticated, 1arger experimental groups are required to utilize the devices effectively. In other areas of plasma applications, the demand for persons trained in plasma science and engineering is increasing. Strong university programs provide the training ground for needed talent.

\section{P1 asma Research Programs of Other Government Agencies}

Research on plasma is an important element in the programs of other government agencies a1so. University programs are of prime concern to the National Science Foundation. Space plasmas, plasma propulsion, and the plasma effects of space vehicle reentry are important to the National Aeronautics and Space Administration. Gaseous electronics, MHD generators, atmospheric research, plasma propulsion, and the analysis of weapons effects play an important role at the Defense Department. 
This report does not describe the achievements of these other agency programs, with three exceptions: one at the U.S. Naval Research Laboratory; Washington, D.C., another an NSF-supported program at the Massachusetts Institute of Technology (MIT), and the third a recently instituted Air Force program at Aerojet-General Division of General Dynamics Corporation.

The thermonuclear plasma research effort at the Naval Research Laboratory has been especially useful to the AEC. Navy scientists have contributed extensively to the understanding of high density, collision-dominated plasma behavior by studying fast magnetic compression plasmas. They have emphasized spectroscopic study of plasma conditions, have evaluated the importance of field-mixing as a heating mechanism, and have studied behavior of plasma in the presence of multipole fields.

In the MIT Nuclear Engineering Department, several computer studies have been made of conceptual thermonuclear reactors. The calculations considered neutron economy and tritium regeneration in moderating blankets, as well as other quantities of engineering importance, such as choice of coolant. The results of these calculations give added strength to the proposition that thermonuclear reactors $c$ an be built if adequate plasma confinement can be achieved.

At Aerojet-General, motivated by possible future applications to space propulsion, an Air Force-supported research program has recently begun on fusion plasma containment. A large minimum-B magnetic bottle has been constructed and attempts will be made to fill this container with energetic plasma by injection of neutral hydrogen atoms. 


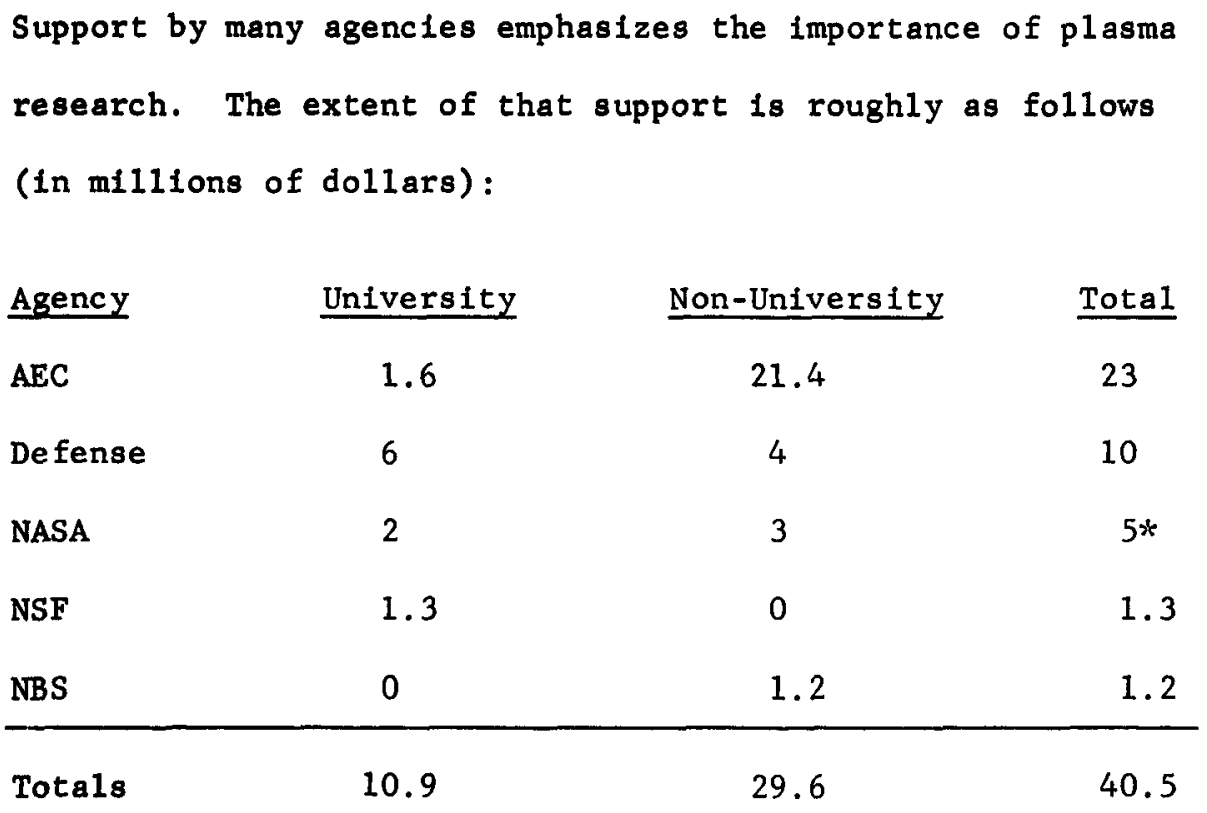

\section{Plasma Research By Industry}

Partly because of the newness of plasma science and partly because of the long-range nature of some plasma applications, most plasma research has been performed in government laboratories or in universities. In certain areas, however, industry contributions have been substantial. For example, strong government-supported activities in applications like MHD and plasma propulsion are being carried out by industrial laboratories.

*The NASA total does not include amounts devoted to developmental plasma devices for space propulsion, or to s pace plasma experiments. 
Privately supported industrial activities in controlled thermonuclear research are in progress at General Atomics Division of General Dynamics Corporation and at the General Electric Research Laboratory. A program with partial AEC support exists at United Aircraft Corporation Research

Laboratories and at Westinghouse Research Laboratories.

At General Atomics a wel1-supported research program, financed jointly with the Texas Atomic Energy Research Foundation, began in 1957. Early experiments were concerned with the linear pinch and the toroidal pinch. More recently, emphasis has shifted to devices using plasma guns for injection into mirror and cusp magnetic fields. The latter work led to operation of linear and toroidal devices with octopole magnetic fields.

General Atomics scientists have developed plasma guns and magnetic guide fields for reducing their impurity content. They have demonstrated that flute instabilities in a mirror machine can be stabilized by cold plasma. The theoretical contributions of $\mathrm{GA}$ scientists have been impressive.

The General Electric Research Laboratory also initiated a program in high temperature plasma physics in 1957. GE scientists have performed theoretical work in several areas of plasma physics. In 
their experimental work they have emphasized research on theta pinch plasmas, a working model of which was shown at the New York World's Fair. Measurements on the plasma produced by this device have included electron temperature by spectroscopic observation of impurities. The fon energy spectrum was determined by an ingenious method, in which the rate of fusion in deuterium alone was compared with that in mixtures of deuterium and helium-3. Recent1y, the GE scientists measured the energy spectrum of ions escaping from the ends of the theta pinch, and investigated the equilibrium of the plasma in a curved magnetic field.

Scientists at the United Aircraft Corporation Research Laboratories in Hartford, Connecticut are performing work that is supported in part by $\mathrm{AEC}$ funds. They are investigating an interesting new method for producing a high temperature plasma. A lithium-hydride pellet is electrostatically suspended in vacuum in the center of a magnetic bottle and then exposed to the focussed beam of a high-powered ruby laser. Fully-ionized, high-temperature, high-density plasmas have been formed in this manner, and these plasmas are now being studied in a variety of open-ended magnetic fields. A related program is underway at Westinghouse in Pittsburgh.

\section{B. Foreign Programs}

The most striking aspects of foreign programs in controlled thermonuclear research have been their recent growth and the 
extent of the national commitments involved. In 1958, the U.S. effort (who1ly AEC supported) was 1arger than that of any other country. Since then, the total world manpower has almost doubled, while that of the USAEC has remained almost constant. It follows that the percentage increase in most of the foreign programs has been very large.

It would seem that foreign governments have recognized the considerable present value of thermonuclear research and the great future potential of high temperature plasma research. The national commitment of the U.K. (in terms of the fraction of their total research effort) is impressively large. In the USSR, policy statements were made by Premier Khruschev to the Twenty-First Congress of the Cormunist Party (November 1958) assigning fusion research a very high national priority. The significance of these statements is demonstrated by the rapid expansion of Soviet fusion research in the years that followed and by the development of an extremely broad-based, diversified program.

USSR.

In manpower devoted to controlled thermonuclear research the Soviet Union leads the world. Their effort is twice the U.S. 
effort. In plasma theory the Soviets are preeminent and at this time their effort on theory is about four times the U.S. effort. In number and variety of major experimental devices the Soviets also lead the world.

Soviet controlled fusion research is conducted at seven national 1aboratories :

1. A.F. Ioffe Physico-Technical Institute of the Academy of Sciences of the U.S.S.R., Leningrad.

2. D.V. Efremov Scientific Research Institute of Electrophysical Apparatus, Leningrad.

3. Physico-Technical Institute of the Academy of Sciences of the Georgian S.S.R., Sukhumi.

4. Physico-Technical Institute of the Academy of Sciences of the Ukrainian S.S.R., Kharkov.

5. I. V. Kurchatov Institute of Atomic Energy, Moscow.

6. Institute of Nuclear Physics, Novosibirsk, Siberia

7. Lebedev Physics Institute, Moscow.

A major field of Soviet theoretical effort is nonlinear kinetic theory. This field is in a state of relative infancy but many scientists believe that nonlinear theory will eventually permit the quantitative prediction of plasma losses due to microinstabilities. This is a key question in fusion research today. 
An example of the high quality of Soviet research is that Soviet scientists made the first demonstration of the suppression of gross (hydromagnetic) instabilities by the use of minimum-B fields. Since then the value of minimum- $B$ has been verified in several countries. This type of field has been incorporated in many experiments, both existing and under design. The success of this one experiment in the USSR thus has had a major impact on the course of world fusion research.

Another example of the high quality of Soviet research is found at the new Soviet laboratory in Novosibirsk, where a particularly fruitful interplay between theoreticians and experimentalists has resulted in a novel experimental device. A "magnetic piston" is formed by rapidly increasing a magnetic field. Convergent cylindrical shock waves are produced, thus heating the plasma. This method is called "turbulent heating." It differs from fast magnetic compression experiments (theta pinches) principally because the plasma density is about 100 times smaller in the turbulent heating case.

United Kingdom.

The British have created a large national center for controlled fusion research at Culham, near Oxford. An impressive variety of experimental facilities exists, including neutral injection 
Into a Minimum-B mirror, fast magnetic compression, gun injection and trapping, and a variety of pinch and cusp devices. Recently a "turbulent heating" experiment was initiated. A notable feature of the Culham research program is the very extensive advanced engineering and development support that is provided.

The scientific and engineering manpower at this one controlled fusion center is more than two-thirds of the total U.S. manpower at AEC laboratories. In population and gross national product, the United Kingdom is a much smaller country than the United States. Thus the size of its commitment to controlled fusion research is impressive. Approximately $25 \%$ of the Culham staff are scientists from other countries on short term (usually one year) appointments. The laboratory management has instituted the short term appointment system to encourage a steady influx of new ideas and to ensure meaningful interactions with foreign programs.

A major element of the British experimental program is the neutral injection experiment called "Phoenix." It is similar in principle to the ALICE Facility at LRL, Livermore. When Minimum-B magnetic fields were activated, hydromagnetic instability was suppressed. The plasma density builds up as a greater current of particles is injected, in accordance with simple theory. The density which can be achieved in this way 
should eventually be limited by microinstability losses but, so far, such a limitation has not been observed.

\section{West Germany.}

A strong controlled thermonuclear research program is in progress in West Germany. At the Max Planck Institute near Munich, the Germans have constructed a large complex for fusion research. The manpower at this site is approximately half the total manpower at AEC national laboratories. Sclentists at Max Planck have strong basic programs on a variety of confinement schemes. Graduate students from the University of Munich are heavily involved on all experiments. Recently a powerful new theta-pinch experiment for studying high density thermonuclear plasma was completed.

At the International Conference on Controlled Fusion, held September 6-10, 1965 at Culham, Max Planck scientists discussed two especially interesting experiments using low temperature alkali plasmas in toroidal magnetic geometry. Theoretical treatment of low temperature alkali plasmas can be performed more rigorously than for high temperature hydrogen plasmas. For this reason, many correlations between theory and experiment can be made.

A smaller center for fusion research exists at Julich, Germany, The program there consists of intensive study of theta pinch plasma confinement. 


\section{France.}

In France, controlled fusion research is carried out mainly as an activity of the ultra-modern, multi-purpose nuclear research center at Fontenay-auz-Roses. A large variety of experiments are being performed and the program is well-supported financially. As in the case of some of the other European programs, partial financial support is provided by Euratom.

French scientists at Fontenay have successfully suppressed the flute instability in a magnetic compression experiment by Minimum- $B$ methods. In the French experiment the plasma density was a thousand times greater than the plasma density of the first successful USSR experiments on Minimum-B stabilization.

A smaller group active at Saclay has specialized in studies of the interaction of radio-frequency waves with plasma - a field which has been dormant for several years in the U.S.

Japan.

The most dramatic of the recent foreign expansions in controlled fusion research has occurred in Japan. In men, machines, and publications the Japanese program is making many solid contributions to world fusion research. Most of the Japanese effort is centered at Nagoya University, but scientists are also active at other locations, such as Osaka University. Several Japanese physicists have aided the AEC's program at Princeton. 
A major controlled fusion device at Nagoya uses radio-frequency

heating followed by adiabatic compression. The Japanese

scientists state that it is important to prepare a high temperature

plasma as quiescent as possible before it is compressed by a

magnetic field. Their research is directed toward this end.

This is the first year of operation for the new device.

\section{Other Nations.}

Several other nations have active programs in controlled fusion research. The largest of these are in Italy, Sweden, and the Netherlands. The combined manpower of these three nations is equal to about one-third the U.S. effort at AEC national laboratories. A small program also exists in Australia.

International Cooperation.

Controlled fusion research, being a difficult field requiring extensive development of the basic science, profits greatly from international conferences and personne1 exchanges. There have been three major international conferences: Geneva, 1958; Salzburg, 1961; and Culham, 1965. There have also been many smaller topical conferences and two international conferences of controlled fusion engineers.

The exchange of personnel has been more difficult to arrange than conferences. Controlled fusion research was classified 
secret until 1958. Much of the U.S. research was, and still is, performed at laboratories in close proximity to classified research. Except in special instances long term assignments for foreign scientists in the U.S. have been possible only at the Princeton and Berkeley sites.

The U.S. has engaged in a formal personnel exchange program with the United Kingdom in which approximately 8 scientists have been exchanged in each direction.

Personnel exchanges with Soviet scientists were accomplished recently on a limited scale. Two U.S. scientists, one from Princeton, and one from LRL, Berkeley, have gone to the USSR for 3 and 6 month work assignments at Russian laboratories. Two Soviet scientists are expected in exchange.

\section{SUMMARY OF PROGRESS}

The foregoing sections have provided a brief review of the present world effort in fusion. It is apparent to those that have been involved in this research that, taken worldwide, controlled fusion and high temperature plasma research have developed at a remarkable pace over the last four to six years, sparked primarily by the early U.S. work in this field. By now fusion plasma research comprises a well-established and viable area of scientific research. Since it is concerned with 
a whole new field of physics research, rather than with a specific technical development, it is not easy to compare progress in high temperature plasma and fusion physics with, say, the development of the atomic bomb, over like periods of time. A better comparison would be with the many years of theoretical and experimental work of a fundamental nature that finally led to the development of the bomb.

It would not be possible adequately to describe, nor even to list, in this summary all of the many significant developments that have occurred in fusion research since it first began, though some of these have been mentioned in Section II or in earlier parts of this Section. It is possible, however, to impart something of the solid nature of the progress in fusion by singling out some of the more significant steps of a theoretical or experimental-technical nature that have special significance for the understanding and control of high temperature plasmas. These steps provide the scientific and technical undergirding of present-day fusion research. They are listed without any attempt at precise chronology or origin.

They are:

(1) Development of the basic concepts of magnetic confinement; rotational transform and adiabatic confinement. (Established the basis for stellarator and magnetic mirror systems.) 
(2) Introduction of microwave, spectroscopic and magnetic and particle beam probe methods of "plasma diagnostics."

(The problem of finding out what a plasma is doing was once one of the most difficult tasks of fusion research.)

(3) Establishment of the foundation of the "energy principle" of instability theory, and its experimental confirmation. (This theory enabled the prediction of grossly unstable situations, and pointed the way to shear stabilization and, eventually, to the minimum- $B$ and average minimum- $B$ ideas.)

(4) Development of plasma and high energy particle injection methods for creating hot plasma. (Plasma obviously cannot be studied unless means for its generation are available; these two methods are among the most important.)

(5) Development of Plasma Heating Methods; Magnetic Compression; Radio Frequency. (P1asmas created in situ must be heated. These methods have been employed to achieve thermonuclear temperatures.)

(6) Application of the "collisionless Boltzmann" (V1asov) equation to the analysis of wave-particle and other instabilities in high temperature plasmas, and its experimental verification. (The whole modern theory of wave-particle instabilities depends on the validity of this equation.) 
(7) Development of ultrahigh-vacuum techniques for large-scale systems. (Long-time confinement requires extreme purity and very high vacuum.)

(8) Stabilization of hydromagnetic instabilities by minimum-B fields. (These experiments have had a major influence on fusion research.)

(9) Development of the technology of high magnetic fields, including pulsed and superconducting magnet coils. (No significant confinement experiment can be performed without the use of magnetic field technology; the future impact of superconducting coils on many areas of fusion research is expected to be very great.)

(10) Theoretical prediction of "average minimum-B" configurations. (These configurations are the most promising ones known for stabilization of toroidal systems.)

(11) Development of "non-linear theory" as applied to plasmas. (This theoretical development offers the promise of prediction of the effects of plasma instabilities.)

In parallel with the above scientific-technical milestones in fusion research there has occurred a steady upward march in the attainable plasma properties (temperature, density, purity, confinement time) that also represents major progress. Only a few years ago the achievement of a pure, well-randomized plasma at thermonuclear 
temperatures seemed far in the future. Today, the production of such plasmas is routine in many experiments. Although the triple criteria of (1) a dense plasma, (2) thermonuclear temperatures and (3) long-time plasma confinement has not yet been achieved, pair-wise combination of the above requirements have been. The accompanying Table summarizes representative values of these plasma parameters as achieved in the classes of experiments listed. Taken alone, these numbers would not portray a meaningful picture of fusion progress, but they do indicate the broad range of plasma conditions that have been achieved in high temperature plasma experiments. 


\begin{tabular}{|c|c|c|c|c|}
\hline Sys tem & $\begin{array}{l}\text { Density, } n \\
\text { ion } / \mathrm{cm}^{3}\end{array}$ & $\begin{array}{l}\text { Mean } \\
\text { Ion } \\
\text { Energies* } \\
\text { KeV }\end{array}$ & $\begin{array}{l}\text { Time, } t \\
\text { ms***x }\end{array}$ & nt** \\
\hline $\begin{array}{l}\text { Neutral Injection } \\
\text { Min-B Mirror } \\
\text { Machines }\end{array}$ & $10^{9}$ & 20 & 100 & $10^{8}$ \\
\hline $\begin{array}{l}\text { Molecular } \\
\text { Ion } \\
\text { Injection } \\
\text { Mirror Machine }\end{array}$ & $10^{10}$ & 1000 & 50 & $5 \times 10^{8}$ \\
\hline $\begin{array}{l}\text { Stellarators- } \\
\text { Resonant Heating }\end{array}$ & $10^{13}$ & 0.2 & 1 & $10^{10}$ \\
\hline Plasma Injection & $10^{14}$ & 5 & 0.2 & $2 \times 10^{10}$ \\
\hline $\begin{array}{l}\text { Theta Pinches } \\
\text { (Fast } \\
\text { Magnetic } \\
\text { Compression) }\end{array}$ & $10^{16}$ & $2-5$ & .005 & $10^{11}$ \\
\hline
\end{tabular}

*If the plasma is well-randomized, it is appropriate to define a "temperature" of the plasma. A rough conversion between mean ion energies in $\mathrm{KeV}$ and temperature is that $1 \mathrm{KeV}=10,000$ degrees centigrade. ( $\mathrm{KeV}=$ kilo-electron-volts)

** In the long range, "nt" values must exceed the "Lawson Criteria", typically about $3 \times 10^{14}$. In the short range, nt is not a reliable index of the research, except for its use to observe trends with the passage of time.

*** $1 \mathrm{~ms}$ (millisecond) $=1 / 1000 \mathrm{Sec}$. 
There have been two extensive reviews of the Controlled Thermonuclear Research effort in recent years: one by a subcommittee of the General Advisory Comittee in 1962, and another by a special Review Panel in 1965. In addition, the whole of plasma science has been surveyed by a subpanel of the Pake Committee of the National Academy of Sciences and by a panel contributing to the Energy Resources Report to be issued by the Office of Science and Technology.

\title{
The 1962 Report of the General Advisory Committee
}

This report was prepared by a subcommittee of the GAC, whose members were:

\author{
Dr. Philip H. Abelson, Chairman \\ Professor Manson Benedict \\ Dr. Robert A. Charpie \\ Professor Norman F. Ramsey \\ Professor John H. Williams
}

\begin{abstract}
Both positive and negative reactions to individual subprograms were recorded, but the general recommendation was "to continue to support Project Sherwood vigorous1y."
\end{abstract}


In support of this recommendation the subcommittee made the following statement:

"In the Sherwood program there remain many unresolved technical questions that can be decisive to the feasibility of controlled fusion as a source of economic power. These relate particularly to the problems of stable confinement of hot plasmas in configurations suitable for power, to methods of initially heating the plasmas, to problems of purity, etc. However, it appears that, if these feasibility problems can be overcome, controlled fusion power could be economically competitive at least with breeder reactors. For this reason we believe that the AEC should continue a vigorous program in controlled fusion research and in basic research in hot plasmas. However, this recommendation should be reviewed periodically since its continuing validity is dependent upon future technical developments."

The conclusions and recommendations of this GAC report are attached as Appendix I. 


\section{Review Panel}

This panel was appointed by the General Manager with the concurrence of the Commission. The membership was as follows:

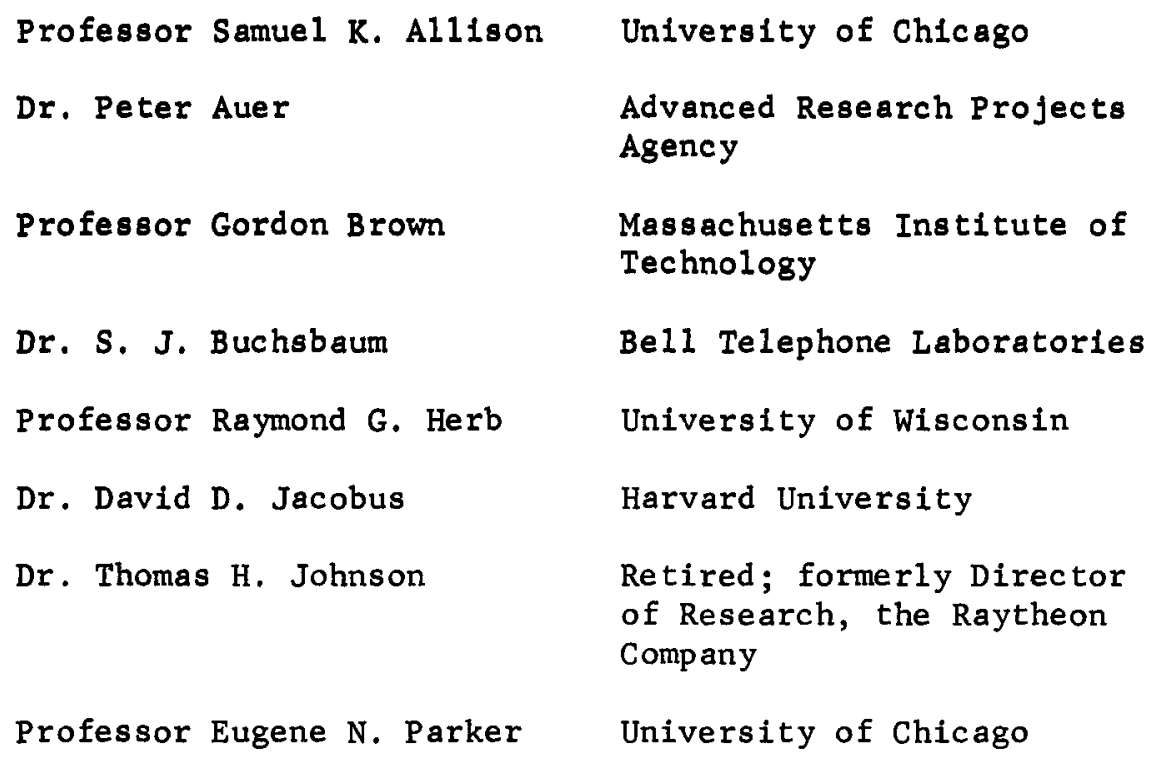

The first Chairman was Professor Allison. After his untimely death in September, 1965, Professor Raymond G. Herb became Chairman. Special measures were adopted to insure that the Panel could obtain a comprehensive view of fusion power research here and abroad:

1. The four major Sherwood laboratories prepared status reports designed particularly to meet the needs of the Pane1. These documents were discussed with the other 1aboratory directors and the $\mathrm{AEC}$, modified and then presented to the Panel at the time of its organization. Abbreviated and updated versions of the Laboratory status reports are presented in Appendix 3. 
2. In June and July, 1965, the Panel visited the four major laboratories. At each of the sites, scientists made presentations to the Panel.

3. Subgroups of the Panel visited several of the smaller Sherwood sites, and representatives from others were heard.

4. Presentations were also made to the Panel by representatives of Aerojet-General Nucleonics, General Atomic, Genera1 Electric, and the Naval Research Laboratory. In addition, discussions were held with Dr. Stirling A. Colgate and Dr. Henry D. Smyth.

5. Three members of the Panel went to the International Conference on Plasma Physics and Controlled Thermonuclear Research at Culham, England, in September, 1965.

6. Supplementing the above contact with scientists in the field, the Panel also had frequent discussions with those at $\mathrm{AEC}$ headquarters responsible for the administration of the Sherwood program.

The report of this Panel is presented as Appendix 2. It should be noted that the findings and recommendations of this report were made unanimously by the Panel members. 
This report was prepared as input to a general survey on the status and needs of physics research as a whole. The members of the subpanel on plasma physics were:

Professor Marshall Rosenbluth, Chairman

Professor William E. Drummond

Professor Melvin B. Gottlieb

Dr. Arthur Kantrowitz

Dr. Richard F. Post

Dr. Eli Reshotko

Professor Peter A. Sturrock
University of California at San Diego and Genera1 Atomic Corporation

University of Texas

Princeton University

AVCO-Everett

Lawrence Radiation Laboratory

NASA Lewis Research Center and Case Institute of Technology

Stanford University

Surveys were made of several areas involved in plasma research. astrophysics, gaseous electronics, propulsion, direct conversion, fusion, solid state plasmas, military plasma research, general plasma research.

With respect to the controlled thermonuclear research, the Panel concluded:

The fusion program is now showing great promise, with increasing agreement between theory and experiment in the critical stability area, and greatly improved experimental capabilities. A 1964 Energy Resources Study of the Office of Science and Technology recommended an approximate doubling of the fusion program within the next 5 years (up to the 1965 estimated level of the Soviet effort). We agree that fusion is a desirable national scientific objective and recommend that the following guidelines be used in this expansion: 
(a) It should be kept in mind that fusion is a long-range program and it should be planned as such. In deciding wh1ch large-scale experiments should be performed an important criterion should be the possibility of detailed comparison between theory and experiment so that an orderly bulldup of knowledge may proceed. Such knowledge will also serve other areas of plasma physics. The short-range objective at this time should be the attalnment of a quiescent plasma with physical parameters in the approximate thermonuclear range with which such comparisons $c$ an be made. On the basis of recent theoretical advances it appears clear that the minimum-B concept best meets these criteria at this time.

(b) Smal1-scale basic experiments such as beam-plasma interactions and Cs plasmas, which give a quantitative check on plasma theory in somewhat different physical regimes, are a very necessary component of the fusion program.

(c) A strong effort must be made to produce a flow of new people and ideas into the program. The invigorating effect of young people on the U.S.S.R. program greatly impressed the recent fusion exchange team. At the same time, scientists with fusion experience would provide valuable new viewpoints in other areas.

(d) It is recommended that support for fusion be diversified and not kept completely within a single agency.

We feel that these aims should be kept in mind even if it should prove impossible to obtain the desired increase in the overall program. 
SECTION $V$. EVALUATION OF THE OVERALL EFFORT ON CONTROLLED FUSION

In the period since the submission of the Review Panel's report, a number of meetings have taken place between representatives of the Commission, Directors of the major Sherwood projects, and members of the Panel itself. These meetings have permitted extensive discussions not only of the findings and recommendations of the Panel, but of additional topics relating to the progress of the Sherwood effort. In addition the Report of the Review Panel, together with a draft of the present policy and action document, was examined by a subpanel of the President's Science Advisory Committee (PSAC), by PSAC itself, and by the Commission's General Advisory Committee (GAC). Statements by the latter groups are presented as Appendix 6.

The overall evaluation of this program by the Commission is summarized in the paragraphs which follow. As will be seen, the Commission concurs in large measure (but by no means completely) with the findings of the Review Panel. Based on its evaluation, the Commission presents, in Section VI.A., the main features of the policy which, ir its view, should guide the planning of the AEC-supported program in plasma science and nuclear fusion research in the United States. Specific plans for implementing this policy are then outlined in Section VI.B.

\section{A. EVALUATION OF THE IMPORTANCE OF THE PROGRAM}

By any standard whatsoever, controlled thermonuclear research must be counted as one of the most challenging and potentially important efforts 
in the history of mankind. The original programmatic interest in this work - and still one of the strongest motivations for pursuing it is the hope of producing useful power from controlled thermonuclear reactions.

Clearly, it is far too early to sketch with any certainty the details of a full-scale fusion reactor. If this program is successful, however, such a reactor could be expected to have a number of general features of potentially great interest:

1) The fuel is readily obtainable. Indeed, for the ultimate case of a reactor burning deuterium alone (rather than a deuteriumtritium mixture) the fuel supply is not only easily obtained from water but is virtually inexhaustible as well.

2) Since the actual amount of fuel within the reactor is exceedingly small, and since the sudden addition of excess fuel would merely quench the fusion reactions, there would be no danger of causing a reactor "runaway" condition.

3) There exists the long-term possibility of conversion from plasma energy directly to electricity.

4) The final fusion reaction products (helium and neutrons) are non-radioactive. In a simple system, as presently conceived, the only radioactivity associated with the plant itself would be that which is induced by neutron bombardment of the construction materials. (For the case of a reactor using only deuterium as fuel, the tritium produced would be immediately consumed in the reaction chamber.)

In addition to the above programmatic interest in plasma physics and controlled fusion research, there are also compelling interests of a more basic nature for studying the behavior of plasmas. More than 99\% of the matter in the entire universe (as we know it) is in the form of highly ionized plasmas. The sun and stars are composed of

$$
\mathrm{V}-2
$$


it; the earth and other planets are bathed in it; plasma fills the vast regions of interstellar space. Its properties have received little attention until now. Yet its bearing on other fields of knowledge and on the future activities of mankind may well be profound.

In view of the above vast potentialities - both of long-range fusion power and of the importance of high-temperature plasma research this nation must maintain itself at the forefront of the research effort in this field.

\section{B. EVALUATION OF THE PRESENT STATUS OF THE U.S. EFFORT}

Sections II and III of this report review the basic problems of the field of controlled fusion and summarize the work underway both in the United States and throughout the world. The evaluation of this work is given in the subsections below. For reasons of objectivity, the critique of the AEC's own program, given in the next five subsections, consists of verbatim statements by the Review Panel, which are then followed by additional brief comments by the Commission.

\section{1) General Comments on the AEC's Program}

The Review Panel makes the following statements:

"Under this program a broad attack is being waged, aimed at determining, under laboratory controlled conditions, the feasibility of power generation by fusion reactions. This requires as a first step the production and containment of dense hot plasmas. The attainment of this step is the immediate goal of the program. 
"Because of its complex behavior, plasma must be studied under a wide variety of conditions. Many of its salient properties emerge only when it is contained ir large volumes offered by large machines. Other important experiments are conveniently conducted in smaller size devices. The present structure of CTR research in this country is well proportioned. Considering the complexity of plasma as a fluid and the variety of techniques used in the studies, there is little duplication in the programs of the U. S. laboratories or in those of other countries, although there is enough desirable overlap to permit profitable comparison between results obtained by different groups.

"A wide variety of promising experiments are being pursued at the four major AEC-supported laboratories. Some of these are of quite recent origin while others have evolved naturally from lines of attack adopted early in the history of the program. Certain approaches have made the full round and have been discontinued. For example, the linear pinches, the toroidal Z-pinches, and their daughters, the stabilized pinches and hard-core pinches, have all served their purpose well and retired from the field. It is most gratifying to find that great strides have been made over the past four years in reconciling the theoretical understanding of plasma with its actual behavior in the laboratory in a number of experiments."

The AEC takes pride in the above comments by the Review Panel. It is clear, however, that the task of maintaining a wellproportioned program, with limited duplication of effort, will become increasingly difficult as the experimental devices grow in size. For small inexpensive experiments, appreciable duplication is sometimes appropriate, particularly for training purposes. For large and expensive experiments, however, the degree of justifiable overlap becomes much smaller. This problem will clearly require special consideration in future CTR activities.

$$
\mathrm{V}-4
$$




\section{Critique of the Program at PPPL}

The Review Panel made the following statements:

"The effort at the Princeton Plasma Physics Laboratory is devoted primarily to the production and confinement of hot plasma in toroidal (i.e., closed) systems of the stellarator type. Measurements of basic plasma properties in such devices have been extremely thorough and extensive. Diagnostic instruments and techniques of great value, such as the ion cyclotron reasonance heating method and the divertor, have been developed. Theoretical understanding of plasma physics has been advanced to a high level. Ingenious experiments in basic plasma physics have been performed in the $Q=$ machines.

"The Model $\mathrm{C}$ stellarator still represents the most valuable test bed in the world today for studying toroidal confinement. It will soon have serious competition, however, since the interest around the world in toroidal machines is increasing rapidly. The loss of plasma from this machine remains anomalously high and the underlying causes are not yet understood. Nevertheless, the series of studies just completed on how various heating methods affect the plasma loss rate have been invaluable in advancing our knowledge regarding the difficulties of toroidal confinement. The Model $\mathrm{C}$ stellarator is well engineered and efficiently utilized but somewhat inflexible."

During recent months it has become increasingly clear that plasma temperatures achievable in the $\mathrm{C}$ stellarator are being limited by the anomalously-high rate of particle loss. While not yet completely conclusive, the cause of this loss is widely believed to be due to one of several important classes of instabilities, or possibly to a mixture of them. Both in order to study these instabilities more effectively and to learn how to suppress them, it appears necessary to shift emphasis at this time to new or modified toroidal configurations 
incorporating high shear and/or minimum-B fields. In order to focus efforts on this problem of plasma confinement and stability, it may be desirable to halt work on new methods of plasma heating (such as magnetic pumping), at least temporarily .

(The action planned at PPPL, based on the policy of Section VI.A., is outlined in Section VI.B.2.)

3. Critique of the Program at ORNL

The Review Panel makes the following statements:

"The effort at the Oak Ridge National Laboratory is devoted to a variety of aspects of plasma research. In experiments directed at buildup of hot plasma via energetic charge particle injection into mirror machines, ORNL has been the leader. However, the complexity which arises from the large particle orbits in the $\mathrm{DCX}-2$ machine makes interpretation difficult, although considerable success has been achieved in understanding the plasma in the less ambitious DCX-1 machine. The beam-plasma interaction studies and hot electron plasma production via electron cyclotron heating have pointed the way in this type of experiment and have yielded results of considerable value. The measurement of basic cross sections has been invaluable.

"In the past, ORNL appears to have stressed the empirical approach to experimental plasma research. This method of attack may soon become unprofitable."

Experiments at Oak Ridge can and have yielded unforseen and unexpected results in plasma physics, providing a challenge for subsequent theoretical interpretation and thereby a growth of the science. Nevertheless, in a directed program such as CTR, it will be increasingly important to pursue those areas of work which permit a detailed comparison between theory and experiment. (For action planned at ORNL, see section VI.B.3.)

$$
\mathrm{V}-6
$$




\section{Critique of the Program at IASI}

The Review Panel makes the following statements:

"At the Ios Alamos Scientific Laboratory there is an unique involvement with pulsed dense high-beta* plasma experiments. The early work on straight pinches has been discontinued and the series of experiments on open-ended fast theta pinches has culminated in the highly impressive Scylla-IV. A number of ingenious diagnostic instruments have been incorporated with this experiment and many interesting results have been recorded. Valuable plasma gun developments have been made. The plasma focus device which produces intense thermonuclear reactions in a small volume is spectacular in its performance. Although many of the large-scale experiments we viewed were well engineered, the impression remained that the engineering support was inadequate for the type of program advocated. There appeared to be a considerable imbalance between theory and experiment. More intense theoretical effort is needed in the difficult regime of dense high beta plasmas."

Experiments with the Scylla-IV at Los Alamos show no evidence of any instabilities: plasma confinement, while admittedly brief, appears to be limited primarily by end losses. In order to avoid these losses, attention is now turning to the feasibility of constructing a toroidal theta pinch (Scyllac). If studies show that adequate equilibrium and stability can be expected for a high-beta plasma in a toroidal configuration, such a device would appear to be a tool of major importance in the study of plasma behavior.

*Beta is defined as the ratio of plasma pressure to magnetic field pressure. In low beta plasma the magnetic field which permeates the plasma is nearly the same as that in the absence of the plasma. In a high beta plasma the magnetic field inside the plasma is nearly zero. 
Certain other experiments at Ios Alamos - notably those involving cross-field injection and injection into cusps appear to be drifting somewhat. It would seem desirable to review these efforts, together with those directed toward producing miniature explosive fusion reactions, in the light of their relative contribution to the program as a whole.

(For action planned at IASL, see Section VI.B.4.)

5. Critique of the Program at IRL

The Review Panel makes the following statements:

"At the Lawrence Radiation Laboratory three major lines of attack are making rapid progress and providing encouraging results. These are the minimum-B geometry mirror machine, Alice, employing neutral injection; the E-layer experiment, Astron; and the toroidal experiment, the Levitron. The work on Alice, along with supporting experiments in more modest mirror machines, has played a leading role in reconciling stability theories with experiment and providing the scientific community with a larger measure of hope for the ultimate understanding and exploitation of plasmas.

"The performance of Astron has been impressive to date. It is an ambitious engineering undertaking and has demonstrated that its E-layer* is stable under conditions of modest field reversal. Astron has no counterpart; it is the only unique method being pursued by this country.

"The series of experiments recently completed in the Levitron have clarified a number of significant points on the behavior

*The term E-layer refers to very energetic (relativistic) electrons which form a cylindrical shell. The role of this shell is 1) to help produce a magnetic field which can confine the plasmas, and 2) to heat the plasma. 
of plasmas in toroidal configurations and pointed to several approaches which may ultimately circumvent the instabilities thwarting our efforts.

"The large mirror experiment called $2 X$ appears to be suffering from a succession of difficulties and its future prospects do not appear encouraging.

"The supporting studies in basic aspects of plasma physics and theory at both the Livermore and Berkeley sites are of high quality."

It is not yet clear to what extent end losses will limit the usefulness of open-ended devices as potential fusion reactors, even if all instabilities can be suppressed. It is clear, however, that open-ended systems can play a major role in the study of instabilities and of ways of suppressing them. Of particular value in this respect are steady-state systems such as Alice, in which the density is increased slowly by neutral injection until limited by an instability or some other loss mechanism which can then be analyzed in detail. Similar techniques may also become increasingly important in closed systems, such as the Levitron.

While results from pulsed systems such as $2 \mathrm{X}$ may be more difficult to interpret, they are expected to give valuable information concerning instabilities in the density range between that obtained to date by injection techniques and that of thermonuclear interest $\left(\sim 10^{14}\right.$ per cubic centimeter). 
With regard to the Astron, results to date have been encour-

aging. Although the weak E-layer thus far produced appears

to be completely stable, the question remains whether it will

continue to be stable as the electron density is increased

toward field reversal.

(For action planned at LRL, see Section VI.B.5.)

6. Critique of the Non-AEC Program

The Review Parel makes the following comments:

"The programs at the Naval Research Laboratory, at General Atomic, and at General Electric are all of high professional quality. The program at Aerojet-General Nucleonics is still in its infancy but appears to be well conceived and offers promise. The interaction between these programs and the more extensive AEC supported effort has been valuable and is to be commended. In particular, the theta pinch programs at NRL and GE have had significant influence on the Los Alamos effort. The development of novel neutral injection sources at AGN is being followed closely by workers at Livermore. The theoretical effort at GA has played an important role in CTR both nationally and internationally."

7. Critique of the AEC's University Program

The Review Panel makes the followirg comments:

"Work at the major AEC supported university plasma laboratories is yielding excellent retums in plasma physics and plasma diagnostic techniques for funds expended. The programs at Berkeley, at MIT, at Wisconsin and at New York University which were viewed by panel members appeared to be especially fruitful, with excellence in. output of research results and of gifted plasma scientists and engineers. Work at Stanford and at the Stevens Institute of Technology is contributing to our understanding of plasma physics."

Research carried out at university plasma laboratories constitutes a very important part of the AEC's program. Among 
other things, universities clearly play a critical role in the training of scientists and engineers needed to invigorate the CTR effort. While an expanded university program is an integral part of an intensified CTR effort, special attention must be given to maintaining high quality of the research work, with emphasis on theoretical studies of plasma behavior.

C. COMPARISON OF THE U.S. AND THE WORID EFFORT

At the time of the Geneva Conference of 1958, significant work in the field of controlled fusion was being carried out by only three countries in the world: The U.S.A., the U.K. and the U.S.S.R. Of these three efforts, that of the United States was the largest and most advanced, both in theory and experiment. Since that date, many other countries have entered the field*. Initially their research efforts invariably followed the lines of attack previously charted by the three leading countries. Their progress was rapid, however, due to their ability to draw heavily on existing knowledge. Nevertheless, as little as four years ago the United States was maintaining a position of real leadership in the field. As pointed out by the Review Panel, at that time the U. S. participation in CTR research constituted nearly one-half of the total effort in terms of weighted

* See Section III for a discussion of the countries involved in CTR work and of the types of experiments being pursued.

$$
\mathrm{V}-11
$$


expenditures and personnel and its effective contribution to the progress of the program amounted to well over one-half of the total.

In the intervening period, however, the relative role of the United States has decreased sharply. Specifically, as of now, the U.S. participation in the field is estimated to have dropped to one-fifth of the total world effort (see attached figure); furthermore, the effective contribution to progress is now estimated to be only about one-third of the total. This circumstance is in sharp contrast with the situation in many other major fields of physics research.

While the development of strong competition in controlled thermonuclear research is both healthy and desirable, the trend indicated by the above figures is alarming. If this decline in stature (relative to the rest of the world) is allowed to continue, it is obvious that the CTR program in this nation will soon deteriorate to a secondary role.

D. EXISTING BUDGETARY CONS IDERATIONS AND THEIR CONSEQUENCES

Impact on Manpower and Programs.

A key factor in the above development is the fact that during this four year period the budget for CTR research has been

$$
\mathrm{V}-12
$$




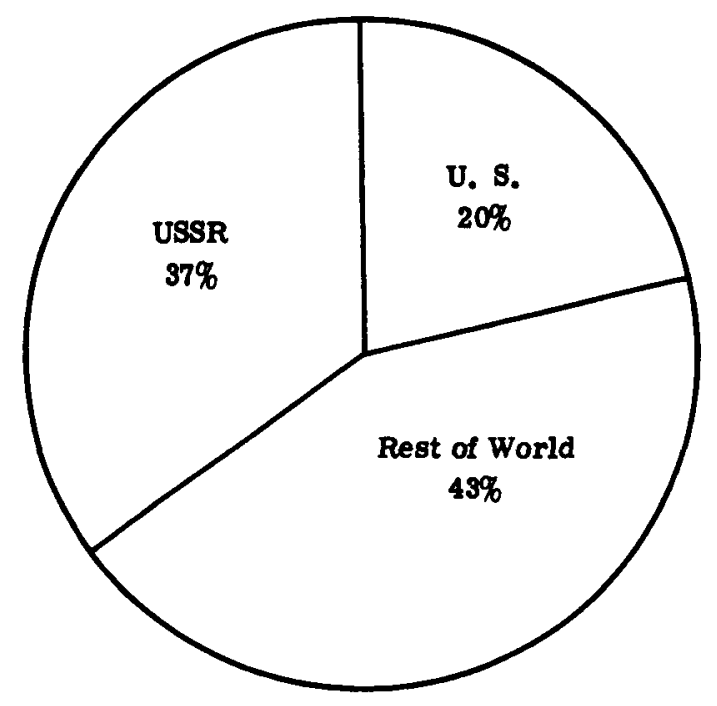

OPERATING COST HISTORY OF THE US AEC CONTROLLED

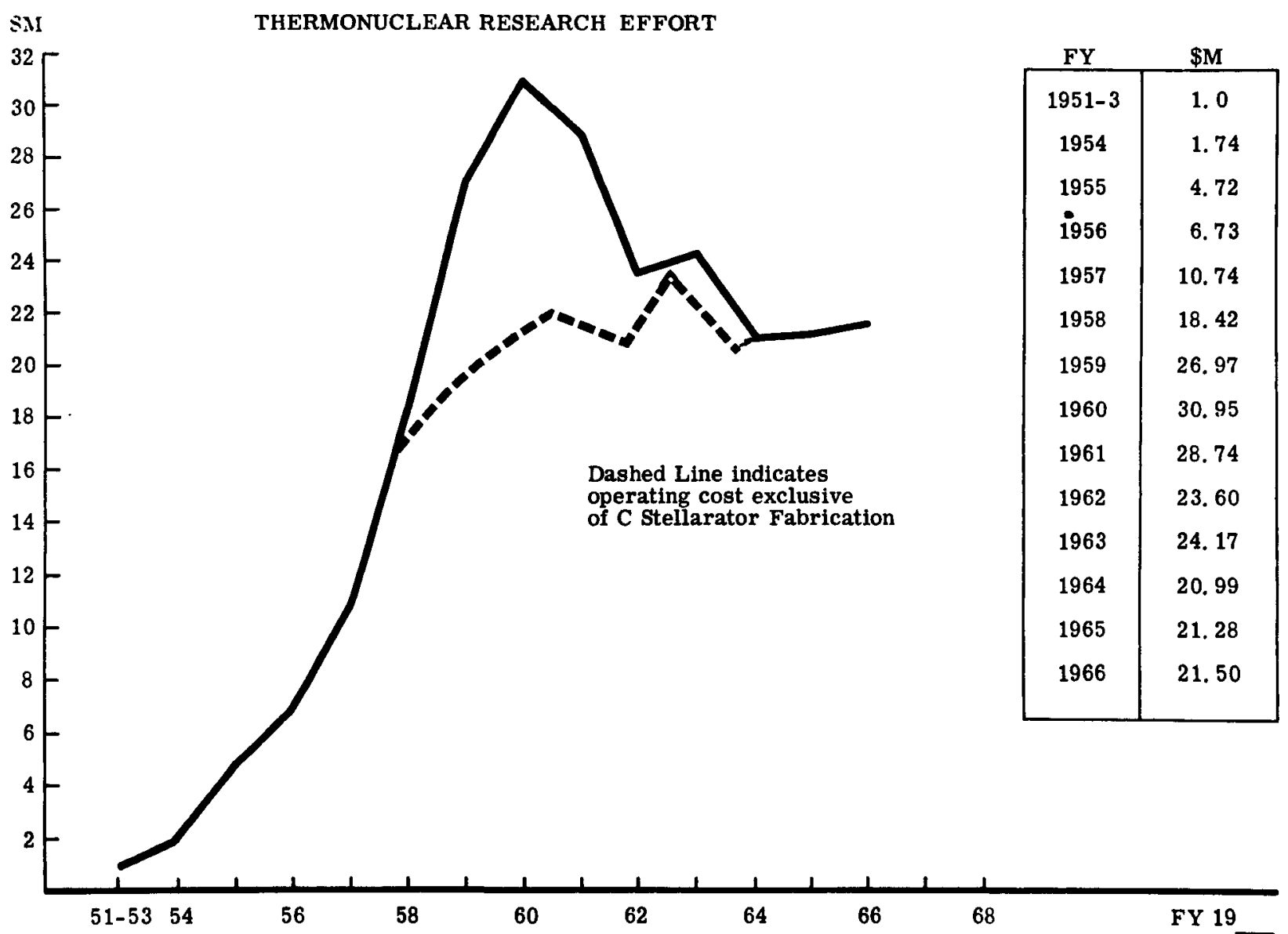


essentially static and largely inflexible. As a result, there has been a severe curtailment of the normal influx of new people with fresh ideas. Another result of the recent fiscal policies has been the lack in both speed and flexibility of adapting or acquiring equipment to test new ideas (see below) which have originated here and abroad. There has necessarily been a tendency to continue studies with equipment that is becoming out-dated, in an attempt to obtain maximum return from prior investment.

By contrast, the efforts in Western Germany, France, the U.K. and U.S.S.R. have expanded rapidly during this intervening period with new devices, new facilities, and vigorous youthful staff. During the same period, intensive programs were created in Japan, Italy, and a number of other countries.

\section{New and Neglected Areas}

Fusion research has a number of new areas that need to be investigated, and of interesting new ways of attacking existing problems. Enough is now known about plasma physics to weed out the unpromising or the obviously impractical. Even after the weeding-out process, however, the list of new or neglected areas that should be pursued has been growing. 
Iisted below are some of the topics that are either a) now

absent in the U.S. fusion program, or b) have been allowed to

lie fallow for lack of budgetary support, or c) are in the

category of new ideas. It should be noted that some of these

are already under intensive study in foreign programs.

1. Dynamic Stabilization

The whole area of dynamic (non-passive) means of plasma stabilization, including the effect of high frequency fields on plasma stability and confinement is virtually absent from the U.S. fusion program.

2. Shock and Turbulent Heating; Instability Heating

One of the most active fields of research today (in foreign programs) concerns the question of heating plasmas to thermonuclear temperatures by shocks, plasma turbulence or by controlled plasma instabilities. Such studies not only could have great significance to fusion, but also bear on aspects of upper atmospheric and related effects, that are of substantial scientiric and practical value. There is little work in progress in this field in the U.S. fusion program.

3. "Average Minimum-B" as applied to open-ended systems

The success of the minimum-B principle in open systems suggests the value of also testing the "average minimum-B" idea in such systems. In addition to practical advantages, such tests might provide early, inexpensively obtained, answers to some of the questions concerning the use of "average minimum-B" in toroidal systems. Essentially no work of this kind is underway in the U.S. program.

4. Special Technological Developments

There are severalareas of technological developments that are not being actively pushed in the U.S. program, for lack of adequate budgetary support. The end result of the inadequacy of support has been that either (a) it has been necessary to "make do" with older technology, 
or (b) the problem has been put off until it becomes intolerably acute, and it has not yet reached that stage. Examples are, respectively: (a) the development of new types of high intensity ion and neutral beam sources; (b) many questions having to do with the environment of a plasma, particularly the walls and their role.

\section{E. CONS IDERATION OF A NATIONAL CENTER}

As a result of the above-mentioned concern over the decreasing stature of the U.S. effort (particularly in a program which is gathering momentum throughout the world), extensive consideration has been given to methods of reversing the present trend. In addition to the obvious implications on budgetary requirements for this program, it seemed desirable to find other forceful methor.s of invigorating the U.S. effort.

One of the strongest recommendations made by the Review Panel

was that the Commission should take immediate steps toward establishing a National Center for Plasma Studies and Controlled

Fusion. As envisaged, the function of such a center would be:

1. To extend and stimulate the controlled thermonuclear program in this country. In this respect, it would supplement (rather than supersede) the existing national laboratories engaged in this work.

2. To permit the development of a wide variety of different experiments at the same site. Such a development would result not only in important cross-fertilization of concepts and techniques, but also in providing the capability for rapid construction of new experiments, drawing on the broad competence available there. A capability of this nature would be particularly important when and as really large-scale devices are deemed justifiable. 
3. To serve as a major center for national and international exchange in the effort leading to controlled fusion. In this way, it would automatically result in a continuing flow of fresh new blood into the program, both from within this country and abroad. This influx of new and young people would correspondingly bring the flow of fresh ideas needed to invigorate the U.S. effort. It is recognized that to be effective in this goal, such a National Center would necessarily have to be free of all security restrictions.

4. To promote close contact between universities and the controlled fusion program and to implement the training of scientists and engineers in this field of research. This end would be accomplished through the institution of cooperative graduate and postgraduate programs, and through visiting and summer appointments for university scientists and engineers.

Clearly there are many questions and problems which arise in connection with the development of such a center - problems which will require careful study and long-range planning. These problems are discussed in section VI.B.8.

\section{F. OVERALL CONCLUS IONS}

Scientific and Technical

Research in controlled fusion and the physics of high temperature plasmas has now become an established scientific discipline. World progress toward a detailed understanding of the behavior of high temperature plasma is rapid and accelerating, so that fusion research, initially a largely-empirical effort, is fast becoming a quantitative science. Both in scope of effort and in level of sophistication of theory and experiment, there has been a major change in the last four years. 
Technical problems that have in the past represented serious impediments to fusion research are being solved, and new technological developments are having a major and favorable effect on the research. As an example, the problem of plasma impurities has been brought under effective control in many cases, through the use of newly-developed vacuum techniques, plasma divertors, and other means. The creation of plasma at thermonuclear temperatures, formerly a goal in itself, is now routine in many experiments. Superconducting magnet coils have been developed and are beginning to be tested in fusion experiments. The effect of such coils in future experimentation is expected to be very important. Plasma diagnostic methods are now extensive and permit measurement of all of the important properties of a plasma.

The world experimental and theoretical effort in fusion research is now being focused almost exclusively on the key scientific issue - an adequate understanding of the stability properties of magnetically confined plasmas, on which all future practical applications of such plasmas hinge. Major steps have been taken, both toward understanding and toward control of plasma instabilities. Experimentally, developments include: (a) The conclusive documentation of the effectiveness of "minimum-B" magnetic wells in stabilizing 
an important class of plasma instabilities, (b) The confirmation in specific important cases of the validity of the basic equations now used to predict plasma instablilties. Marked progress has been made in developing theory which better represents real situations, and in predicting the actual effects of plasma instability and turbulence. Guidelines, based on sound thermodynamic arguments, have been developed for predicting the "most-likely-to-be-stable" plasma confinement conditions.

While no definite time-table can be established for determining the scientific feasibility of controlled fusion power, the rate of approach toward this goal is clearly accelerating.

\section{Adm1nistrative}

In view of the complexities of the problems and the scarcity of funds, it is essential that effective coordination and cooperation exist among scientists in this field. This statement applies not only to activities which are AEC sponsored, but also to interactions with foreign programs. The entire U.S. effort has unquestionably been hampered by a lack of effective coordination and cooperation among the four AEC laboratories; to a lesser extent, it has also suffered from restrictions which have been placed on foreign scientist participation at three of the four laboratories.

$$
\mathrm{V}-18
$$


Implications Concerning the U.S. Program

The possibility of fusion power captures the imagination, and its eventual economic impact could be of major significance. From the scope and nature of fusion programs in other major nations, it is evident that this field of research provides a focus for the whole new scientific field of high temperature plasma physics. The United States was one of the founders of fusion research, and made the bulk of the original contributions to this field. Though the fusion problem is more complex than originally thought, years have not diminished the importance of finding out if it can be solved, and if so, what its impact would be.

During recent years, however, the United States has been losing its momentum in fusion research, vis-a-vis other nations, due largely to the effect of a static budget and the many consequences thereof. Definitive steps must be taken to reverse the present trend. 
Drawing upon the findings summarlzed in the above section, the Commission presents below the action which, in its view, is required in order to ensure a timely and responsible evolution of the controlled thermonuclear program. This action consists of two parts: A) the establishment of a set of broad policy considerations designed to guide further developments in the program, and B) specific steps toward implementation of this policy.

\section{A. Policy Statements}

1. The United States program in controlled thermonuclear research is clearly of major scientific and technological importance to the nation as a whole, and should be supported at a level which will ensure that the nation maintains high competence in the field.

2. The AEC program will continue to be motivated by interest in eventually achieving controlled thermonuclear power. It is recognized, however, that there are many other benefits which will accrue from an investigation of this vast and largely-unexplored field. As a result, the effort will emphasize not only a detailed understanding of the physics of high temperature plasmas and the means for confining and heating them, but also studies of a basic nature in the broader aspects of the science and technology of plasmas.

3. The overall responsibility for the development of controlled thermonuclear power in the United States clearly falls under the jurisdiction of the Atomic Energy Commission. Since, however, other government agencies have interests in specific aspects of the field, their collaboration in the support of the program is both appropriate and welcomed.

\section{B. Specific Steps to be Taken}

1. Concerning the AEC's Program as a Whole.

The determination to maintain a high degree of competence in the controlled thermonuclear program will require a significant strengthening of the AEC's overall effort in this field. As now envisaged, the major requirements are the following:

$$
\mathrm{VI}-1
$$


a) Scientific-technical: An intensification of the experimental and theoretical effort, both at the major CTR laboratories and at universities. A number of large new experimental devices are now urgently needed within the program in order to test recent concepts for improved plasma confinement. The plans for this work, as well as for other supporting research on the behavior of plasmas, are outlined in some detail in the sections below. These developments will require a concomitant growth in the scientific and technical manpower engaged in the effort.

b) Administrative: Greatly increased coordination and cooperation within the CTR program as a whole. The cost of carrying out all of the plans indicated as desirable elements of the program would far exceed the funds reasonably expected to be available. 1 / Careful consideration must be given to the choice of new projects to be supported, and to the continued effectiveness of those in existence. Both to obtain guidance on these and other matters and to ensure a close cooperative effort within the overall program, the Division of Research is establishing a CTR Standing Committee (and supporting ad hoc panels) composed of members from each of the major laboratories and from the scientific community as a whole. The structure and responsibility of these groups are outlined in Appendix 7.

1/ As of April 1966, estimates of additional operating funds needed by the CTR laboratories solely for fabrication of major new experiments during FY 1968, FY 1969, and FY 1970 total almost $\$ 6$ million per year. Examples of such devices which are in an advanced state of planning are: Scyllac (LASL), Alice II (LRL), improved Astron In jector (LRL), Toroidal Multipole (Princeton), and Superconducting Levitron (LRL). 
c) Financial: The operating funds required for this program consist of two parts:

(i) Funds neeajed for the fabrication of large new experimental devices designed to test the latest theories of plasma confinement. Such devices are essential to further progress in controlled fusion research. When and as required, each will be justified on a case-by-case basis. The total cost of fabrication is expected to be 3 to 4 million dollars annual1y. (ii) Funds needed for normal operation of the CTR effort. In addition to financing the on-going research program, these funds must be sufficient to permit (1) an intensive experimental effort using the above new devices, (2) the influx of badly-needed new scientists into the program, both at CTR laboratories and particularly at universities, and (3) the financing of small-scale experiments associated with this personnel increase. The types of experiments envisioned in (1) and (3) above are summarized on pages 5 through 8 of this section. In partial compensation for the cost of an intensified effort with large new devices, activities with some of the earlier experimental equipment will correspondingly decline in $v$ alue and can eventually be cut back or eliminated. The CTR Standing Committee will work with the Division of Research in helping to identify such activities. 
The accomplishment of the above-mentioned goals will

require a net increase of about $15 \%$ per year in norma1 operating funds over the next five years. This is in addition to the cost for fabricating major new devices discussed in (a) above.

$* * * * * * * * * * * * * * * * * *$

In connection with paragraph (a) above, a major intensification

of effort is clearly indicated in each of the following broad areas of research:

a) The development of experiments which are specifically designed to isolate and study a single type of instability of importance to the CTR program.

b) Investigations of the confinement of plasmas in absolute minimum-B systems.

c) A study of the effect of controlling particle energy distributions on the development of plasma instabilities.

d) The study of toroidal systems possessing average minimum-B configurations.

e) A study of the equilibrium and stability of high beta plasmas in toroidal devices.

f) The application of digital and/or analogue computers to the prediction of plasma behavior, under conditions as realistic as possible, either through simulation or through solution of the plasma equations.

g) The study of non-1inaar theory. 
In addition, programs involving participation by graduate students and visiting scientists will be expanded at the AEC sites, as appropriate.

The specific plans for programs at each of the CTR laboratories and off-site projects are outlined in sections B2 through B8 below. The manner and priority in which these plans will be carried out will, of course, be greatly influenced by the views of the CTR Standing Committee.

2. At the Princeton Plasma Physics Laboratory.

The action planned by the Princeton Plasma Physics Laboratory includes:

a) Concentration of the research effort toward understanding the reasons for anomalous diffusion from stellarators and other toroidal devices. In this connection, particular attention will be given to:

1) The problem of modifying the present configuration of the stellarator into one possessing an average minimum-B and/or a very high shear.

2) A comparison of the various proposed average minimum-B geometries on the basis of existing (though incomplete) theory, while at the same time working toward a more adequate theory.

3) The design and eventual construction of an average minimum-B device which appears best according to the above theoretical analyses.

4) Investigation of the possibilities of establishing the minimum-B and shear stability requirements of a torus by performing suitably designed experiments in simpler linear geometry. 
b) Intensification of the effort on the development and/or improvement of methods for heating plasmas confined in closed systems. Such methods include not only ion cyclotron resonance heating and magnetic pumping, but also less conventional candidates (e.g., energetic plasma injection via neutral beams or plasma guns, hybrid schemes of turbulent heating).

c) Expansion of the already existing program of basic plasma studies.

3. At the Oak Ridge National Laboratory.

The action planned by the Oak Ridge National Laboratory

Includes :

a) Intensification of the experiments toward the accumulation of hot, dense plasmas by high-energy injection, with emphasis on the steady state. This is expected to involve stability studies related to theory in appropriate ways, the development of intense particle beams, the development of suitable magnetic configurations and trapping methods, and pertinent studies of the basic atomic physics upon which the various trapping processes are founded.

b) An intensification of the theoretical effort, with emphasis on relation to experiments.

c) The further development of a strong program in basic plasma physics.

d) A continuation of the studies of turbulent heating and of the properties of the electron-cyclotron plasmas.

e) A continuation of the development of technical and engineering programs related to controlled fusion, including magnetic field engineering, vacuum technology, and the evaluation of problems arising from the interaction of hot plasmas with their material environment.

f) The continuing development of the Instruments of plasme physics, directed to the ready and definite diagnosis of the complexities of plasma behavior. 
g) The continued search for novel ways of heating and confining dense plasmas.

4. At the Los Alamos Scientific Laboratory.

The action planned by the Los Alamos Scientific Laboratory

includes:

a) Intensification of work on high beta plasma. In this connection, particular attention will be given to the rapid strengthening of the theoretical and engineering support.

b) Continuation and completion of the feasibility study of a toroidal theta pinch (SCYLIAC). If the project is found feasible, appropriate steps will be taken toward the design and eventual construction of this facility.

c) Continuation of work on hydromagnetic guns, in anticipation of achieving about a factor of 1000 in intensity. Continuation of studies of injection into transverse and parallel magnetic fields.

d) Continuation of work on the dense plasma focus with the intent of finding the underlying principles and of maximizing the product nt.

e) The establishment and then rapid strengthening of an effort in basic plasma physics.

5. At the Lawrence Radiation Laboratory.

The action planned by the Iawrence Radiation Laboratory

includes :

a) Intensification of confinement and stability studies of plasmas produced by the injection into minimum-B systems of energetic neutral hydrogen atom beams, spread in energy ،

b) Emphasis on research aimed at establishing correlations between theory and experiment in toroidal systems with particular emphasis upon the effectiveness of field configurations combining minimum-average- $B$ with shear in avoiding plasma losses due to instabilities. 
c) Continuation at a vigorous pace of the experimental and theoretical investigation of the Astron concept. Emphasis will be placed on studying the stability characteristics of the E-layer. Early attention will be given to the construction of the proposed new Astron accelerator which is designed to give a significantly higher electron output than is now available.

d) Extension of the theory of wave-particle instabilities as applied to open-ended and closed confinement systems.

e) Expansion of studies in basic plasma physics and atomic processes basic to the CTR effort.

6. Regarding Work at Non-AEC Sites.

The action planned by the AEC includes:

a) Continuation of friendly and active contact between its program and allied efforts outside its immediate cognizance.

b) Steps to ensure minimum duplication of effort.

c) Steps to encourage exchange of information between AEC and non-AEC scientists.

7. Regarding AEC-Supported Work at Universities.

The action planned by the AEC includes:

a) A significant expansion of the AEC-supported effort at universities. Specifically, it is planned to expand the support of these activities at a more rapid rate than that of the national laboratories. The university support will extend to basic plasma studies, recognizing that a thorough understanding of the field will be required for achievement of the ultimate objective.

8. Regarding the National Center.

As pointed out in Section V-E, the Review Panel strongly recommended the establishment of a National Center for Plasma Studies and Controlled Fusion Research. Among the more important benefits which could be expected to accrue from the 
existence of such a center are: a) that important crossfertilization of ideas and techniques would result from having a sizeable number of experiments located at the same site, b) that the possibility of broad and unrestricted exchange of scientific personnel on a national and international scale would serve as a major stimulus to the program as a whole and would help greatly to maintain a position of leadership in the field, and c) that when really large-scale containment devices are deemed justifiable, the center would be in a good position to move ahead rapidly toward their construction, drawing on the broad technical competence it would have developed through smaller experiments.

While these are all valid reasons for establishing a National Center, it is clear that many of the above benefits can be achieved more simply — and far less expensively - by a strong cooperative effort within the existing program itself. As pointed out in Section VI.B.1., steps are now being taken to establish a closely integrated program through the medium of a CTR Standing Committee. Among other things, this Committee will actively encourage both a cross-fertilization of ideas and a broad exchange of personnel among the present sites. (Efforts will also be made to free all CTR sites from security 
restrictions, so that access to these areas is readily avallable to scientists both from this country and abroad.)

The development of a strong coordinated program will thus remove much of the urgency for establishing a National Center in the CTR field. It is well recognized, however, that such a center could well serve a useful purpose at some future date, when and as really large-scale containment devices are deemed justifiable. With this in mind, the Standing Committee will eventually be expected to consider such questions as: 1) the way in which a National Center could best be established with the maximum possible benefit to the program as a whole, 2) the relative advantages and disadvantages of establishing it at one of the existing CTR sites (versus a wholly new location), 3) the sort of time scale which would seem most reasonable for its development, and 4 ) the impact of such a center (once established) on the existing CTR laboratories. 


\section{Appendix 1}

\section{The 1962 Report of the General Advisory Committee}

\section{Conclusions and Recommendations}

In the Sherwood program there remain many unresolved technical questions that can be decisive to the feasibility of controlled fusion as a source of economic power. These relate particularly to the problems of stable confinement of hot plasmas in configurations suitable for power, to methods of initially heating the plasmas, to problems of purity, etc. However, it appears that, if these feasibility problems can be overcome, controlled fusion power could be economically competitive at least with breeder reactors. For this reason we believe that the AEC should continue a vigorous program in controlled fusion research and in basic research in hot plasmas. However, this recommendation should be reviewed periodically since its continuing validity is dependent upon future technical developments.

The crucial problem of the Sherwood Project is the creation of a stable confined plasma, at thermonuclear densities and temperatures. To date, it is not certain that the stability question is a soluble one although there are some reasons for optimism. Although a vast morphology of instabilities has been uncovered, exhaustive theoretical work has not turned up any universal instability which would make

$$
\text { A- I- } 1
$$


such confinement unattainable. However, the complexity of the problem is such that this argument is not conclusive. It should be borne in mind that many heating schemes, such as onmic heating, have been shown to be unstable both theoretically and experimentally.

To date, there has been at least one Sherwood experiment - Table Top - which has produced plasmas almost at thermonuclear conditions, which has been confined for periods which are at least very long when compared to the fast-growing instabilities which have bedeviled other experiments.

A prime objective of the Sherwood program must be further study of stable plasmas with a view to understanding and extending the regimes of stability. It seems likely that the ultimate fusion reactor will be a closed system such as the Stellarator; thus, some work on closed systems must be continued. However, the vastly greater ease of injecting into, heating and diagnosing the simple open-ended systems, mirror and cusp, make these indispensable tools for plasma study at this time. It is also important to keep in mind that many of the confinement schemes now used for stability studies would not be adaptable to use in a full-scale fusion power system, even if capable of providing stable confinement. It should be emphasized that characteristic times for instability are as much as a factor $10^{8}$ shorter than thermonuclear times, so that, a "leaky" confinement which is not quite adequate for fusion economics may still be very suitable for stability studies. Only after the 
fundamental existence theorem for hot stable plasmas has been proven should primary emphasis be placed on evaluating confinement schemes relative to their potential as ultimate reactors.

In FY 1961, the Commission spent $93 \%$ of its controlled fusion research money in but four laboratories, while on $1 y$ $7 \%$ was spent in all other research institutions. We believe that the need for new ideas and additional knowledge is such that increased numbers of creative individuals should be involved in the AEC-supported programs. Both the California and Princeton groups emphasize they have recently found that graduate students can make contributions of particular value to Sherwood research programs. We fully support this conclusion, but we note that there are many other institutions with well-qualified graduate students who are not reached by the AEC programs in its four principal Sherwood laboratories. It appears to us that as much as $25 \%$ of the AEC Sherwood research funds could be used to support research of well-qualified groups of scientists outside the four main Sherwood laboratories.

The entire Sherwood project suffers from insufficient cooperation among the major sherwood laboratories, both in the planning of research and specific technical cooperation. It is important that cooperation be increased. The achievement of such cooperation may require an increased effectiveness in the AEC management, it should be attainable - and very worthwhile. For example, at the present time A - I - 3 
the Oak Ridge laboratory could probably provide valuable help to Princeton in the engineering of magnetic pumping, and Los Alamos might well provide plasma guns for the Stellarator, or at least scientists to develop such guns. Likewise, the laboratories could provide effective and constructive criticism of all proposals for major new devices.

The AEC-supported Project Sherwood program is producing important and useful results at a higher rate than before and we expect that valuable results will continue to flow from this effort. Furthermore, recent engineering studies of possible thermonuclear reactor configurations have reinforced the opinion that, if it is possible to obtain a hot confined plasma, it may be possible to produce electricity at costs of the order of those which will pievail in thermal or nuclear plants in the same time period.

\section{A Summary of Principal Recommendations}

We recommend that the AEC -

1. Continue to support Project Sherwood vigorously.

2. Require that the Princeton Stellarator Project produce a plasma in the kev temperature range with a ratio of material pressure to magnetic pressure, $\boldsymbol{\beta}$, of at least $1 \%$ within three years, or, if unable to do so, the Model C portion of the Princeton program should be closed out. Plasma confinement by the Stellarator principle, or a related closed geometry, appears to be necessary for a practical

$$
\text { A }-\mathrm{I}-4
$$


thermonuclear power system. It is therefore, unfortunate that the Princeton organization has not been adequately effective in dealing with the major engineering and construction problems of the stellarator project. Men experienced in constructing major hardware (examples are Livingston, Jacobus or Green) should be brought into the Princeton project and given a reasonable time either to get the Model C Stellarator into satisfactory operation or demonstrate that it cannot be done.

3. Redistribute the effort at Oak Ridge by (1) shutting down the DCX-1 facility as soon as feasible (2) emphasizing the DCX-2 and increasing the strength of experimental and theoretical physics effort on the sherwood program.

4. Determine that Astron is not part of the AEC Sherwood program. Transfer responsibility for the Astron accelerator to ARPA for DOD-supported research programs.

5. Discontinue construction of the Toy Top $2 \mathrm{X}$ experiment until such time as there is clear-cut justification from Toy Top and Table Top experiments currently planned (especially in regard to stability) for going to such a large scale in the magnetic compression and transfer work.

6. Increase moderately the size of the LASL program provided this can be done without increasing the size of the Laboratory or decreasing the weapons effort. 
7. Expand support of high-quality plasma research at universities. This could desirably constitute as much as $25 \%$ of the total Sherwood budget.

8. Support high-quality investigations of the systems engineering, chemical and other non-plasma development problems of full-scale fusion power plants. Systematic investigation of these non-plasma problems is an essential element of a complete fusion power development program. This effort could desirably constitute as much as $5 \%$ of the total sherwood budget.

9. Explore means for making the AEC management of the Sherwood program more effective, in view of the changed character and emphasis of the program. 


\author{
Appendix 2 \\ REPORT OF REVIEW PANEL ON
}

CONTROLLED THERMONUCLEAR RESEARCH

December, 1965

The Controlled Thermonuclear Research Review Panel acknowledges its deep indebtedness to its first Chairman, Dr. Samuel K. Allison. Under his guidance, the panel made visits of two to three days duration to Princeton, Oak Ridge, Livermore and Berkeley, and the Los Alamos sites. At each laboratory, scientists made presentations to the panel. Before each visit, extensive background reports prepared by project scientists were studied by members of the panel.

Presentations were also heard frnm members of the thermonuclear research groups of the General Electric Company, General Atomics, the Naval Research Laboratory and the Aerojet-General Corporation. Subgroups of the panel visited the laboratories at MIT, at the Stevens Institute of Technology, and at the University of Wisconsin. Presentations were heard from the New York University theoretical group, from the Stanford University research group, and from several scientists not now formally associated with CTR. Three members of the panel, Drs. Samuel K. Allison, S. J. Buchsbaum, and T. H. Johnson attended the Second International Conference on Plasma Physics and Controlled Thermonuclear Research at Culham, England, September 6 to 10, 1965.

As a result of this study, the panel has arrived unanimously at a number of findings and recommendations which follow. 


\section{PROGRAM JUSTIFICATION}

(1a) We find that:

The portion of the 1954 directive (AEC 532. 15, January 15, 1954) stating, "Controlled thermonuclear reactions research will be intensified and directed toward the development of a controlled thermonuclear reactor which will provide a source of useful energy for power..." has served and can continue to serve for justification of the AEC thermonuclear program and as a useful guide for program planning. The portion of this directive which continues, "... as well as a source of neutrons potentially competitive with fission reactors in producing fissionable materials." has become partially outmoded at this time.

The nation must know what possibilities lie in the thermonuclear domain. Future generations will obtain their power from uranium and thorium mined from rock or from heavy hydrogen extracted from the sea. At this stage both routes must be followed. Compared with the more advanced fission reactors, fusion reactors have potential advantages: their waste is not radioactive, their fuel is more readily obtained, and their product is less easily diverted to weapons.

The vision of limitless power for generations to come has stirred the imagination of people everywhere. This vision is a spur to scientists in this program throughout the world. All major industrial nations are exploring CTR with vigorous programs. We must participate actively in this effort in order that we may develop the technical competence required to expivit forthcoming results. 
Plasma may be viewed as a fourth state of matter. The sun is composed of it, the earth and the other planets of the solar system are bathed in it; plasma fills the vast regions of interstellar space. Its properties have received little attention until now. Its bearing on other fields of knowledge and the future activities of mankind may be profound. We must not remain ignorant of its properties.

(1b) We recommend that:

The Atomic Energy Commission continue its policy of support for Controlled Thermonuclear Research on a scale that will assure continued U.S. leadership and that it direct its efforts in this fleld broadly towards a thorough understanding of high temperature, high density plasma, the means for confining and heating, it and towards the eventual demonstration of the feasibility of controlled thermonuclear fusion reactions for the generation of electric power.

(2a) We find that:

AEC fiscal documents in which the CTR program is presented as five approaches to the generation of thermonuclear power do not properly represent the present course of the program. Under this program a broad attack is being waged, aimed at determining under laboratory controlled conditions the feasibility of power generation by fusion reactions. This requires as a first step the production and containment of dense hot plasmas. The attainment of this first step is the immediate goal of the program. 
Because of its complex behavior, plasma must be studied under a wide variety of conditions. Many of its salient properties emerge only when it is contained in large volumes offered by large machines. Other important experiments are conveniently conducted in smaller size devices. In the opinion of the panel the present structure of CTR research in this country is well proportioned. Considering the complexity of plasma as a fluid and the variety of techniques used in the studies, there is little duplication in the programs of our own laboratories or in those of other countries, although there is enough desirable overlap to permit profitable comparison between results obtained by different groups.

(2b) We recommend that:

The AEC fiscal and program descriptive documents be revised to reflect the point of view that several diverse laboratory programs important to the science and technology of plasma are being pursued within its CTR effort. In order to distinguish the programs, it may choose to describe them as confinement schemes using either open-ended (i.e. mirror) or closed (i.e. toroidal) configurations, as long time (i.e. steady-state) or short time (i.e. pulsed) confinement, as tenuous (i.e. low beta) or dense (i.e. high beta) plasma, and as supporting studies involving a large host of topics such as injection with energetic neutral beams or plasma guns, turbulent interactions with beams, currents and collisionless shocks, instability studies, plasma diagnostics, and the physics of surfaces involved in plasma reflux, ... to mention but a few. 
(3a) We find that:

A wide variety of promising experiments are being pursued at the four major AEC supported laboratories. Some of these are of quite recent origin while others have evolved naturally from lines of attack adopted early in the history of the program. Certain approaches have made the full round and have been discontinued. For example, the linear pinches, the toroidal $Z$-pinches, and their daughters, the stabilized pinches and hard core pinches, have all served their purpose well and retired from the field. It was most gratifying to find that great strides have been made over the past four years in reconciling our theoretical understanding of plasma with its actual behavior in the laboratory in a number of experiments. We find that CTR is rapidly moving from an empirical art into a quantitative science.

(3b) We recommend that:

The AEC continue to rely for the immediate future upon the four major laboratories for the bulk of CTR effort, that it support energetically a number of current experiments and novel excursions which we set forth, that it be prepared to augment this effort in ways we describe later, and that it exercise courageous management in terminating and redirecting approaches which reach the point of diminishing return. 
(4a) We find at the Princeton Plasma Physics Laboratory:

An effort devoted to the production and confinement of hot plasma in toroidal (i. e. closed) systems of the Stellarator type. Measurements of basic plasma properties in such devices have been extremely thorough and extensive. Diagnostic instruments and techniques of great value such as the ion cyclotron resonance heating method and the divertor have been developed. Theoretical understanding of plasma physics has been advanced to a high level. Ingenious experiments in basic plasma physics have been performed in the $Q$-machines.

The model C-Stellarator still represents the most valuable test bed in the world today for studying toroidal confinement. It will soon have serious competition, however, since the interest around the world in toroidal machines is increasing rapidly. The loss of plasma from this machine remains anomalously high and the underlying causes are not yet understood. Nevertheless, the series of studies just completed on how various heating methods affect the plasma loss rate have been invaluable in advancing our knowledge regarding the difficulties of toroidal confinement. We find the model C-Stellarator well engineered and efficiently utilized but somewhat inflexible. 
(ab) We recommend that.

The present series of experiments directed toward improved ion cyclotron heating in the model C-Stellarator be carried out rapidly and that the Model $C$ then be modified to provide the minimum average $B$ property.* This modification is in line with current theories regarding the beneficial effects on plasma stability of minimum average B. PPPL should continue the development of apparatus for magnetic pumping for its ultimate utilization on the modified C-Stellarator.

The recommended modification of the C-Stellarator should be regarded as only a first step toward a new generation of toroidal experiments which are presently evolving as a result of progress in our understanding of plasma stability in toroidal geometries. The AEC should not hesitate to support more than one major excursion in the study of toroidal confinement since the potential rewards are great indeed. Not only is it of interest to investigate a variety of postulated stable schemes, but it is important to have at hand more than one way of preparing the high temperature plasma which

* "Minimum B" refers to a configuration in which the absolute value of the magnetic field is nonzero and increases in all directions from some point. A plasma in such a magnetic well possesses exceptional hydromagnetic stability. In a closed, that is, toroidal system, a "minimum-B" well is topologically impossible in vacuum. The next best thing is to ensure that a particle experience on the average a "minirnum-B" configuration. 
is destined for 1 ong term confinement in the toroidal bottle. Such candidates as energetic plasma injection via neutral beams or plasma guns, and hybrid schemes of turbulent heating should receive careful consideration in addition to more conventional methods of in situ heating.

Support of research in basic plasma physics and theoretical studies should be continued at a generous level. The close contact which exists between PPPL and the teaching staff of one or two departments at the university is welcome but is too sparse. The interaction with the university should be enlarged to embrace a wider spectrum of the physical and engineering sciences.

Freedom from security restrictions at Princeton has been of great benefit not only to the Princeton program but to the overall CTR program. The ability to host U.S. and foreign scientists has filled a necessary requirement for the exchange of information with the rest of the world. More extensive exchange visits should be sponsored. 
We find at the Oak Ridge National Laboratory:

An effort devoted to a variety of aspects of plasma research.

In experiments directed at build up of hot plasma via energetic charge particle injection into mirror machines, ORNL has been the leader. However, the complexity which arises from the large particle orbits in the DCX-2 machine make interpretation difficult, although considerable success has been achieved in understanding the plasma in the less ambitious DCX-1 machine. The beamplasma interaction studies and hot electron plasma production via electron cyclotron heating have pointed the way in this type of experiment and have yielded results of considerable value. The measurement of basic cross sections has been invaluable.

In the past, ORNL, appears to have stressed the empirical approach to experimental plasma research. This method of attack may soon become unprofitable.

(5b) We recommend that:

ORNL emphasize research and development of intense ion sources and techniques for injection and energetic plasma production. The effort should stress neutral beam injection embodying advanced innovations leading to spreads in energy and momentum as dictated by current and improving theories.

Beam-plasma interactions leading to turbulent heating and burnout, and electron cyclotron heating of plasmas should be continued vigorously. Renewed effort toward increasing the theoretical staff and coupling it closely to the experimental program should be undertaken. 
(6a) We find at the Los Alamos Scientific Laboratory that:

There is an unique involvement with pulsed dense high beta* plasma experiments. The early work on straight pinches has been discontinued and the series of experiments on open-ended fast theta pinches has culminated in the highly impressive Scylla-IV. A number of ingenious diagnostic instruments have been incorporated with this experiment and many interesting results have been recorded. Valuable plasma gun developments have been made. The plasma focus device which produces intense thermonuclear reactions in a small volume is spectacular in its performance. Although many of the large scale experiments we viewed were well engineered, the impression remained that the engineering support was inadequate for the type of program advocated. There appeared to be a considerable imbalance between theory and experiment. More intense theoretical effort is needed in the difficult regime of dense high $\beta$ plasmas.

* Beta is defined as the ratio of plasma pressure to magnetic field pressure. In low beta plasma the magnetic field which permeates the plasma is nearly the same as that in the absence of the plasma. In a high $\beta$ plasma the magnetic field inside the plasma is nearly zero. 
(6b) We recommend that:

The effort on high beta plasmas be supported strongly and that rapid strengthening of the theoretical and engineering support be undertaken. Careful and prompt attention should be given to the building of a toroldal theta pinch (SCYLLAC). If the project is judged feasible, the design and fabrication of this facility should have high priority. However, in the opinion of the panel the success of this venture rests heavily on the presence of an adequate engineering staff and theoretical support which is currently lacking at LASL.

(7a) We find at the Lawrence Radiation Laboratory that: Three major lines of attack are making rapid progress and providing encouraging results. These are the minimum $B$ geometry mirror machine, Alice, employing neutral injection; the E-layer experiment, Astron; and the toroidal experiment, the Levitron. The work on Alice, along with supporting experiments in more modest mirror machines has played a leading role in reconciling stability theories with experiment and providing the scientific community with a large measure of hope for the ultimate understanding and exploitation of plasmas. The performance of Astron has been impressive to date. It is an ambitious engineering undertaking and has demonstrated that its E-layer ${ }^{*}$ is stable under conditions of modest field reversal.

The term E-layer refers to very energetic (relativistic) electrons which form a cylindrical shell. The role of this shell is 1) to help produce a magnetic field which can confine the plasmas and 2) to heat the plasma. 
Astron has no counterpart; it is the only unique method being pursued by this country. The series of experiments recently completed in the Levitron have clarified a number of significant points on the behavior of plasmas in toroidal configurations and pointed to several approaches which may ultimately circumvent the instabilities thwarting our efforts.

The large mirror experiment called $2 \mathrm{X}$ appears to be suffering from a succession of difficulties and its future prospects do not appear encouraging.

The supporting studies in basic aspects of plasma physics and theory at both the Livermore and Berkeley sites are of high quality.

(7b) We recommend that:

The Allce experiment utilizing neutral injection into a minimum B mirror machine be pushed vigorously toward the large-diameter short-length geometry favored by current understanding. Simultaneously a concerted effort should be made to develop and use improved injection sources having the required spread in energy and momentum as indicated by current theories. The minimum-B geometry produced by the baseball-seam coll is most interesting and this line of attack should be pursued energetically. Superconducting and/or cryogenlcally cooled colls should be developed and used to conserve power when such use is judged fearible. 
The minimum average $B$ studies in toroidal geometry employing the Levitron and its modification should be pursued rapidly in order to lay the groundwork for the next generation of stable toroidal confinement experiments. Proposals for new major facilities resulting from this program should receive early and careful attention. Coordination with Princeton proposals on toroidal experiments will be required to assure most effective utilization of all facilities.

The Astron experiment should be continued at a vigorous pace with careful attention given to significant bench marks lest the program be allowed either to drag or to outreach itself. Early and careful attention should be given to funding the proposed improved $4 \mathrm{MeV}$ accelerator. Construction of this facility should proceed quick1y once it is clear that E-layer stability can be maintained under conditions set forth in the present program. It is important to explore the full regime of E-layer stability and the ultimate potential of this approach to the CTR problem.

The theoretical investigations in direct support of Alice and the Levitron should be maintained at their present high level. The theoretical support of Astron should be increased. The link maintained between the Livermore and Berkeley sites is commendable in that it provides a valuable contact between the CTR professional staff and 
the teaching staff at the University of California, Berkeley. Every effort should be made to strengthen this link. The University should be encouraged to make more extensive use of the Berkeley facilities in their teaching and preparation of students in plasma physics. Similar recommendations apply to the ties between Livermore and the Davis campus. 


\section{CTR RESEARCH AT NON-AEC LABORATORIES}

(8a) We find that:

The programs at the Naval Research Laboratory, at General Atomic, and at General Electric are all of high professional quality. The program at Aerojet-General Nucleonics is still in its infancy but appears to be well conceived and offers promise. The interaction between these programs and the more extensive AEC supported effort has been valuable and is to be commended. In particular, the theta pinch programs at NRL and GE have had significant influence on the Los Alamos effort. The development of novel neutral injection sources at AGN is being followed closely by workers at Livermore. The theoretical effort at GA has played an important role in CTR both nationally and internationally.

(8b) We recommend that:

The AEC continue to maintain friendly and active contact between its program and allied efforts outside its immediate cognizance. These provide valuable additions to the total effort and contribute to the overall health of CTR research. However, the AEC can not afford to rely on outside sources to carry the burden of research in this difficult and time consuming field. The AEC must strive to set the pace and capitalize on the good fortune of having active collaborators. 


\section{U.S. AND WORLD PROGRAMS}

(9a) We find that:

World-wide progress toward the understanding of plasmas is now rapid. The problem of heating plasmas is now well on the way to being solved. Considerable progress has been made toward solving the much more difficult problem of plasma confinement. Here the stumbling block is the variety of instabilities peculiar to the plasma state. Many dangerous ones have already been conquered in open-ended systems. The list of remaining instabilities is long and still growing, but at a much diminished rate. Prospects are excellent that with time and sufficient attention these will be conquered as well. Our optimism rests on the fact that our ability to study plasmas has greatly improved, great strides have been made in the theoretical understanding of plasmas, and much progress is evident in the quantitative correspondence between theoretical predictions and experimental observations. In this the rest of the world has contributed as effectively as this nation.

We estimate the U.S. participa'ion in CTR research at one-fifth of the total world effort in terms of weighted expenditures and personnel. We further estimate that the effective contribution of this nation to the continuing progress is approximately onethird of the total. This is to be contrasted with the situation as it appeared four years ago when the U.S. effort represented 
nearly one half and its contribution amounted to well over onehalf the total. We conclude that the U.S. proportion of the total contribution is declining rapidly.

We note that during this four year period the AEC budget for CTR research has been essentially static and largely inflexible. This has resulted in a severe curtailment of the normal influx of new people with fresh ideas and new approaches. In this respect the panel notes the preponderance of youthful U.S.S.R. delegates at the recent international conference on CTR.

Another result of recent fiscal policies has been the lack in both speed and flexibility of adapting or acquiring equipment to test new ideas originated here and abroad. There has been a tendency to continue studies with equipment that is becoming outdated in an attempt to obtain maximum return from prior investment.

By contrast, the efforts in Western Germany, France, the U.K. and U.S.S.R. have expanded rapidly during this intervening period with new devices, new facilities, and vigorous youthful staff. During the same period intensive programs were created in Japan, Italy, and a number of other countries. 
(9b) We recommend that:

This country continue to play a leading role in controlled thermonuclear research. It can not afford to become second to any other nation in a field of such vital significance. By sharing in the world-wide results, the U.S. has advanced far beyond the stage it would have reached with only its own resources. In order to share and exploit future results, the U.S. program must continue strong and viable.

To this purpose the AEC should adopt and promote a fiscal policy which will implement the recommendations of this report and will attract talented young scientists and engineers into the CTR program under AEC auspices. In our opinion this will require a doubling of scientists and engineers engaged in CTR under AEC auspices in a period of approximately 5 years. 
(10a) We find that:

Work at the major AEC supported university plasma laboratories is yielding excellent returns in plasma physics and plasma diagnostic techniques for funds expended. The programs at Berkeley, at MIT, at Wisconsin and at New York University which were viewed by panel members appeared to be especially fruitful, with excellence in output of research results and of gifted plasma scientists and engineers. Work at Stanford and at the Stevens Institute of Technology is contributing to our understanding of plasma physics.

(10b) We recommend that:

The AEC recognize the critical role of universities in the training of plasma scientists and engineers needed to invigorate the CTR program in the nation. By increase of support to existing centers of good productivity and promise and by careful selection of new university centers, the AEC should promote expansion of these activities at a more rapid rate than in the programs of the major laboratories. This policy will help to provide the youthful talent needed at the major laboratories. AEC support at universities should extend to basic plasma studies, recognizing that thorough understanding will be required for achievement of the ultimate objective, and recognizing that students broadly trained in fundamentals are required for long time productivity in a rapidly developing field. The AEC should recognize that continuity of support and long time support are required for successful university programs. 
FUTURE CTR PROGRAM REQUIREMENTS

(11a) We find that:

The CTR program in this nation is declining in stature relative to that of the rest of the world. It will deteriorate rapidly to a secondary role if the present static budget of the AEC is continued. Only bold and imaginative measures will reverse this trend.

During the early period of CTR investigations this country held a commanding position. In the development of new machines, in the development of diagnostic equipment and refined measurement techniques, in the use of this equipment and these techniques for careful measurements of plasma properties this country made the major contributions.

Largely because of our work understanding of plasma properties is immensely superior to the situation ten years ago. Much remains to be done but the groundwork has been laid and we believe it has been laid solidly.

Other nations are now coming rapidly into the field with vigorous young talented workers. The Soviet and British efforts which began at the same times as ours are now fresh and rapidly evolving programs as a result of expansion and re-direction. Unless we take bold action we shall fare poorly in the competition. After carrying through with the difficult groundwork and making major contributions to the foundation we shall be in a relatively poor position to reap rewards as they come. 
1b) We recommend that:

The AEC take immediate steps toward establishing a national center for plasma studies and nuclear fusion research. This facility is to extend and stimulate the program in this country. It should serve as a major center for national and international exchange in the effort leading toward the development of controlled thermonuclear reactors.

The national center should have an identity of its own. It must be free of all security restrictions in order to engage in cooperative ventures with other nations conducting CTR programs. It should have close links to other CTR laboratories maintained by frequent visits and by exchange of personnel for periods of one to two years. The AEC is encouraged to promote exchange programs both on a wide domestic basis as well as internationally.

The national center should have a program broadly based in the fundamental aspects of plasma physics. It should have close ties to one or more universities and should play an important role in the teaching and preparation of students for careers in CTR. 
The program of the national center must aim for competence in a variety of containment devices. As the CTR program evolves, the need for new, larger volume containment devices is expected. The national center should play a principal role in implementing plans for large containment devices.

Recruitment of personnel should be started quickly on a scale sufficient to invigorate present AEC programs and to provide an experienced cadre upon which the national center can draw. 
Acknowledgement

Dr. Samuel King Allison led this panel during the most important period or its work, when information was being gathered. The final information gathering trip was to Culham England to attend the Second International Conference on Plasma Physics and Controlled Thermonuclear Research. During this conference Dr. Allison suddenly became ill. Eight days later he died. Members of the panel became deeply impressed with Dr. Allison during their service under his leadership. To his understanding, his wisdom and his tireless efforts much credit must go for the completion of the assignment.

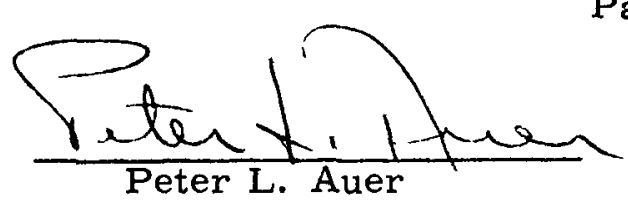

Peter L. Auer

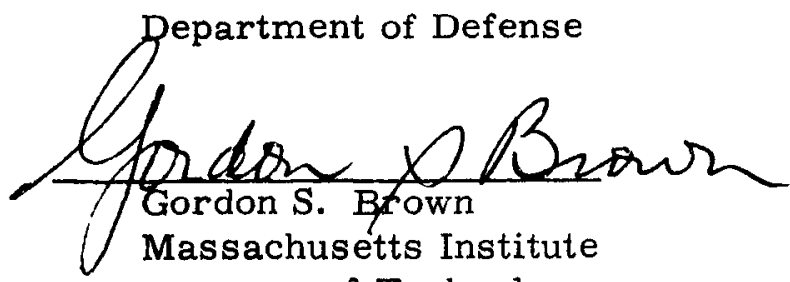
of Technology

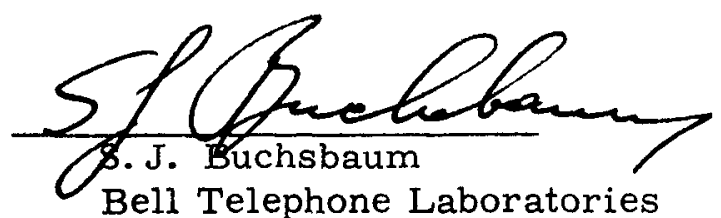

R.G. Herb, Chairman

University of Wisconsin

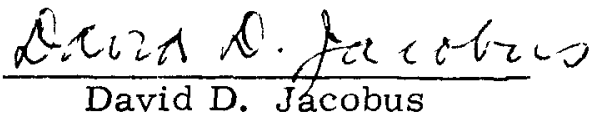

Harvard University

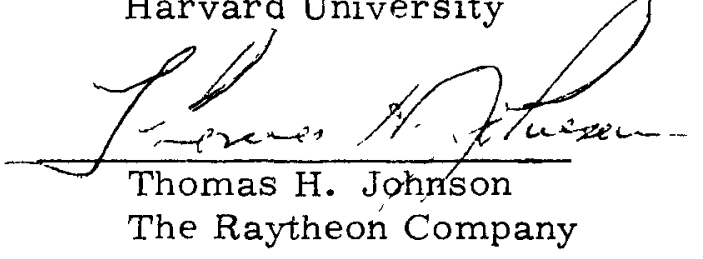

$\varnothing$<smiles>[C-]1=CC=CC1</smiles> 
Appendix 3

\section{Summary of Programs in AEC Laboratories}

\section{Plasma Physics Laboratory, Princeton University}

The controlled thermonuclear program at Princeton has for its ultimate goal the confinement of a deuterium-tritium plasma in a toroidal magnetic field under conditions appropriate for a fusion reactor. Typically this would involve containment for about one second at a density of $10^{15}$ particles per cubic centimeter and at a temperature of $10^{8}{ }^{\circ} \mathrm{C}$. The advantage of the toroidal geometry lies in the fact that it eliminates end losses, which are a potentially serious problem in all open-ended devices.

The major parts of the Princeton program to be described here are: 1) the investigation of toroidal equilibrium, 2) radiation and impurity problems, 3) plasma confinement experiments, 4) theoretical studies of instabilities as they pertain to plasma confinement, 5) plasma heating methods, 6) the program of fundamental plasma studies, theoretical and experimental, and finally, 7) summary of the program planned for the immediate future.

\section{1) Toroidal Equilibrium}

A plasma is said to be confined in equilibrium by a magnetic field when the net force tending to move the plasma toward the walls is zero. As Fermi pointed out many years ago, a simple torus with magnetic field everywhere parallel to the axis cannot confine a plasma in equilibrium. The difficulty is that the magnetic field is stronger near the inner radius and relatively weak near the outer radius. As a result, the positively and negatively charged particles drift in opposite directions, setting up electric fields perpendicular to the magnetic field. In the presence of crossed electric and magnetic fields, particles of both signs experience forces which drive them rapidly outward.

The stellarator concept of equilibrium meets this problem by twisting the magnetic field lines in such a manner as to provide direct paths around the torus, leading from regions where negative charges accumulate to regions where positive charges are in excess. The electrons, being highly mobile, move along the lines of force, continuously redistributing themselves in such a manner as virtually to eliminate the electric field caused by the drift.

The early stellarator idea produced the twisting of the magnetic field lines by a distortion of the entire configuration into the shape of a figure 8. For more recent models, a race track geometry has been 
adopted in which the twisting is accomplished by means of additional external helical coils.

The existence of this equilibrium, which eliminates the outward force, was verified experimentally. It was also shown that instead of eliminating the outward force, one can impose an additional inward force such that the two balance each other. This may be accomplished in several ways: (1) a current may be induced around the torus which interacts with an applied transverse field, or (2) a current may be allowed to flow across the discharge tube, which interacts with the toroidal field producing an inward force. These two latter methods are not as useful since the first can only be utilized in a pulsed device (the stellarator, in principle, may be operated in a continuous fashion) and the second requires appreciable plasma density at the wall or at collecting electrodes, which must be avoided if high temperatures are to be attained.

Several other types of toroidal equilibrium have been invented the bumpy torus, levitron, multipole, etc. The anticipated program of investigations of some of these types of equilibrium will be discussed.

\section{2) Radiation and Impurity Problems}

It was initially envisaged that the plasma creation and heating would be accomplished in several stages. In the first stage the plasma would be created and heated to about a million degrees by inducing an electric field around the torus, causing the cold neutral gas to become ionized and heated by the resultant current - i.e., ohmic heating. Experiments soon indicated that the formation of the plasma was accompanied by such a large influx of cold gas from the wall - mainly oxygen and carbon impurities - that the impurities completely dominated the discharge, masking the nature of the fundamental plasma processes taking place and strongly cooling the plasma by the resultant radiative energy losses.

Radiation is a very important factor with respect to fusion reactors. In a fully ionized plasma there can be only the continuous spectrum known as bremsstrahlung which results from the acceleration of free electrons in the field of the ions. However, even the cleanest plasmas contain some impurity atoms which are not completely stripped of bound electrons and the usual optical line radiation is therefore also important.

The radiated power, though small compared with black-body radiation, is by no means negligible. For thermonuclear reactors, the brems strahlung radiation from a pure hydrogen plasma sets an absolute powe $r$ limit to the operating temperature and a small percentage of heavy 
impurity atoms raises this limit enormously since the radiation rate increases as the square of the impurity ion charge $Z$. At thermonuclear temperatures the line radiation from impurities is small compared to the bremsstrahlung, but to get to high temperatures we usually start with low temperatures. Here ordinary radiative de-excitation can be seriously high. For example, a $25-\mathrm{eV}$ hydrogen plasma having a density of $10^{16} \mathrm{~cm}^{-3}$ with one percent fivefold ionized oxygen as an impurity radiates $4 \times 10^{4}$ watts $/ \mathrm{cm}^{3}$. This is $10^{4}$ times the brems strahlung rate and is clearly significant power loss, which if not made up would cause an e-fold drop in temperature in a few microseconds. In experimental plasmas even hydrogen can be responsible for appreciable radiation if ions lost to the walls are neutralized and return to the plas ma. In this "recycling" process excitation may absorb a large amount of energy.

As has already been stated, the discharge process in the early stellarator experiments was marked by an influx of impurities so large that the available power could not maintain the temperature. To improve this situation a major program was begun at Princeton to extend the newly-developed ultrahigh vacuum techniques of Alpert and his coworkers at Westinghouse to systems hundreds of times larger. The resulting baked, hard-seal vacuum systems produced base pressures below $5 \times 10^{-10}$ torr, an improvement of four orders of magnitude. This improvement resulted in more reproducible data, higher ion and electron temperatures, and a reduction in impurities of two orders of magnitude. Furthermore, a type of operation using low power and high repetition rate, termed discharge cleaning, which had previously been ineffective, now provided still cleaner discharges. The cleanest stellarator plasmas showed a maximum impurity concentration during the discharge of $5 \%$, with oxygen the main impurity.

The second technique used to control impurities was the divertor concept first described in the original stellarator proposal in 1951. Some early applications were also reported in 1958. The theory is that the plasma particles diffusing into the region near the wall are continuously guided by especially shaped magnetic fields into an outer chamber, or divertor, which is located at midpoint on one of the straight sections of the race track shaped tube. Hence most of the wall bombardment occurs in the divertor; gas streaming off the divertor wall cannot readily return into the discharge.

If a stellarator discharge is created under conditions such that the gas is at nearly uniform pressure, the divertor will also initially be filled with cold gas. This gas will flow steadily into the discharge tube during the pulse. To avoid this flow a pulsed gas feed is used. A 
pulsed valve admits a short burst of gas into the machine on the opposite side of the toroid from the divertor. Some 10 to 15 milliseconds later, when gas first starts to arrive at the divertor, the discharge is initiated. Since the pressure of cold gas in the divertor at this time is orders of magnitude below its mean value in the discharge tube, the influx of cold gas from the divertor is substantially reduced by this technique.

The divertor has been an outstanding success. With its use the impurity level during the discharge fell to 0.05 percent, a one-hundredfold reduction. A detailed study of the energy balance showed that the fraction of radiated power emanating from oxygen in a helium discharge dropped from $40 \%$ to less than $1 \%$. The combination of the divertor and pulsed gas feed reduced recycling in helium discharges a full order of magnitude.

\section{3) Plasma Confinement Experiments}

This improvement in impurity content not only removed a large energy drain but it also resulted in greatly improved reproducibility of experimental data, making possible a detailed study of plasma containment. With the development of microwave phase shift techniques it was possible to measure the change in plasma density with time resulting from plasma loss processes; however, this was partially masked by neutral gas reentering the plasma and being ionized. By means of absolute ultraviolet measurements, it was possible to measure the flux of returning hydrogen and thus find the true rate of plasma loss to the walls.

For magnetic field strengths ranging from 9 to 36 kilogauss, electron temperatures between 10 and $60 \mathrm{eV}$, and at densities varying from $5 \times 10^{12}$ to $3 \times 10^{13} \mathrm{~cm}^{-3}$, the se careful measurements showed that the confinement time is closely approximated by the formula

$$
\tau_{\mathrm{c}}=\pi \mathrm{r}_{\mathrm{o}}^{2} \frac{\mathrm{eB}}{\mathrm{ckT}} \text { seconds }
$$

where $r_{0}$ is the plasma radius and all other quantities are in cgs units. The plasma radius variation was rather limited; from 4 to $6 \mathrm{~cm}$. The results showed no explicit dependence of confinement time on ohmic heating current in the range 1.5 to 8 kiloamperes, nor on electron density.

In a second major group of experiments, the containment times were measured for a variety of different methods of plasma heating and even included the case of no heating whatever (i.e., after the heating mechanism was turned off). To allow for this latter possibility, the entire series of experiments was carried out at low plasma densities, in the range $5 \times 10^{9}$ to $5 \times 10^{12} \mathrm{~cm}^{-3}$ and in the electron temperature range of 0.5 to $4 \mathrm{eV}$. The great majority of this work was done at 12 kilogauss, 
but some measurements were made at 35 kilogauss. In this work the reproducibility was not as good as for the high-density work, but within a factor of two the confinement time was found to agree with the above formula for four operating conditions: 1) ohmic heating, 2) electron cyclotron heating, 3) resistive microwave heating, 4) no heating at all.

These measurements have made it possible to eliminate some of the theoretically possible mechanisms for anomalous particle loss (for example, the heating current itself could cause instabilities) and, correspondingly, to concentrate both theoretical and experimental work on assessment of the remaining candidates (or to invent new ones).

\section{4) Instability Theory}

One of the major outstanding theoretical questions for the whole Sherwood program is the understanding of anomalous particle and energy losses. It is the present belief of most workers in the field that instabilities are basically responsible for the anomalous loss in toroidal systems since they create fluctuating fields which may grow to large amplitudes (such that the applicable theoretical equations are nonlinear) and cause drifts across the magnetic field lines. Since the current theoretical understanding of the nonlinear domain of behavior is severely limited, so that it is not at present possible to decide which instabilities are harmless, it has seemed most reasonable to try to "curb" the anomalous losses by finding the conditions necessary to prevent the instabilities. Estimates of anomalous diffusion based on thermodynamic considerations indicate that the most dangerous modes in toroidal devices are those of low frequency and long wavelength compared to the ion cyclotron frequency and the ion larmor radius. Thus, there has been a major effort, both in this Laboratory and others all over the world, to examine the nature of these modes and ways of stabilizing them.

Most of the theoretical work has concentrated on low- $\beta$ plasmas since these are currently of most experimental interest. In the low- $\beta$ domain it has recently been demonstrated that there are only two basic modes which can arise. It has thus been found possible to classify the huge volume of literature on low-frequency instabilities by showing that the results fall in one category or another, with an occasional slight mixing of the modes in a particular regime of parameters.

A twisted and sheared magnetic field structure was originally proposed to overcome some versions of the hydrodynamic or flute mode. More recently, a great deal of attention has been focussed on minimum average $B$ ( $\min \bar{B}$ ) systems in which the magnetic field increases outward on the average. The reason for this interest is that such fields stabilize other versions of the flute mode in which dissipation plays a role. 
In Tables $I$ and $I I$ we have given the current theoretical picture of the various versions of these modes and their stabilizing mechanisms. We can then ask (1) which of these modes should exist in present stellarators, and (2) which can be stabilized without changing the present stellarator magnetic configuration. The answer to ( 1 ) is that as far as the hydromagnetic mode is concerned, both the resistive $g$ and the tear ing versions are unstable and all four versions of the drift mode are unstable. However, if a temperature of the order of $200-300 \mathrm{eV}$ could be achieved at densities of $10^{12}$ particles per $\mathrm{cm}^{3}$, both the short wavelength resistive $g$ and the tearing modes should be stabilized.

According to our best theoretical estimates at present, however, we do not have enough shear in the present stellarator to stabilize the drift modes. It is also worth noting that the extensive set of double probe measurements made on the Etude device show a spatial structure and frequency which is in rough agreement with the theoretical predictions.

The conclusions to be drawn for future devices appear to be optimistic in that with an appropriate combination of shear, min $\bar{B}$, and high enough temperature, all these low-frequency modes can be stabilized. It should be emphasized, however, that at the present time precise numerical stability criteria are lacking, and thus much work remains to be done.

\section{5) Plasma Heating Methods}

To obtain a hot plasma in a magnetic field it is possible to proceed along any of three basically different lines: (1) an already heated plasma may be injected into the magnetic field and trapped there; (2) energetic particles may be injected into the magnetic field and allowed to build up gradually to densities of interest; or (3) a cold gas may be admitted to the magnetic field region and there ionized, trapped, and heated.

The first two methods have been made the subject of small-scale investigations at Princeton. The results in the case of hot plasma injection indicate that the trapping in the stellarator configuration would be quite inefficient. This approach has therefore been abandoned. Energetic neutral injection looks promising provided that trapping efficiency can be improved by an order of magnitude and that sources can be developed giving a flux about ten times that of existing sources. Developmental work along these lines is now in progress.

The third method - that of ionizing and heating in the presence of a confining magnetic field - has been employed in almost all of the 


\section{Table I}

\begin{tabular}{c|c|c}
$\begin{array}{c}\text { Hydromagnetic or } \\
\text { Interchange Modes }\end{array}$ & Characteristics & Stabilization Mechanism \\
\hline $\begin{array}{c}\text { Hydromagnetic inter - } \\
\text { change or flute }\end{array}$ & $\begin{array}{c}\text { As sumes dissipation } \\
\text { negligible requiring high } \\
\text { temperatures }\end{array}$ & Shear and/or min $\overline{\mathrm{B}}$ \\
\hline $\begin{array}{c}\text { Hydromagnetic balloon- } \\
\text { ing }\end{array}$ & $\begin{array}{c}\text { Same as above with high } \beta \\
\text { Resistive g }\end{array}$ & $\begin{array}{c}\text { Shear and/or min } \overline{\mathrm{B}} \\
+\beta<\beta \text { critical }\end{array}$ \\
\hline $\begin{array}{c}\text { An interchange with dis - } \\
\text { sipation included }\end{array}$ & $\begin{array}{l}\text { Shear and/or min } \overline{\mathrm{B}} \text { and } \\
\text { high enough temperature } \\
\text { for viscous stabilization } \\
\text { of short wavelengths }\end{array}$ \\
\hline Tearing & $\begin{array}{c}\text { Same as resistive } \\
\text { broad spatially }\end{array}$ & $\begin{array}{l}\text { Shear and/or min } \overline{\mathrm{B}} \text { and } \\
\text { finite larmor radius } \\
\text { stabilization at moderate } \\
\text { temperatures }\end{array}$
\end{tabular}

\section{Table II}

\begin{tabular}{c|c|c} 
Drift Modes & Characteristics & Stabilization Mechanism \\
\hline Universal & $\begin{array}{c}\text { No collisional dissipation } \\
\text { and thus high temperature }\end{array}$ & Shear \\
\hline Resistive drift & $\begin{array}{c}\text { Almost the same as the } \\
\text { universal but includes } \\
\text { dissipation }\end{array}$ & Shear \\
\hline Resistive ballooning & $\begin{array}{c}\text { Same as resistive drift } \\
\text { but occurs in min } \bar{B} \\
\text { systems }\end{array}$ & Shear
\end{tabular}


Princeton controlled thermonuclear research experiments to date. Typically the working gas, which may be deuterium but is more commonly helium or hydrogen, is admitted into a toroidal vacuum magnetic field at sufficient pressure to yield a plasma density of the order of $10^{13}$ particles per cubic centimeter. Preionization of a small fraction of the gas is achieved by means of $x$ rays and, in some cases, the application of an rf voltage across a ceramic section in the otherwise stainless steel vacuum chamber. More powerful methods of energy coupling, such as 1) ohmic heating, 2) ion cyclotron heating, or 3) magnetic pumping, may then be applied.

1) Ohmic Heating. In the ohmic heating of a toroidal discharge an induced voltage is applied around the toroid. A current is produced which ionizes remaining neutrals and at the same time heats by collisional processes. In a stellarator this current flows predominantly parallel to the confining magnetic field. The heating effect of the current is proportional to the plasma resistivity, $\eta$. Since $\eta$ varies as $\mathrm{T}^{-3 / 2}$ and excessive currents result in instabilities, the effectiveness of ohmic heating decreases with increasing $T$. As a result, ohmic heating is an effective means for heating a plasma only up to about $1,000,000^{\circ}$ or about $100 \mathrm{eV}$.

2) Ion Cyclotron Resonance Heating (ICRH). To proceed to higher temperature a different concept is used: waves are set up in the plasma, and the wave energy is subsequently converted to thermal energy of the ions. One method for accomplishing this, first pointed out in 1958, is the inductive (i.e., electrodeless) coupling of energy into ion cyclotron waves. Such coupling should have an efficiency $>50 \%$.

The discussion of the generation and transmission of these waves is facilitated by introduction of the parameter $\Omega \equiv \omega / \omega_{\mathrm{ci}}$, where $\omega$ is the frequency of the generated wave and $\omega_{\mathrm{ci}}$ is the ion cyclotron fre quency. For $\Omega<1$, the waves can be efficiently generated in the plasma and are transmitted with little attenuation. If the waves enter a region in which $\Omega \rightarrow 1$ (called a magnetic beach), the wavelength goes to zero and the wave energy is transferred to the ions. (The situation is analogous to the manner in which the energy in water waves is converted to thermal energy as the waves roll onto a shallow beach.)

Experiments with ICRH in the B-65 and B-66 devices have borne out the theoretical predictions of wave formation and absorption. A high efficiency of coupling the input $\mathrm{rf}$ energy to the plasma exists, typically $50 \%$. The waves launched in the plasma exhibit the predicted properties of the ion cyclotron wave, and are absorbed with similarly high efficiencies when a magnetic beach is reached. Near the point of wave absorption, ion temperatures of $\sim 10^{\circ} \mathrm{K}$ have been measured. 
ICRH has also been applied to the $C$ stellarator operating in a number of different modes: (1) under conditions where the energy is deposited in the beach and is then allowed to flow out into most of the volume, ICRH resulted in an increase in ion temperature by about a factor of 5, giving a temperature of about 2 million degrees; (2) under conditions where the energy was deposited in a mirror section of the torus, the deuterium ion temperature rose to about 20 million degrees; and (3) when conditions were arranged to deposit the energy in the deuterium ions of a mixture of deuterium and hydrogen ( 1 to 9 ratio), deuterium ion temperature of 100 million degrees was reached.

3) Magnetic Pumping. Following ohmic heating, magnetic pumping was initially proposed as the method to be used to get the plasma up to thermonuclear temperatures. It involves the superposition of an oscillating magnetic field at about $100 \mathrm{kc}$, parallel to the main (static) magnetic field. The resultant magnetic lines of force and the plasma move in and out - hence the name "magnetic pumping." This configuration was proposed as a way to heat primarily the ions in a plasma.

The cyclical pumping of an adiabatic gas does not result in any heating. To achieve plasma heating the pumping frequency is chosen to emphasize some process which introduces a phase lag between density and temperature. In "collisional magnetic pumping" the pumping frequency is made about equal to the ion-ion collision frequency, and calculations show that this process can heat ions to a high temperature. The frequencies required are quite high, thus involving excessive voltages. When magnetic pumping is introduced in a short section of a stellarator plasma, one may, as a second possibility, adjust the pumping period to correspond approximately to the transit time of an acoustic wave, or, equivalently, to the transit time of an ion moving with thermal speed.

If the pumping frequency tracks its optimum value during the rise of plasma temperature, the efficiency of heating rises as $\mathrm{nT}^{3 / 2}$. Magnetic pumping is, in principle, fully adaptable to large machines, and for a high-density, high-temperature, fusion-reactor plasma.

This heating method is, however, a fairly slow one initially, and would not be useful with the loss rates presently encountered. Thus, this method is being held in abeyance until such time as a more stable plasma is achieved.

\section{6) a. Fundamental Theoretical Research}

Although the two areas of major theoretical interest at Princeton are the equilibrium and stability of plasmas confined in toroidal 
magnetic fields, it has frequently proven desirable, and indeed necessary, to pursue problems of a more basic nature. A few examples may be cited:

(1) Kinetic Theory of Plasmas. One extremely important question is what equations correctly describe a plasma. The FokkerPlanck equation as applied to coulomb collisions by Landau introduces a cutoff in impact parameter at the Debye length. By following the method of Bogoliubov, it was found possible here, in 1959, to derive a kinetic equation without an arbitrary cutoff. This equation gives Landau's result to a satisfactory approximation in many cases, and thus establishes a sound theoretical basis for treating a plasma by the kinetic equation. The important cases in which the above work does not apply e.g., plasmas with velocity space instabilities - have also been studied at this Laboratory. The two-body correlation function is an important element in this theory and its relation to emission and scattering of radiation gives an added importance to these researches. In order to solve these problems, a "two time-scale" approach has been developed which has important applications to areas of kinetic theory other than plasmas. Further research will be continued on these fundamental problems which are basic to any true understanding of plasmas.

(2) Adiabatic Invariants and Charged Particle Motions in Electromagnetic Fields. In order to determine the containment of a charged particle in any device over a long period of time it is necessary to know constants for the motion, since it is manifestly impossible to follow the detailed orbit. Such constants, the adiabatic invariants, must be valid to a much greater accuracy than the lowest order to which they are usually known. From calculations made here in 1957 it was shown that one of the adiabatic invariants, the magnetic moment, is constant to all orders (in an asymptotic sense) in the smallness of the gyration radius as an expansion parameter. Therefore, it was possible to develop a practical scheme for following a particle in time over long periods. This was a generalization of the guiding center theory. From this work there later developed, in 1961, an elegant and general extension of this earlier work to all Hamiltonian systems with a similar expansion parameter. This theory not only gave all the adiabatic invariants of a particle, but proved also to be of wide interest in all of mechanics. The results of this general theory are still being explored.

(3) Plasma Conductivity and Radiation. Extensive studies have also been made of the high-frequency conductivity of a plasma. From work mentioned previously, it is found that the Fokker-Planck equation cannot be used for this purpose and that this problem involves advanced kinetic theory. Results from the above studies give the absorption of electromagnetic radiation, and by detailed balance the emission 
of radiation in the microwave region where plasma effects (such as twobody correlations and dispersion effects) are important. Furthermore, a direct method has been developed for calculating radiation by a test particle approach which gives in an unambiguous fashion such phenomena as longitudinal wave-transverse wave interactions in wave-wave scattering, synchrotron emission, and ordinary bremsstrahlung.

(4) One-Dimensional Models of a Plasma. Many areas of plasma physics such as nonlinear effects and kinetic theory, which are too complicated to be treated analytically, can be treated numerically by a one-dimensional model of a plasma developed at this Laboratory in 1960. Important problems such as the nonlinear limit of the twostream instability and the relaxation rate by fine-scale mixing have been investigated on this model. In conjunction with the se numerical experiments, the statistical mechanics of one-dimensional plasmas has been developed here. The model has recently been extended to take into account collisions. For example, interesting results are being found on the effect of collisions on the instability as sociated with a double-peaked velocity distribution function.

(5) Nonlinear Phenomena. One of the most difficult and important areas of plasma physics is nonlinear phenomena. Until these are understood one cannot predict the consequences of an instability the existence of which is known from linear theory. In addition to the work already mentioned, basic contributions have been made to other nonlinear oscillation problems. The quasi-linear theory has also been studied in detail. This area of research will undoubtedly be a principal one in the future.

6) b. Fundamental Experiments

While theory has made great strides, the physical situation is so complex that all theories are based on simplified models. Therefore, experiments designed to test the range of applicability of the se theories are essential to progress in plasma physics in general and to thermonuclear goals in particular. Much work of this sort has been accomplished; for the sake of simplicity, on linear devices. Thus the Q devices provide an especially simple medium for some of the se experiments. In this device a cesium vapor is ionized on contact with hot tungsten plates located at each end. The result is a seemingly quiescent column of plasma between the plates, available steady-state for the study of controlled disturbances and other plasma phenomena. Basic experimental plasma research at Princeton is presently carried out on two $Q$ devices (Q-1 and $Q-3)$, two mirror devices (ECR-1 and B-66), and, part time, on the stellarators (Etude, B-65, B-3, and C). Several examples of recent work will be described. 
(1) Instability Studies on the Etude Stellarator. Figure 1 is a representation of density disturbances of an ohmically-heated plasma in the Etude stellarator under kink-unstable conditions and was obtained with an array of Langmuir probes spaced around the column, in a plane perpendicular to the magnetic confining field. Simultaneous oscillograms of the ion currents to the probes gave the azimuthal variation of plasma density at successive instants of time; these reconstructed instantaneous configurations are shown on the figure at one microsecond intervals. The abscissa is the probe number, which goes from 1 to 24 , so that the probe-to-probe spacing is $15^{\circ}$ (which is $0.5 \mathrm{~cm}$ ). About $480^{\circ}$ of azimuth are shown for greater clarity. The fluctuation amplitude was normalized to the mean local density, and is scaled on the figure so that an amplitude equal to a one-microsecond ordinate interval is a $40 \%$ fluctuation in density.

The most apparent feature of the fluctuations is their large amplitude; also, the haphazard drift of the perturbation toward the left, which corresponds roughly with the $\mathrm{E} \times \mathrm{B}$ motion expected. The fourfold periodicity in the data displayed on the right-hand side of the figure corresponds to the value predicted for a kink mode if the actual column diameter is $5 \%$ smaller than the one as sumed. For the conditions of the figure at the left, one should expect an $\mathrm{m}=1$ symmetry.

Lastly, one notes that the perturbations appear to change on a time scale of a microsecond or so. One can either conclude that the perturbations grow at this rate, or else that "mature" perturbations move in and out radially at velocities of $10^{6} \mathrm{~cm} / \mathrm{sec}$.

(2) Instability Studies on the Q Devices. The most positive identification of instabilities has come from the cesium $Q$ machines. In two instances at this Laboratory the experiment preceded the theory. The first observation was the current-excitation of electrostatic ion cyclotron waves propagating radially across $\mathrm{B}$. Both the 'frequency $\omega \approx 1.2 \omega_{c i}$ and the critical current $j \approx 12$ nev $v_{i}$ thermal agreed well with theory. The second instance was the positive identification of the universal instability. This is an unstable electrostatic wave propagating almost purely in the azimuthal direction with a velocity nearly equal to the electron pressure-gradient drift velocity. The wavelength parallel to $\underset{\mathrm{B}}{\mathrm{B}}$ is extremely long. Both the frequency and wavelength are in good agreement with either the collisionless universal instability theories developed in the USSR or counterpart resistive theories developed at this Laboratory; the large resistivity in a cesium plasma suggests that it may well be the resistive drift wave which is observed. This instability is suppressed by conducting end plates, and its onset can be observed by varying the conductivity of the sheath at the end plates. The stability criterion has been computed here and is roughly 

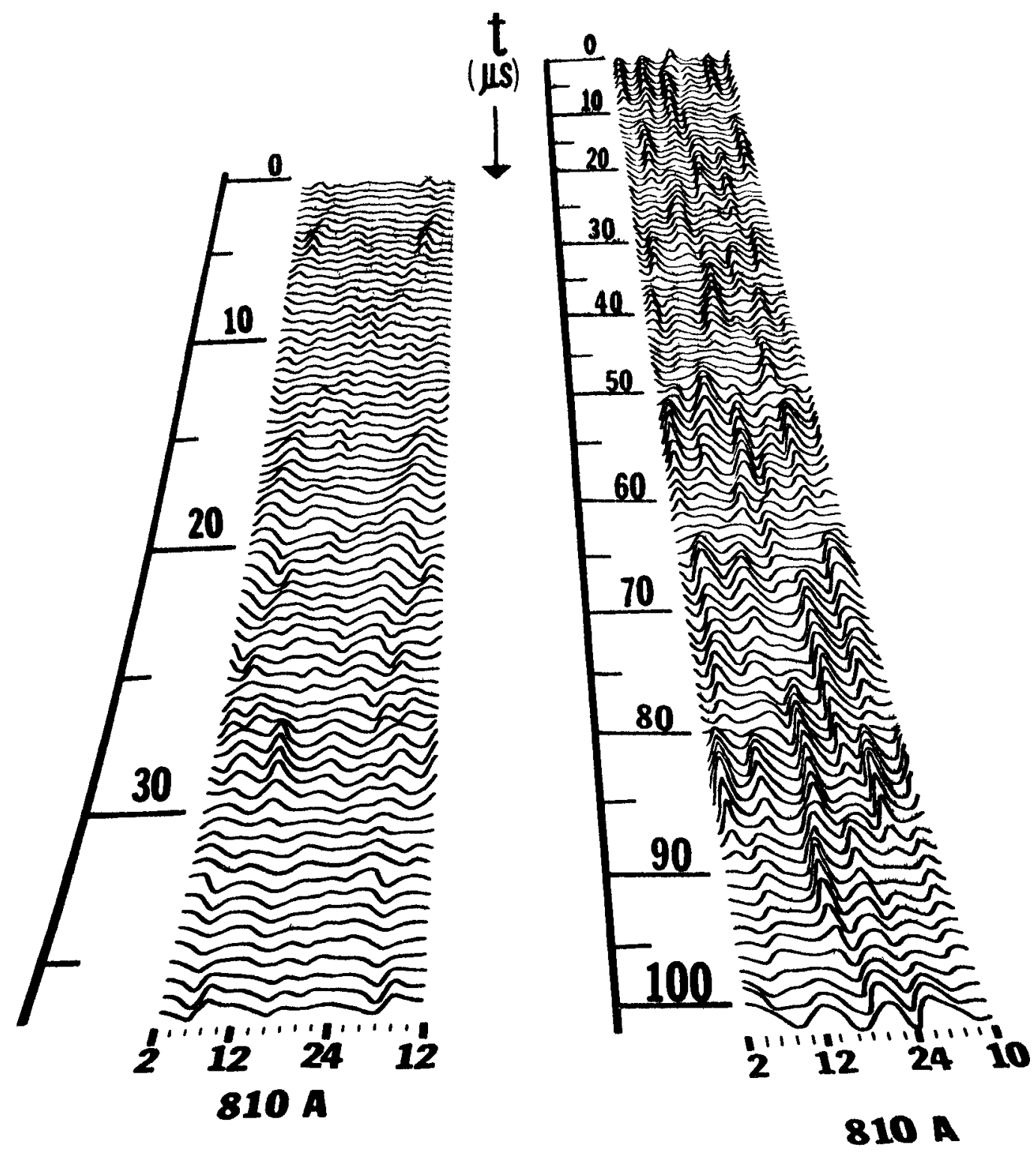

643547

Figure 1 
verified experimentally. Recently scientists here have studied the resistive $\mathrm{g}$ instability by gradually increasing the curvature of an initially uniform field containing a quiescent plasma. Oscillations of the proper velocity and wavelength are found to start at a critical radius of curvature roughly in agreement with theory. Furthermore, enhanced "diffusion" seems to occur with the oscillations. When a curvature is imposed, the initial equilibrium of the plasma is lost; but the experimental conditions can be chosen so that the loss of equilibrium is a negligible effect. It appears theoretically that no other curvature-dependent instability occurs in a highly resistive plasma in the absence of other driving forces.

(3) Electron Velocity Distribution in ECR-1. The ECR device is a small magnetic mirror with a 4-kilogauss central field. A 2 -liter plasma is produced by about $10 \mathrm{~kW}$ of electromagnetic radiation at $10 \mathrm{Gc} / \mathrm{sec}$, which is introduced axially through the mirrors. With the input microwave power applied, a 10 -gauss diamagnetic signal has been observed with an electron density reliably measured to be $5 \times 10^{11} \mathrm{~cm}^{-3}$. Upon removing the heating power, the diamagnetic signal and the density decay together with an e-folding time of about $2 \mathrm{msec}$. If all the electrons had similar energies, the density and diamagnetic signal would correspond to a temperature of several kilovolts.

To determine the energy distribution of the particles in the plasma a low-power microwave transmission experiment was performed at the second harmonic of the electron gyration frequency. It was found quite unambiguously that two components were present one at a very high energy and one very low. The cold part, which constitutes most of the electron density, has a temperature of less than $100 \mathrm{eV}$. The other ("runaway") component, which is responsible for essentially the whole diamagnetic signal, has an energy of more than $50 \mathrm{keV}$.

Changes intended to eliminate the causes of the runaway discharges are now in progress. They include increasing the mirror ratio, modulating the microwave frequency slightly, and increasing the microwave power.

(4) Absorption Near the Second Harmonic of the Electron Cyclotron Frequency in the C Stellarator. A particle moving uniformly through an electric field which is periodic in both space and time will feel the field at a frequency shifted by the Doppler effect. Although the analysis is somewhat more complex, a similar effect occurs for a particle gyrating in a spatially-periodic field. The particle now feels a number of component fields with frequencies given by the 
laboratory-frame wave frequency plus or minus the various integral harmonics of the gyration frequency. The relative strength of different harmonics depends on the ratio of gyration radius to wavelength. Using the Boltzmann-Vlasov equation to take finite-larmor-radius motions into account (the fluid equations are inadequate to reveal this effect), one finds that wave-energy absorption is expected in the vicinity of the cyclotron harmonic frequencies.

By transmitting across the uniform-magnetic-field section of the Model C stellarator, it was found possible to locate the second harmonic absorption resonance. For $90^{\circ}$ transmission, the full width of the peak at half maximum is only $0.05 \%$ - so narrow that special equipment had to be added to the $C$ control circuits to provide adequate reproducibility in the magnetic field pulses. With transmission at other-than-normal incidence, the resonance is Doppler-broadened. The shape of the broadened resonance line then reproduces, almost without correction, the shape of the electron velocity distribution for motion parallel to the magnetic field. The depth of the absorption line is proportional to the electron pressure perpendicular to the magnetic field. Quantitative agreement has been obtained between the se line shapes and measures of electron temperature by other means.

7) Program for the Immediate Future

\section{A. C Stellarator}

(1) Efforts will be continued to achieve a better scientific understanding of the nature of the processes giving use to anomalous losses in the $C$ stellarator. This involves a number of new techniques:

(a) A very rapidly opening diaphragm has been developed which will make it possible to directly observe the rate of plasma motion across the field - and the details of this motion.

(b) A method has been developed to determine experimentally whether lines of force, due to possible inexact coil placements, do wander out to the walls, thus providing a direct escape path.

(c) The fluctuating fields inside the plasma will be examined more thoroughly.

(2) Experiments aimed at greater ICRH effectiveness are planned; thus far the power applied has been severely limited by 
technological difficulties. An ingenious solution has been found which should result in about a fourfold increase in power.

(3) It may be possible to increase the shear in the magnetic field sufficiently to meet the (as yet inexact) theoretical conditions for stabilizing the drift modes.

\section{B. Other Toroidal Experiments}

(1) A continued intensive effort is planned, comparing the relative merits of the various proposed minimum $\bar{B}$ geometries on the basis of existing though incomplete stability theory.

(2) The above theoretical work is expected to make possible within a few months a decision to build a particular minimum $\bar{B}$ system. This will be a program of major importance to the Laboratory.

\section{Other Aspects}

(1) Since some of the drift modes have appeared in the relatively simple machines, the effectiveness of shear in suppressing at least the resistive shear mode is possible and is planned.

(2) Continued research is planned on the generation and damping of plasma waves. This work is of interest from many standpoints. It relates to both confinement and heating, and in addition is of interest from a fundamental scientific standpoint.

(3) The participation of graduate students, now numbering about 20 , will be increased by about $50 \%$ in the next two years.

(4) An enlarged theoretical effort involving students and visiting scientists will add new areas of theoretical competence particularly in nonlinear theory. 


\section{Oak Ridge National Laboratory.}

The features of the Oak Ridge vork that distinguish it from the work at other laboratories are: 1) a concentration upon steadystate systems, In contrast to the pulsed systems that are widely used elsewhere; 2) a long-time specialization on injectionaccumalation systems (DCX-1, DCX-2, and more recently INTEREM); 3) the pursuit of special subjects such as turbulent heating by beam-plasma interaction (the BURNOUT experinents) and electron cyclotron heating. Most of the experiments have so far been carried out in magnetic mirror geometry; most of them can later be adapted to magnetic wells of conventional type if present stabilization methods fail. There are at present no closed (toroidal) systems under investigation at Oak Riuge.

\section{$\underline{\mathrm{DCX}-1}$}

This series ot experiments has just drawn to a close, but the history has been distinguished and illuminating, and it is worth reviewing here.

The principle is 1llustrated in Fig. 1. A beam of $\mathrm{H}_{2}^{+}$ions is accelerated to $600 \mathrm{keV}$, and sent into the middle of a magnetic trap between mirror coils. Most of the beam simply loops around and re-emerges, but if for any reason some of the $\mathrm{H}_{2}^{+}$(molecular) ions are dissociated in the magnetic field, the resulting $\mathrm{II}^{+}$ (atomic) lons at $300 \mathrm{keV}$ will be trapped, and will orbit in the field until some loss mechanism releases them. Note that the ions are plenty hot enough for fusion (on eventual transfer from $\mathrm{H}_{2}^{+}$to $\mathrm{D}_{2}^{+}$or $\mathrm{DT}^{+}$); the objective is to build up the density of the accumulating atomic ions toward the maximum value that can be retalned in the magnetic field, $\forall i z$, about $10^{12}-10^{13}$ per cubic centimeter. 
The DCX-1 experiments passed through three phases. In the first, a vacuum carbon arc was run lengthwise through the apparatus, as shown in the diagram. Its purpose was to aid in the dissociation of the $\mathrm{H}_{2}^{+}$ions to $\mathrm{H}^{+}$. It did this well, and densities of $2 \times 10^{9}$ fast $\mathrm{H}^{+}$ions per cubic $\mathrm{cm}$ were reached, at which level the loss mechanisms were found to be such that the use of the arc was abandoned in favor of a second approach.

In the second approach the arc was omitted, and emphasis was placed upon a very clean system with an excellent vacuum. The purpose of this was to increase the mean residence time of trapped $\mathrm{H}^{+}$Lons, because calculation showed that if a threshold value of 300 milliampere-seconds could be surpassed in the product of the injected beam current (milliamperes) times the mean residence time (seconds), then the $\mathrm{H}_{2}{ }^{+}$ions would dissociate upon fast $\mathrm{H}^{+}$ions already trapped, and the plasma density would then exponentiate, that is, it would build up upon itself and autamatically rise of its own accord. By improvements in technique the mean residence time was greatly increased (to two minutes under some conditions), and the threshold was passed. No sign of exponentiation was found, and the reason was that the plasma spread both radially and longitudinally, thwarting the desired increase in density. Still, no direct loss of the trapped protons was observed; the sole loss mechanism depended upon the presence of the background gas.

It was then necessary to understand what caused the spreading, and for this purpose the third phase of experimentation was started. The trapping process was improved so that $\mathrm{H}^{+}$ions were trapped about ten times as fast as previously. This required the development and exploitation of a subtle process (invented but not developed elsewhere) called Lorentz dissociation, 
which we shall not describe here. When it was applied, it forced the revelation of an instability that limited the plasma density to $2 \times 10^{8}$ fast $\mathrm{H}^{+}$ions per cubic cm; at this level, $\mathrm{H}^{+}$lons are lost directly as fast as they are injected. An elegant interplay of theory and diagnosis then identified the instability as the negative mass instability, previously known in accelerator development.

Although the DCX-1 experiments have led to accumulated densities that are low when compared with our goal, nevertheless they have polnted the way to go. The negative mass instability can be overcome if the magnetic field is made to increase as one goes outward from the axis, instead of decreasing as it does in the conventional magnetic mirror arrangement. Fortunately this is a direction strongly favored for the control of other instabilities (see Section II H of this document). Also the DCX-1 experiments strongly support the theoretical contention that it is important to randomize the injected beam, particularly by introducing a spread in energy. Add to this recent theoretical recommendations that the plasma be many Larmor diameters across, and that it be rather short and fat in shape, and the next experiment emerges with clarity: injection of low-energy (say $5-7 \mathrm{keV}$ ) $\mathrm{H}^{\circ}$ atoms, with a mixture of energles, into a magnetic well. Preparations for this are under way. The new experiment will be called DCX-3.

Recent developments force us to add a final paragraph to the DCX-1 story. For some time we have been studying wall treatments as a means of plasma stabilization. The work has been done in a calutron, the semicircular beam of which is a rough model of the full-circular trapped $\mathrm{H}^{+}$ ions in DCX-1. Here it had been found that if the end walls inside the vacuum vessel are covered with separated plates, each of which is connected to ground through a small inductance, then the calutron beam was magically 
stabilized. A satisfactory theory for the effect has not yet been developed, but nevertheless the technique has been applied to DCX-1. The results so far have surprised and pleased us. At low densities all indications of the negative mass instability can unquestionably be squelched. The key experiment, however, has yet to be done; that is, to try to exceed the previous density limit at $2 \times 10^{8} \mathrm{H}^{+}$ions per cubic $\mathrm{cm}$, and to see how far one can go. This is now being done.

\section{$\underline{\mathrm{DCX}-2}$}

This experiment resembles $\mathrm{DCX}-1$ in that $600-\mathrm{keV} \mathrm{H}_{2}{ }^{+}$ions are injected and one seeks to accumulate the hot $\mathrm{H}^{+}$ions formed by dissociation. The arrangement is shown in Fig. 2. The $\mathrm{H}_{2}{ }^{+}$ions are injected deeply into the magnetic field near one mirror coil through a slanted, magnetically shielded snout. They clear the snout on their first turn and spiral to the far mirror (about 2 meters away) where they are reflected so that they spiral back toward the injection end. Most of the DCX-2 experiments have been done using a lithium vacuum arc (developed at Oak Ridge for the purpose) about 20 feet long running lengthwise through the apparatus. The spiralling $\mathrm{H}_{2}^{+}$ions pass through the arc 100 times or more, and nearly all of them are dissociated before they move back far enough to strike the snout.

Under these conditions, with an injected beam of about 40 ma of $\mathrm{H}_{2}^{+}$, densities of $5 \times 109$ fast $\mathrm{H}^{+}$ions per cubic $\mathrm{cm}$ have been reached in a volume as large as 150 litres.

The performance of DCX-2 is complex, and is still under intensive study. Four unpredictable and striking observations will be noted here:

1) Flutes do not develop; the losses so far seem to depend only upon background gas and the lithium arc; 
2) The $\mathrm{H}^{+}$ions, although trapped at $300 \mathrm{keV}$, quickly spread in energy. Some lose energy, and others gain. Those that gain energy live much longer vis-a-vis the loss processes that are present, and hence the fast-1on density quoted above pertains to a mean energy above the trapping energy. This is no small effect; mean energies of the contained fast ions have been measured at 3 or 4 times the trapping energy.

3) One would think that the $\mathrm{H}^{+}$lons would retain some of the longitudinal motion of the spiralling $\mathrm{H}_{2}{ }^{+}$ions from which they are derived, but this is not the case. The dominant fast $\mathrm{H}^{+}$ population has no longitudinal velocity (under an appropriate adjustment of the magnetic field), and this has the useful consequence that the contrined fast $\mathrm{H}^{+}$ions do not strike the snout.

4) Radiofrequency observations of the DCX-2 plasma reveal a great richness in the harmonics of the proton cyclotron frequency. Harmontcs up to the $100^{\text {th }}$ have been observed.

Present studies are directed mostly toward an increase of understanding of DCX-2. Efforts toward an increase of density include a) improving the vacuum; b) using a hydrogen arc instead of the lithium arc; $c$ ) increasing the $600-\mathrm{keV} \mathrm{H}_{2}{ }^{+}$beam; d) injecting $1 / 2$ ampere of $100 \mathrm{keV} \mathrm{H}{ }^{+}$ions into the end, just inside the mirror cone. All of these experiments have either been tried in a preliminary way, or are about to be tried. Meanwhile, the fact remains that $D C X-2$ contains the hottest plasma in captivity, and the densest plasme emong the class of experiments in which it belongs. 


\section{Electron Cyclotron Heating}

This is an oblique approach. A plasma is generated by an efficient means, a stable regime is found, and the plasma is then modified toward fusion. We have found that the first two objectives can be reached if one starts by heating the electrons rather than the ions.

The apparatus is diagramed in Fig. 3. Again we have a pair of mirror coils. Between them in a vacuum tank is a copper cavity with channeled ends. Vecuum pumps outside the mirror coils complete the picture. Microwave power is fed into the central cavity, and deuterium gas is introduced to a pressure of $10^{-5}$ torr. The magnetic field strength is then adjusted until the condition for electron cyclotron resonance is met in zones near the ends of the cavity. When this is done plasma is abundantly generated as the gas absorbs the microwave power. A great flood of $x$ rays testifies to the efriciency of the electron heating, cold plasma streams to the regions of the pumps, und when a proper balance of gas feed and microwave power is found, a stable regime can be established.

Taking the experiment called EPA as an example, we have found that plasma has the following steady-state characteristics when heated with $30 \mathrm{~kW}$ of 3-cm microwave power:

Density $5 \times 10^{11}$ ions or electrons per $\mathrm{cm}^{3}$, volume 50 litres, electron temperature $120 \mathrm{keV}$ (some electrons to 3 or $4 \mathrm{Mev}$ ), ions cold, some electrons also cold, beta about $6 \%$, magnetic field about $3 \mathrm{kG}$ midway between mirrors, stable regime easily located.

Since cold plasma continually streams to the ends, the stability can be largely attributed to the conductivity of the cold plasma along lines of magnetic force to the copper cavity ends-a well-known effect sometimes called "line-tying." By adjusting the gas pressure or the magnetic field, 
the plasma can be sent controllably into unstable regimes-by one adjustment into the flute instability and by another adjustment into the mirror instability. Indeed, this may be the first experimental observation of the mirror instability.

Such a plasma has to be modified in three ways toward fusion:

1) The ions have to be heated. We are attacking this in two ways: a) by applying radiofrequency power at a special frequency called a hybrid frequency (no results yet); b) by injecting hot neutral atoms, trapping them by ionization (see description of the INTEREM experiment immediately below);

2) The surrounding vacuum must be reduced well below $10^{-5}$ torr. This is also being worked upon in TVTEREM, with so far a reduction to $10^{-6}$ torr;

3) The magnetic field must be increased. To preserve electron cyclotron resonance this means that the wavelength of the microwaves must be decreased. We have been developing by subcontract with comercial tube manufacturers a) CW oscillators, $1-\mathrm{kW}$ each at $8 \mathrm{~mm}$, and b) CW traveling-wave amplifiers, 1 and $5 \mathrm{~kW}$ at $5.5 \mathrm{~mm}$. We have tried the microwave heating at $8 \mathrm{~m}$ in an apparatus called ELMO. The resonant magnetic field is about $10 \mathrm{kG}$. The $8-\mathrm{mm}$ plasma at $2-\mathrm{kW}$ microwave power is just what we extrapolate from our experiments with $3-\mathrm{cm}$ microwave heating, so we are on solid ground thus far. The experiments with 5.5-m microwaves have not yet been tried, but the resonant magnetic field for them will be $19 \mathrm{kG}$, which is not bad for fusion. 
It should perhaps be remarked that present theory indicates that a population of electrons that are hotter than the ions is undesirable from the point of view of stability and of power balance in the eventual fusion reactor. However, our observation stands: the electron cyclotron plasmas cen be made stable in simple, inexpensive mirror configurations at beta values that are just about high enough for a fusion reactor. We are therefore pursuing our program for a while deliberately in opposition to theory, leaving experiment to tell us whether stability is retained when both the lons and the electrons are not.

\section{INTEREM}

The purpose of the experiment is two-fold: 1) to develop means of reducing the pressure around the electron-cyclotron plasma while still retaining stability; 2) to introduce hot ions by injecting fast neutral deuterium atoms $\left(D^{\circ}\right)$, and trapping them by ionization.

The apparatus (Fig. 4) consists of four magnet coils in line, dividing the vacuum system and the microwave cavity into three regions. The central region serves to contain the hot plasma; it is strongly pumped, microwave power is applied, and the fast $D^{\circ}$ atoms are injected here. The two end regions serve for the generation of cold plasma which penetrates the inner coil throats and stabilizes the central plasma without introducing much gas. Results of the first experiments indicate that stability of the central plasma can be reached at a surrounding pressure of $10^{-6}$ torr, as compared with $10^{-5}$ torr for the apparatus of Fig. 3. We feel that this is capable of further improvement. The first injection experiments ( 40 ma equivalent of $20 \mathrm{keV} D^{\circ}$ ) have been done by turning the $D^{\circ}$ beam on and off while keeping the microwave power on. The decay curve of the trapped fast deuterons 
is found to have a component with a decay time of 0.4 millisecond and a component with a decay time of several milliseconds. The fast deuteron density associated with the short component alone is $3 \times 10^{7}$ per $\mathrm{cm}^{3}$. Experiments are continuing toward fuller understanding.

\section{Turbulent Heating by Beam-Plasma Interaction}

In this work, emphasis is placed upon heating rather than confinement.

The primary difficulty in heating a sample of deuterium from room temperature to $100,000,000$ degrees lies in a physical process called charge exchange. The process causes a loss of ions by interaction of the lons with neutral gas. The situation is particularly difficult because the probability of charge exchange varies with temperature in such a way that up to $100,000,000$ degrees, the more energy you have invested in an Ion, the more likely you are to lose it. Above about $100,000,000$ degrees the probability fortunately reverses; the hotter the ion is, the less likely it is to be lost by charge exchange. Thus there is a charge-exchange barrier that must be overcane in the heating to fusion temperatures.

There are three ways of overcoming the charge-exchange barrier:

I) Heat the ions in a region outside of the plasma confinement region; for example, heat by simple direct-voltage acceleration. This is done in injection-accumulation experiments like the DCX experiments, and several other experiments elsewhere.

2) Heat very quickly. The great theta-pinch experiments, for example, heat their plasma in about a microsecond.

3) Arrange that there be little neutral gas within the volume occupied by the plasma. This has been called the "burned-out" condition; above a certain turning-point the ions are so much 
more numerous than the neutral gas atoms and molecules that they overwhelm the neutrals rather than vice versa.

At Oak Ridge, we have been fortunate in finding a simple arrangement that heats and burms out at the same time. A late version is illustrated in Fig. 5. An electron stream is sent aiong the magnetic lines of force between two mirror coils, and in that region it passes through gas, ionizing as it goes. The beam interacts with the plasma that it thus generates. As early as 1958, two modes of operation had been found, and in the low-pressure regime, which we called "Mode II", it was then recognized that ion heating was taking place. Later, in the more powerful experiment illustrated, we have learned the following:

1) As much as $50 \mathrm{~kW}$ of power can be applied to the electron stream, and the beam-plasma interaction is so strong that of this as much as $7 \mathrm{~kW}$ can be absorbed into about 150 cubic cm of plasma.

2) The core of the plasma is strongly negative (because of the voltage applied to the cathode) so there is a strong radial electric field. This produces on intense rotation in the plasma, about the magnetic axis.

3) The bulk of the ions, at a density of about $5 \times 10^{12}$ per $\mathrm{cm}^{3}$ are heated to about $1 \mathrm{keV}$, as measured in the laboratory system. A few per cent surmount the charge-exchange barrier, and are heated in rosette-like orbits to energies as high as $170 \mathrm{keV}(1,700,000,000$ degreહs). 
4) The interior of the plasma is burned out, as evidenced by the disappearance from the emftted light of the spectral lines of neutral atoms (reduction in intensity by a factor of 1000 when Mode II takes over).

5) When deuterium gas is used, the emlssion of D-D neutrons shows that about a milion fusion reactions take place per second in the central region of the apperatus, between the mirror colls. Many of these, however, may well take place on the walls, and we do not yet know how many fusions take place per second Ithin the pleam proper.

Experinentally, we see several directions in which to improre and exploit this beating method. Work of this general k1nd, under the name turbulent beating, is flourishing in Fussia. To develop this into a fust on reactor, bovever, weans eventually facing the confinement problem, or else making the mod work at mich h1gher pressure. The experiments are tricky, and sensitive to small changes. Here 18 an example of rork that is primarily experimental. Qualitative theory supplies broad guide 11nes, but to try to follow all of the phenomena with detalled, quant1. tative theory would be hopelessly retarding. 


\section{Conclusions and Prospects}

DCX-3 is a laboratory-scale experiment designed to test important aspects of current stability theory. If it shows stability at high density, one might immediately consider an intermediate-scale experiment at higher injection energy, and one might be on the path to a reactor.

DCX-2 is impressive in performance, and must be studied for another year before its future becomes clear.

The electron cyclotron heating mast be pursued into the 19 kllogauss regime to see if the profected density of $5 \times 10^{13}$ electrons per cubic centimeter can be stably achieved.

INTEREM may show us if we can skillfully use cold plasma as a cheap and ready means of stabilization.

The BURNOUT experiments must be pursued toward greater understanding and higher performance. The temperature-density performance is impressive, and the confinement properties need to be further investigated.

Theoretical and experimental plasma physics must be pursued toward mastery of the medium. Engineering, Instrumentation and laboratory technique must develop new and far reaching capabilities. 
Fig. 1 The DCX-I Single-Pass Infection Experiment. A beam of $600-\mathrm{keV} \mathrm{H}_{2}{ }^{+}$ions is injected into a magnetic field between mirror coils; it makes a single loop, and then emerges to be caught and buried on a rotating titaniumcoated target (now shown). While in the trapping region, some of the $\mathrm{H}_{2}^{+}$ions are dissociated, and the resulting $\mathrm{H}^{+}$lons are trapped in the region indicated, which has a volume of about 10 litres. The system has been simple enough to lend itself effectively to a detalled study of the density, space distribution, energy distribution and stability of the trapped proton population.

Fig. 2 Schematic Multiple-Pass Injection Experiment, DCX-2. An $\mathrm{H}_{2}^{+}$beam of about $50 \mathrm{ma}$ of $600 \mathrm{keV}$ is injected at an oblique angle into the trapping region, through a magnetically compensated snout. Usualiy there is a longitudinal vacuum lithium arc through which the spiralling $\mathrm{H}_{2}^{+}$beam passes repeatedly. The 300-kev $\mathrm{H}^{+}$ Ions produced by dissociation in the arc are trapped in the flat-field region between mirror coils; their primary orbits are small enough to miss the snout. The length of the plasma column is about 6 feet.

Fig. 3 Schematic of the Electron-Cyclotron Heating Experiment. A copper cavity about a meter at its largest diameter is placed between two magnetic mirror coils in a vacuum 
tank. Microwave power to about $30 \mathrm{~kW}$ (instead of $5 \mathrm{~kW}$ as shown) is fed into the cavity at a wavelength of $3 \mathrm{~cm}$ (frequency 10,800 megacycles per second). Deuterium gas is also introduced into the cavity, and under the right conditions a stable, high-beta, hot-electron plasma is formed in the central region. The coll marked ICRH is not used.

Fig. 4 The INTEREM Experiment. The hot-1on plasma is in region $A$. Electron cyclotron resonance heating (E.C.R.H.) generates cold plasma in the regions $B$, where gas is fed in. Some of the cold plasma generated in the regions $B$ penetrates the inboard coils and helps to stabilize the hot plasma in region A. Pumps applied to region A hold the pressure there as low as possible.

Fig. 5 The Burnout $V$ experiment on turbulent heating by beam-plasma interaction. An electron beam starts from the f1lament at left, and proceeds toward the right along magnetic fleld Ines through low-pressure gas in the region between mirror coils. It generates plasma in the gas, and then heats the plasma. The very hot ions appear midway between the colls. The plasma volume is about $200 \mathrm{~cm}^{3}$. 


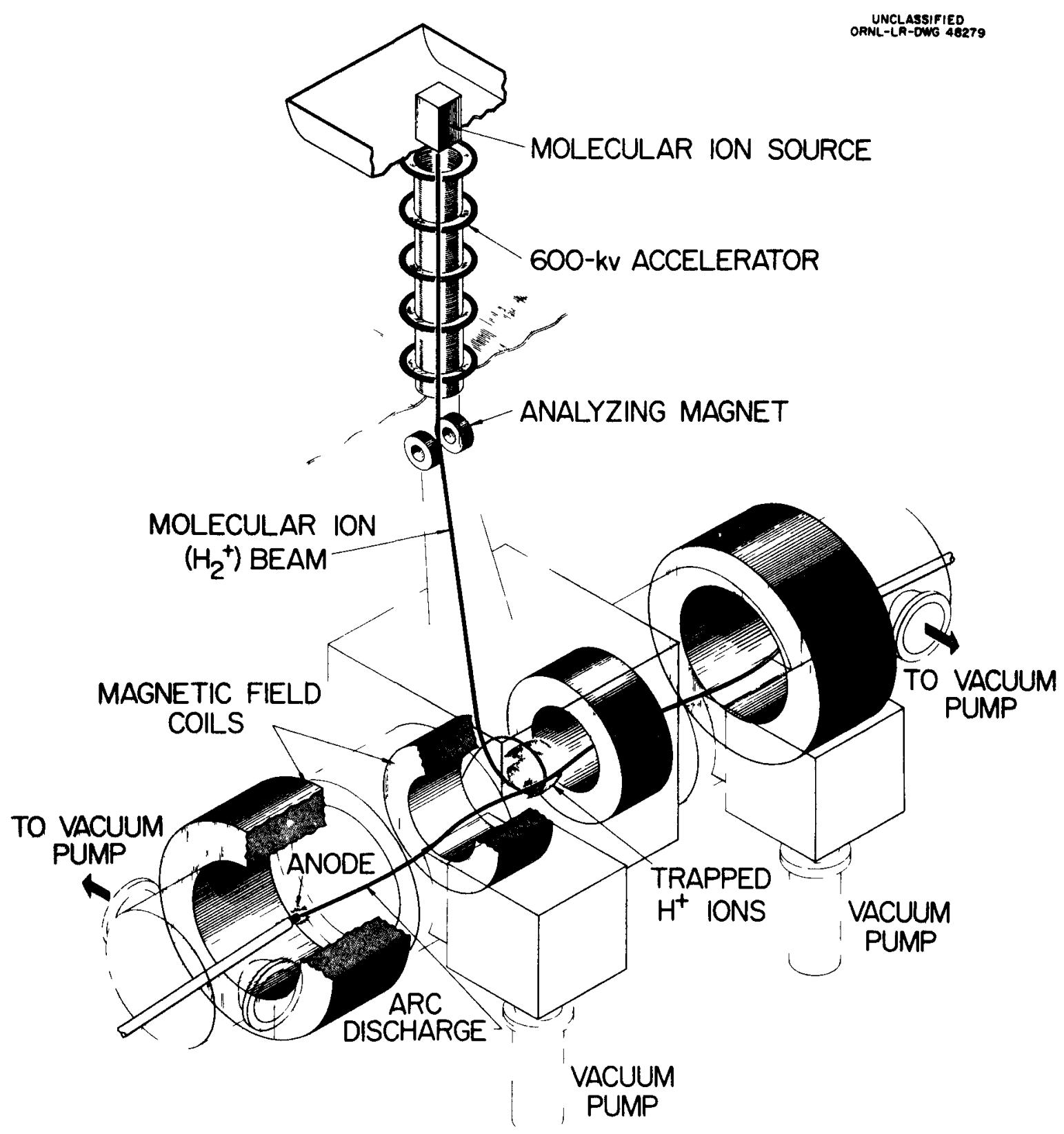

The DCX-1 Experiment.

Fig.? 


$$
\begin{aligned}
& \text { I }
\end{aligned}
$$

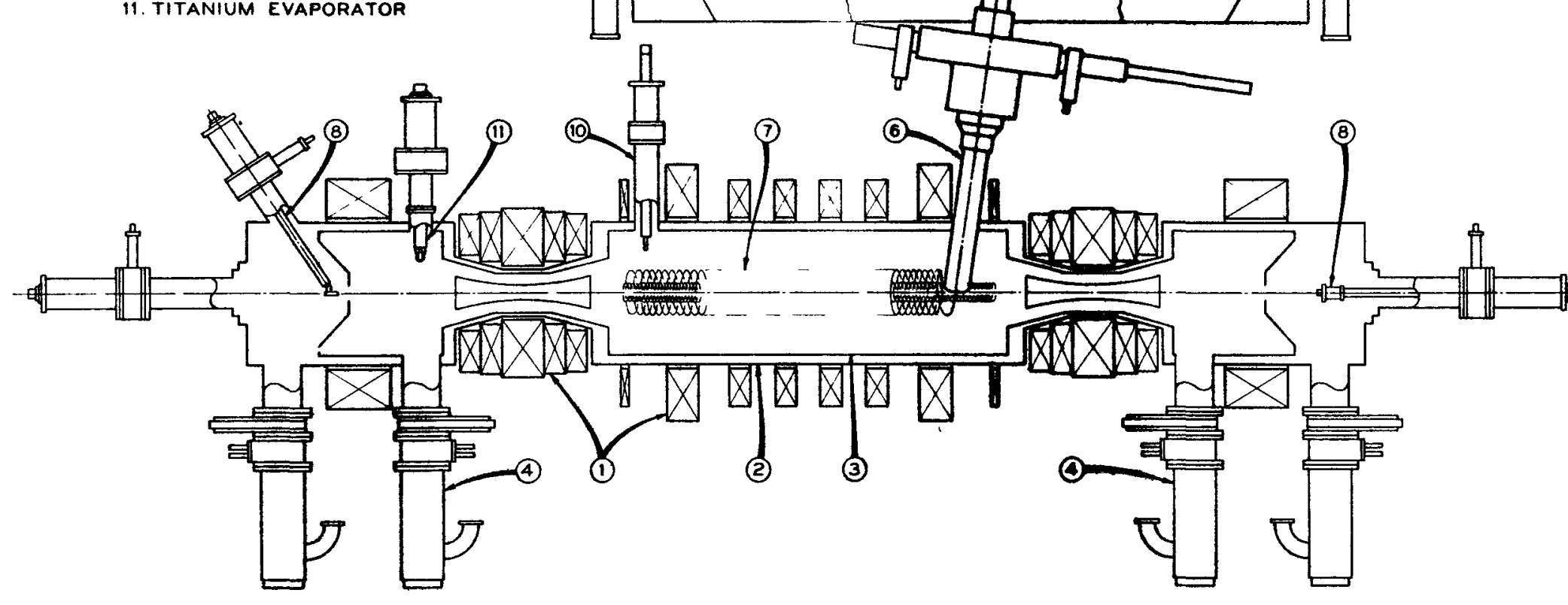




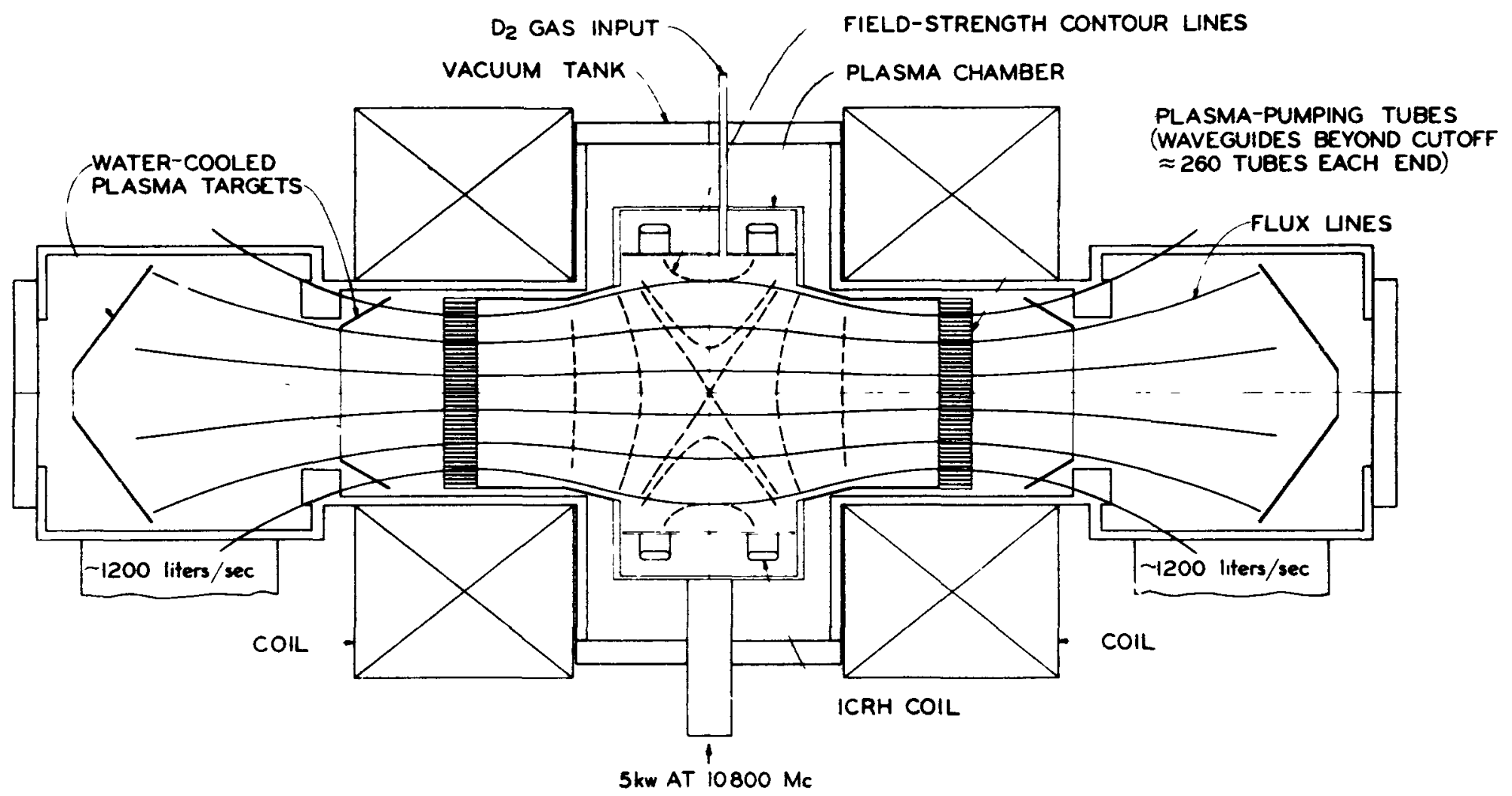

ELECTRON-CYCLOTRON HEATING EXPERIMENT

SCHEMATIC 


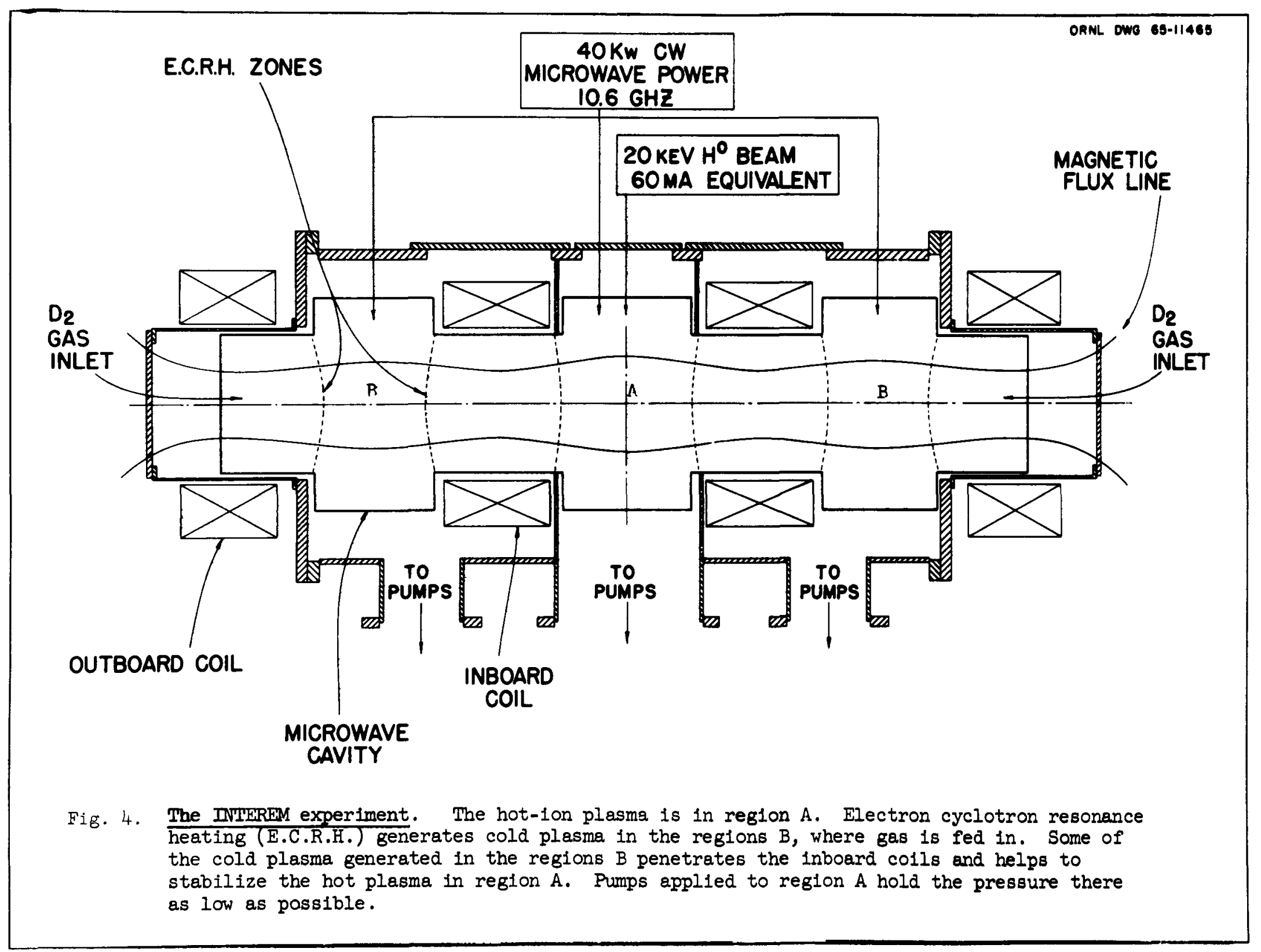




$$
\text { ORNL-DWG 65-3785 }
$$

₹INSULATED

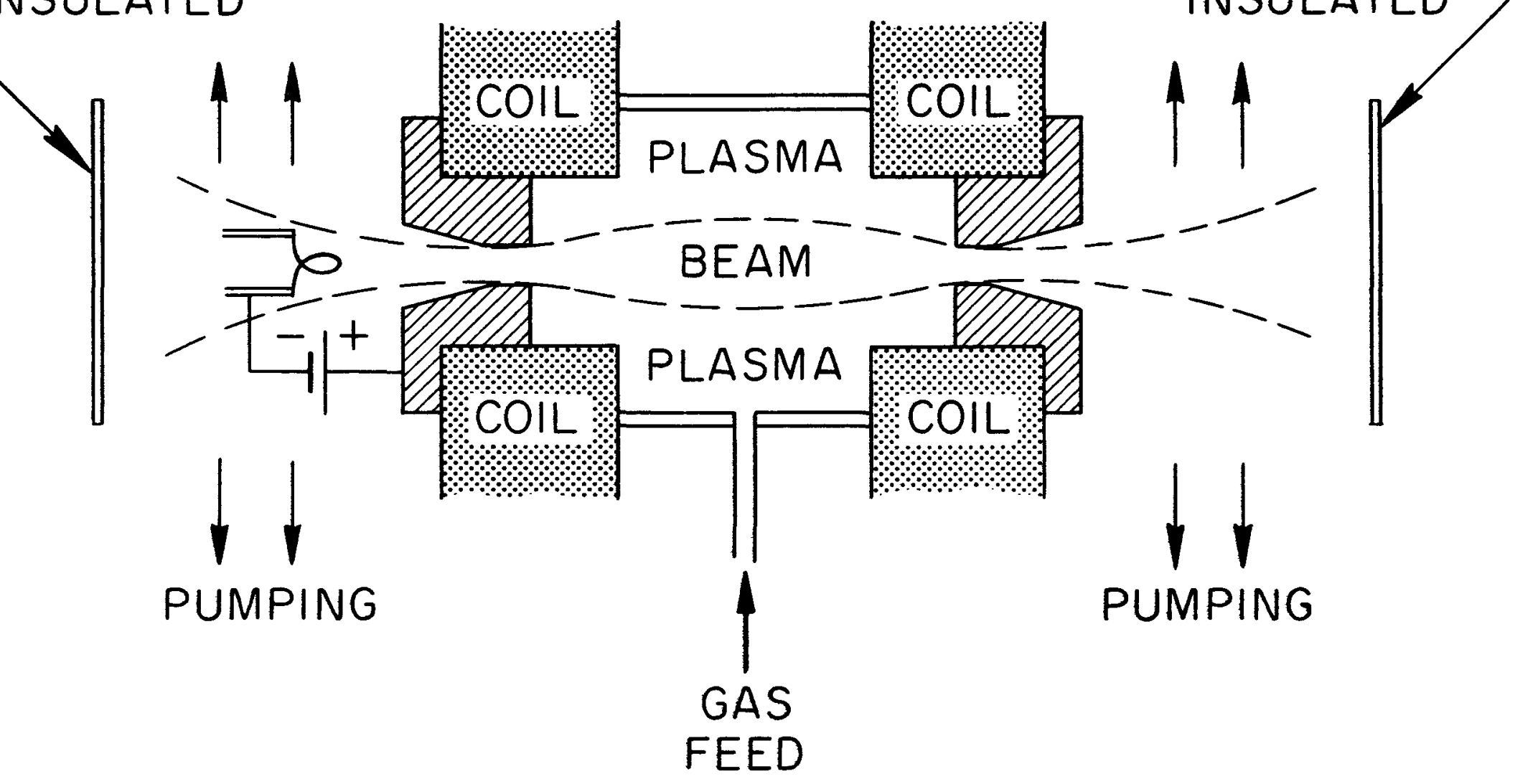

Burnout IV $\sim 18 \mathrm{~cm}^{3}$

Burnout $\mathrm{V} \sim 320 \mathrm{~cm}^{3}$ 
3. Los Alamos Scientific Laboratory

\section{INTRODUCTION}

The principal obstacle to the achievement of controlled thermonuclear power is instability, which causes loss of hot plasma from the confining magnetic bottle and cuts down the time of confinement. It is a physical law-that to make a power producing thermonuclear reactor, the plasma, assumed hot enough (a condition we now approach), must also achieve a high enough value of the quantity (plasma density $x$ confinement time) in order to pay back the energy cost of confining and heating the plasma. According to this rule, one can trade off confinement time against plasma density. At Los Alamos we have long worked with methods of heating and confining plasmas which involve pulsed magnetic fields at much higher plasma densities than those found (or even envisaged) in devices where the plasma is built up in static magnetic bottles. Part of our program, in common with most of the U. S. effort, is to attempt to stabilize the plasmas produced by our techniques and to see what physical phenomena actually limit stability. In the shorter confinement times afforded by our higher densities, there may be fewer instabilities to be overcome. No one can yet predict whether a fully stabilized, static magnetic confining bottle will ever be achieved. It is vital therefore to devote some part of the U. S. effort to approaches which could succeed with only partially stabilized, or even completely unstabilized magnetic confinement. Such experiments at extreme densities represent the rest of our program. This is the essential philosophy behind the Los Alamos pulsed, high density plasma experiments.

Following these principles, we have a notable success in the form of a strong (100 wat $t$ ) thermonuclear reaction in a dense plasma, confined for about $5 \mu \mathrm{sec}$ in the Scylla IV experiment.

Careful consideration of reactors based on the above principles and the success we now have indicates essentially three promising regions for attack:

1. To extend the duration of confinement of the Scylla IV plasma at the maximum pressure that the mechanical strength of external magnetic coils will allow. Our present Scylla IV device is open-ended and the duration is limited by the plasma running out at the ends. To get to longer times we must close the ends into a torus (Scylla closed $=$ Scyllac).

This involves considerable increase of size, pulse energy, and much complication of principle. Scyllac involves $\$ 5$ million to build and $\$ 1$ million to house. To achieve its goal of approaching within 1/100 of a power producing reactor, the confinement time will have to stretch out as long as $100 \mu$ secs. It is expected that some recourse to dynamic stabilization ( 3 below) may be needed if static methods do not give sufficiently long confinement.

2. Other experiments are being explared, all of them aimed at much higher densities and still shorter times. Among them is the dense plasma focus experiment which is an outgrowth of plasma gun research. Other experiments involve the compression by a pinch of a plasma already heated by a Scylla machine (Columba experiment) or by a hydromagnetic gun. These experiments are a step 
still further away from the risk of being struck down by instabilities, but at the cost of a still more impulsive release of power. There are two points that should be made here: (a) Support for the high density pulsed approach to thermonuclear power can be drawn from the incontrovertible fact that, instabilities notwithstanding, the only net power producing thermonuclear reaction known on earth is a high density pulse device - the hydrogen bomb. Clearly, somewhere between the ideal of a steadily burning device, which may be impossible, and the hydrogen bomb power source, wh: $=\mathrm{h}$ is impracticable (or at least inconvenient) should be some much milder s: ctical pulsed device; (b) It should be noted that this impulsive approach does not introduce any hazard from loss of control any more than does the internal combusion engine which it resembles. There is too little fuel in a thermonuclear reactor to be dangerous.

3. Further, in the event of failure to achieve static stable magnetic confinement, one can consider magnetic bottles in which the ingtabilities grow out toward the walls, but are reconstructed and smoothed back to zero again periodically, by the superposition of relatively small rapidly changing magnetic fields. This is known as dynamic stabilization. It may be obligatory in Scyllac, and would enormously simplify the achievement of a reactor by the highly pulsed approach (2). It may even permit of a DD reactor - which all would prefer to DT if it can be done.

The present Los Alamos situation in round numbers is 50 people, annual budget $\$ 2 M$.

What we need to accomplish the above program in a prudent way will require minima1ly:
1. Approximate doubling of the staff (to 100 people) spread over five years.
2. Annual budget expansion to $\$ 4 M$ spread over the same period.
3. Expedition by all means possible of the \$1M Scyllac bullding line item (potentlally a bottleneck).
4. Provision of $\$ 5 M$ Scyllac construction item.

\section{SCYLIAC}

\section{A. Scylla Theta Pinches}

The Scylla theta-pinch program, initiated in 1957, produced the first laboratory thermonuclear plasma. Over the past 9 years, Scylla has evolved into the present Scylla IV experiment, and extensive measurements have been made of the plasma properties. There have been four Scylla experiments. The basic measurements performed on these devices are summarized in Fig, 1 . (The Scylla plasma has approximately the same temperature and radiating properties as the sun's outer atmosphere (corona). Spectroscopic data from this laboratory plasma have been compared directly with rocket measurements made of the sun.) The principles of operation of the theta pinch are given in Appendix $A$.

For some years, the sum total of the evidence has been that scylla theta-pinch plasmas have the following characteristic values: density, 


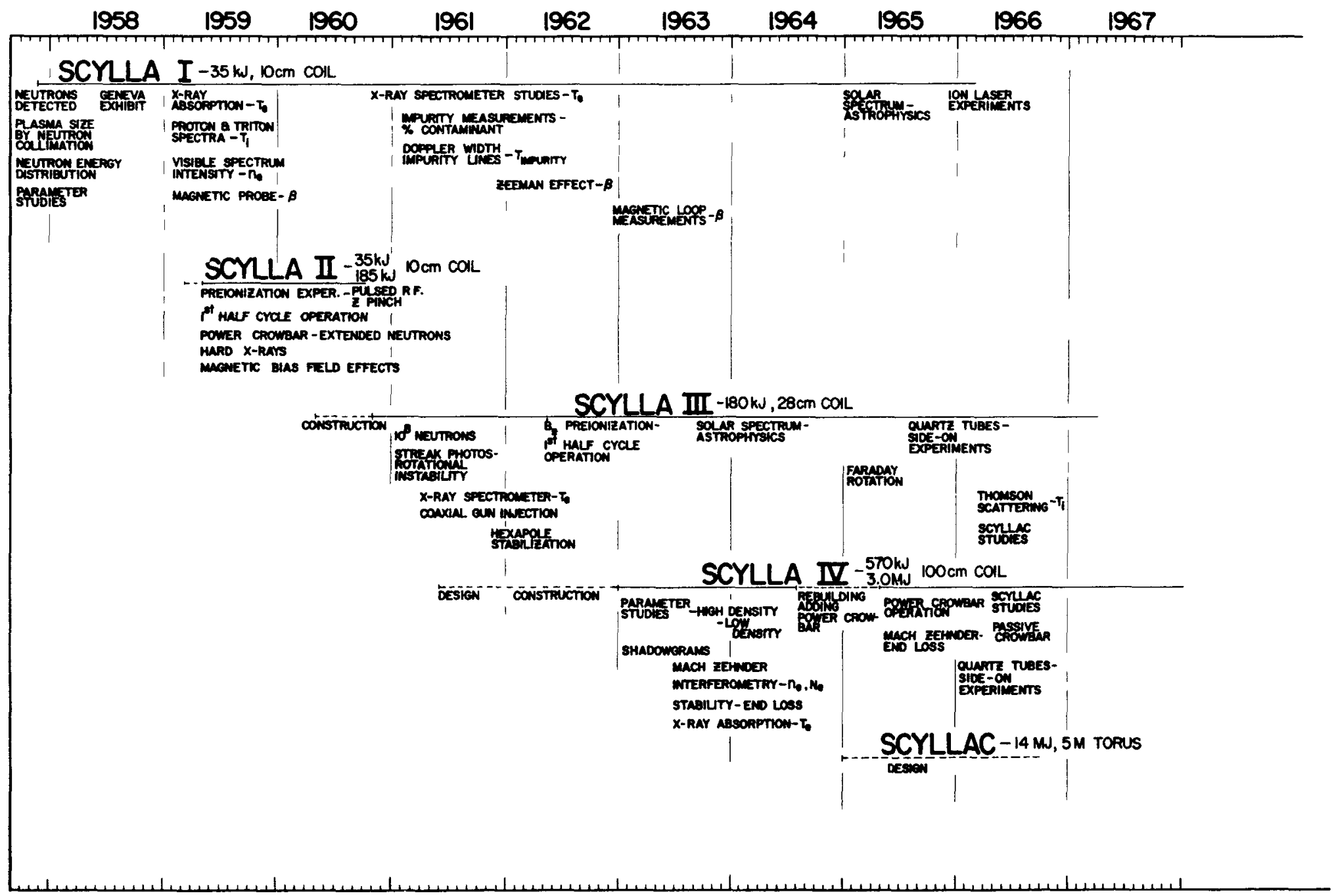

Fig. 1. Summary of Experimental Work on the Los Alamos Theta-Pinches. 
$6 \times 10^{16}$ ion $/ \mathrm{cm}^{3}$; electron temperature, $300-1200 \mathrm{eV}\left(3,300,000\right.$ to $\left.14,000,000^{\circ} \mathrm{K}\right)$; ion temperatures 1.3 to $2.6 \mathrm{keV}$; and $\beta$ close to unity. Neutron yields range from $5 \times 10^{6}$ per pulse (Scylla I) to $2 \times 10^{9}$ per pulse (Scylla IV) with mean

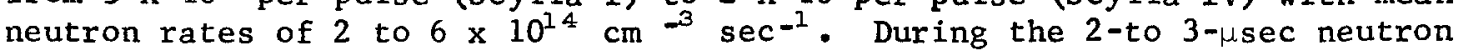
emitting lifetime of the firebal1, an ion characteristically makes several hundred reflections from the magnetic wall in the radial direction and an average of one to two ion-ion collisions. It can thus be classed as a confined, strongly thermonuclear reacting plasma, but is not necessarily Maxwellian. These results are obtained with deuterium filling pressure about 0.1 torr (high-pressure regime), and negative bias magnetic field is required.

More recently Scylla IV has been operated in an entirely new low-pressure regime $\left(\mathrm{P}_{\mathrm{D}_{2}}\right.$ : 10 to 50 mtorr $)$ without bias magnetic field. Interferograms taken in this low-pressure regime show a plasma density lower than that for the highpressure operation and reduced plasma volume. Pressure balance (at $\beta=\frac{1}{2}$ ) and neutron yield measurements place the mean ion energy in the 1.5- to 5-keV region. The low-pressure operation corresponds to the original conception of Scylla behavior. The plasma parameters, in particular the ion temperature are predictable from classical shock and adiabatic compression considerations. Characteristic values are: density $2 \times 10^{16}$ ion $/ \mathrm{cm}^{3}$; electron temperature, $300 \mathrm{eV}$; mean ion energies 1.5 to $5 \mathrm{keV}$. There is evidence that $\beta$ is lower $(\sim 1 / 2)$ than in the high-pressure regime. In addition, impurity levels are only about $1 / 50$ those of the old, high-density regime. Interferometric techniques, show a rapid loss of plasma out the ends of the compression coil. This end loss, occurring in about $5 \mu \mathrm{sec}$, is the present limiting factor on containment.

The Scylla apparatus at Los Alamos has been a striking success in getting plasma hot enough and dense enough. However, it is down by a factor of 500 to 1,000 from the product $n T$ (density $x$ time) necessary to repay the energy spent on it; i.e., to show an energy profit.

Magnetic confinement bottles fall into two categories, open and closed either of which may be pulsed or steady. Scylla IV is an open pulsed system and is, therefore, subject to end loss. The proposed scyllac system is a closed pulsed system which eliminates end loss, and is designed to carry the Sherwood program at Los Alamos to larger (density $x$ time) than Scylla IV is able to reach. The purpose of this experiment will be to study the toroidal stabilization problem and to achieve long confinement times. The elimination of end losses will allow concentration on the study of plasma stability and purity. Confinement times of about $100 \mu \mathrm{sec}$ will be possible with the particular design proposed.

\section{B. The Scylla Toroidal Theta-Pinch}

1. Methods of Stabilizing

When a theta-pinch plasma contained by a longitudinal magnetic field is bent into toroidal form, the equilibrium, as well as the neutral "interchange" stability which occurred when the magnetic lines were straight, are 1ost. If the lines were simply bent to a mean radius of curvature $R$ on the plasma axis, then the plasma would move outward at a constant acceleration. 
Assuming a plasma like that in Scylla IV, it would "drift" to the wall in about

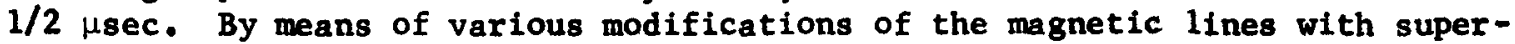
imposed transverse fields, their curvature can be made to vary (usually periodically) In sign, providing either cusped or "bumpy" toroidal gecmetries. However, if these magnetic lines in contact with the plasma are not to intersect the wall of the toroidal discharge tube (the bumpy case) it is geometrically necessary that there be regions of "bad" curvature with respect to the growth of interchanges. In such regimes the lines curve away from the plasma, and this is known to cause interchange instabilities, or rippling of the plasma surface parallel to the magnetic lines.

In Scyllac we shall apply three basic methods to stabilize the drift and the higher-order interchange modes, using three possible methods of stabilization or combinations thereof. Only research can determine which will finally be successful. (a) The first applies theoretically at rather low $\beta$ and consists of providing periodic magnetic field configurations by means of auxiliary multipole conductors such that the average value of magnetic field has a minimum near the axis of the torus ( $\int \mathrm{d} l / \mathrm{B}$ stabilization). It must be determined by experiment whether this method of stabilization extends to the high $\beta$ values (roughly 1/2) present in Scylla IV-type plasmas. (b) The second "static" method achieves theoretical equilibrium at the $\beta=11$ imit. It consists of providing vacuum magnetic fields in which a completely diamagnetic plasma will experience zero net force and hence an equilibrium. The plasma surface may be a cusped one or a bumpy one. The cusped equilibrium is stable, but suffers from cusp loss. In the bumpy case there is some mathematical expectation that a given set of stabilizing conductors may provide equilibrium at both low $\beta$ and $\beta=1$. In the $\beta=1$ limit, these bumpy configurations are theoretically unstable to interchanges, and a third type (c) of stabilization - dynamic stabilization may finally be necessary. This consists of superimposing on the longitudinal field $B_{0}$ of the $\theta$-pinch a relatively small azimuthal field (from a longitudinal current), alternating rapidly compared to the growth time of the interchange modes that it must stabilize. It may be that our plasmas will lie sufficiently close to the theoretical low- $\beta$ regime that dynamical stabilization is not necessary. If the equilibrium can be assumed by $\beta=1$ bumpiness, then the dynamical system will be relieved of having to deal with the $m=1$ interchange, which is the fastest. In this case theory indicates that the higher-m modes will be sufficiently slow that dynamic stabilization is much easier.

\section{Engineering Features}

Our basic experimental method is to retain the known dynamical heating of present linear theta-pinches in a torus of large aspect ratio. It is known that transverse stabilization fields, present at the time of the initial implosion of the theta-pinch spoil the plasma heating. Therefore we intend to apply the main compression field, followed by the stabilization fields after a sufficient time lapse that they do not interfere with the intial heating of the theta-pinch. At the larga aspect ratio (slight curvature or large overall diameter) of Scyllac, the drift time $(\sim 1 / 2 \mu \mathrm{sec})$ of the plasma to the wall will be sufficient to allow this separation of the heating and stabilization functions. 


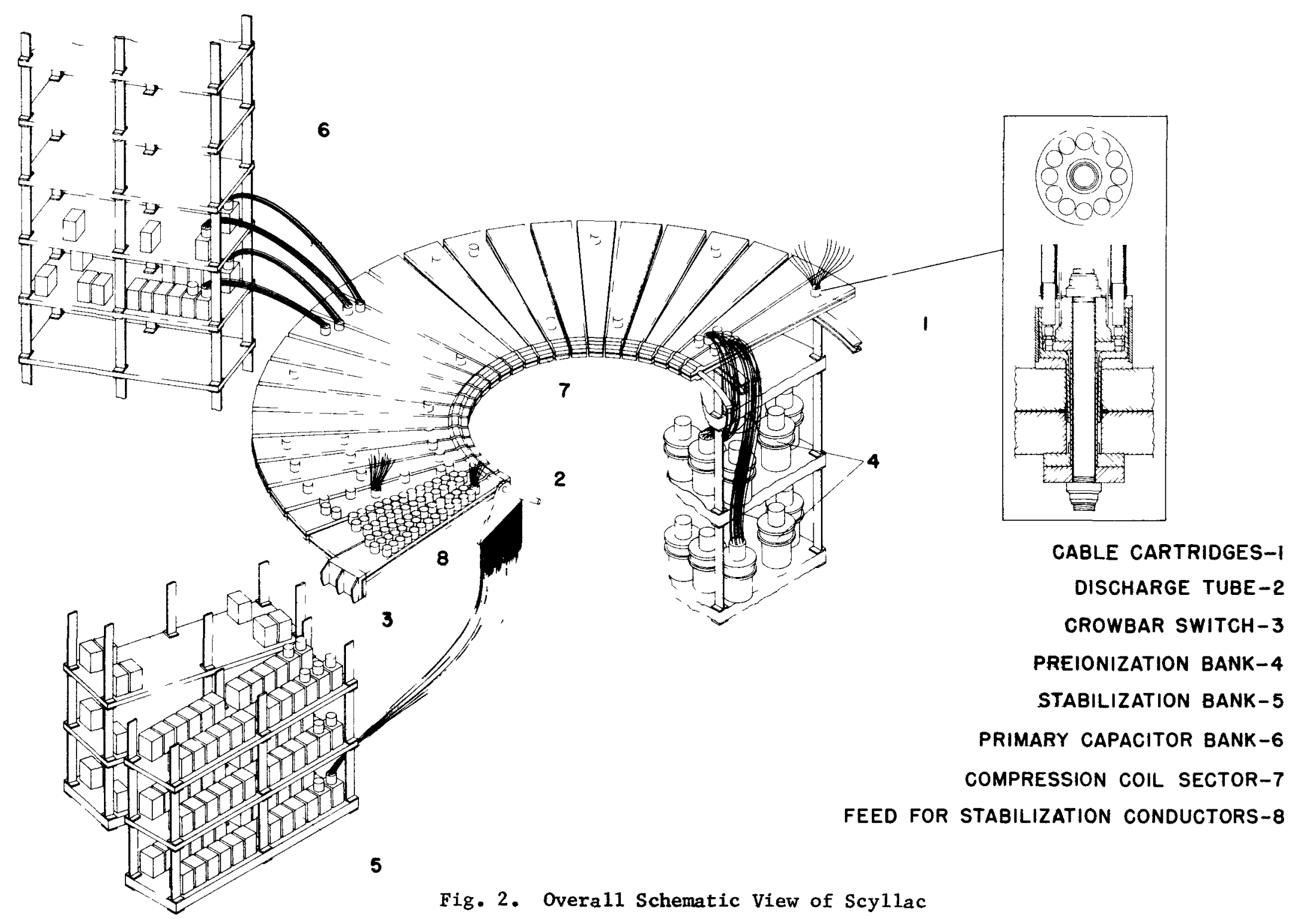


The basic engineering problems of Scyllac lie within the experience developed over 9 years of engineering of the theta-pinch systems at Los Alamos. However, owing to the magnitude of Scyllac, new problems arise in quality control of components and systems engineering, as well as detailed design of the larger device. A qualitative view of some of the main components of Scyllac is shown in Fig. 2. It consists of a toroidal compression coil 5 meters in diameter and cut into 48 angular sectors. At the 48 joints are the feeds of the stabilization windings (only one of which is shown). Capacitors occupy racks both above and below the thick aluninum-alloy collector-plate and coil segments. Many coaxial cables connect the two. Roughly speaking, the capacitors above floor level drive the theta-pinch heating, while those below (about 40 percent of the total) drive the stabilization conductors. The larger capacitors near the center below are for preionizing the deuterium plasma. The crowbar switches shown at the rear of the segments are mechanical switches driven by electrically exploded foils. Their object is to shortcircuit the compression coil after it has been energized by the capacitors and to "lock" the compressing magnetic field in it for the $100 \mu \mathrm{sec}$. Important new engineering problems to be solved and tested consist of the development: of these switches, of the stabilizing circuits, and of a new method of connecting the thousands of coaxial cables from the capacitors to the collector plate (see the inset in Fig. 2). Engineering is proceeding and these techniques are being tried on Scylle IV and on specially constructed models.

\section{Appendix to Section II.}

\section{Principles of the Theta Pinch}

The essential processes by which the thermonuclear plasma in a thetapinch is produced and contained are illustrated in Fig. 3. A massive, singleturn coil is rapidly filled with magnetic field as a capacitor bank is suddenly switched on. The inrushing magnetic field surrounds deuterium plasma inside the coil but cannot penetrate it because of the plasma's high electrical conductivity. Therefore a large magnetic pressure is built up between the outside of the plasma and the coil wall. This pressure drives the outer surface of the plasma rapidly inward [Fig. 3(a)]. During this early dynamic phase, a shock wave runs into the plasma to convert inward plasma motion to heat, producing temperatures of a few hundred electron volts. This dynamical process occurs before the capacitor has lost a great deal of its energy, i.e., long before the energy of the magnetic field in the coil has built up its final value. As the magnetic field increases, it contains the heated plasma from the dynamic phase and slowly (adiabatically) compresses it during the quiescent phase of the discharge $[\mathrm{Fig} .3(\mathrm{~b})]$ further heating to a few thousand electron volts. Finally when all the electrostatic energy has left the capacitor bank and been converted to magnetic energy in the coil, there is a long, ellipsoidal plasma held by the magnetic field away from the wall.

The 100,000-gauss magnetic field of the fast, primary capacitor bank of Scylla IV is representative of those found in $\theta$-pinches. The magnetic field is furnished by $8,000,000 \mathrm{~A}$ of current spread over the inner surface of the coil and flowing in a circle around it. The initial voltage to wich the Scylla IV capacitor bank is charged is $50,000 \mathrm{~V}$, and the electrostatic and 


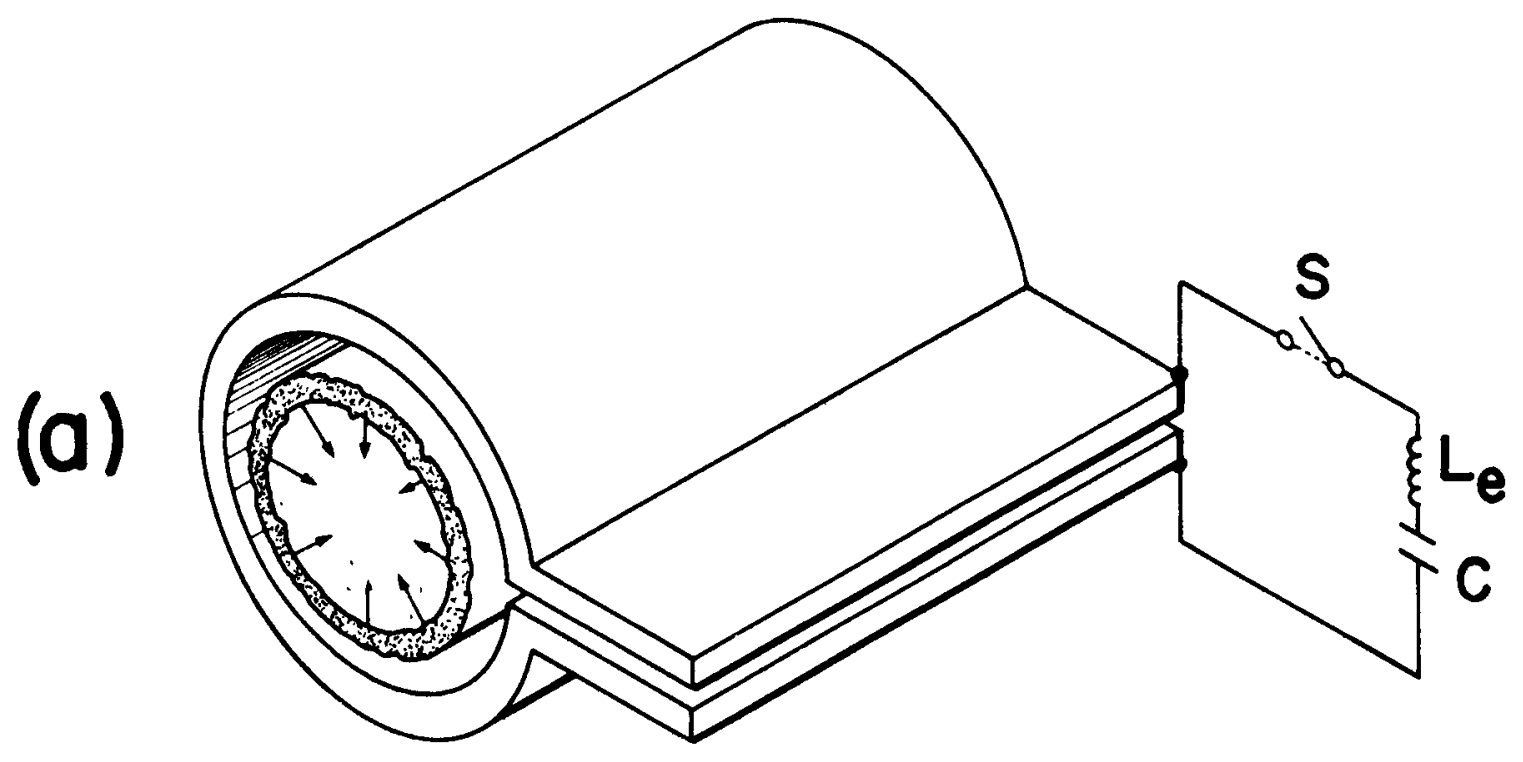

DYNAMIC PHASE

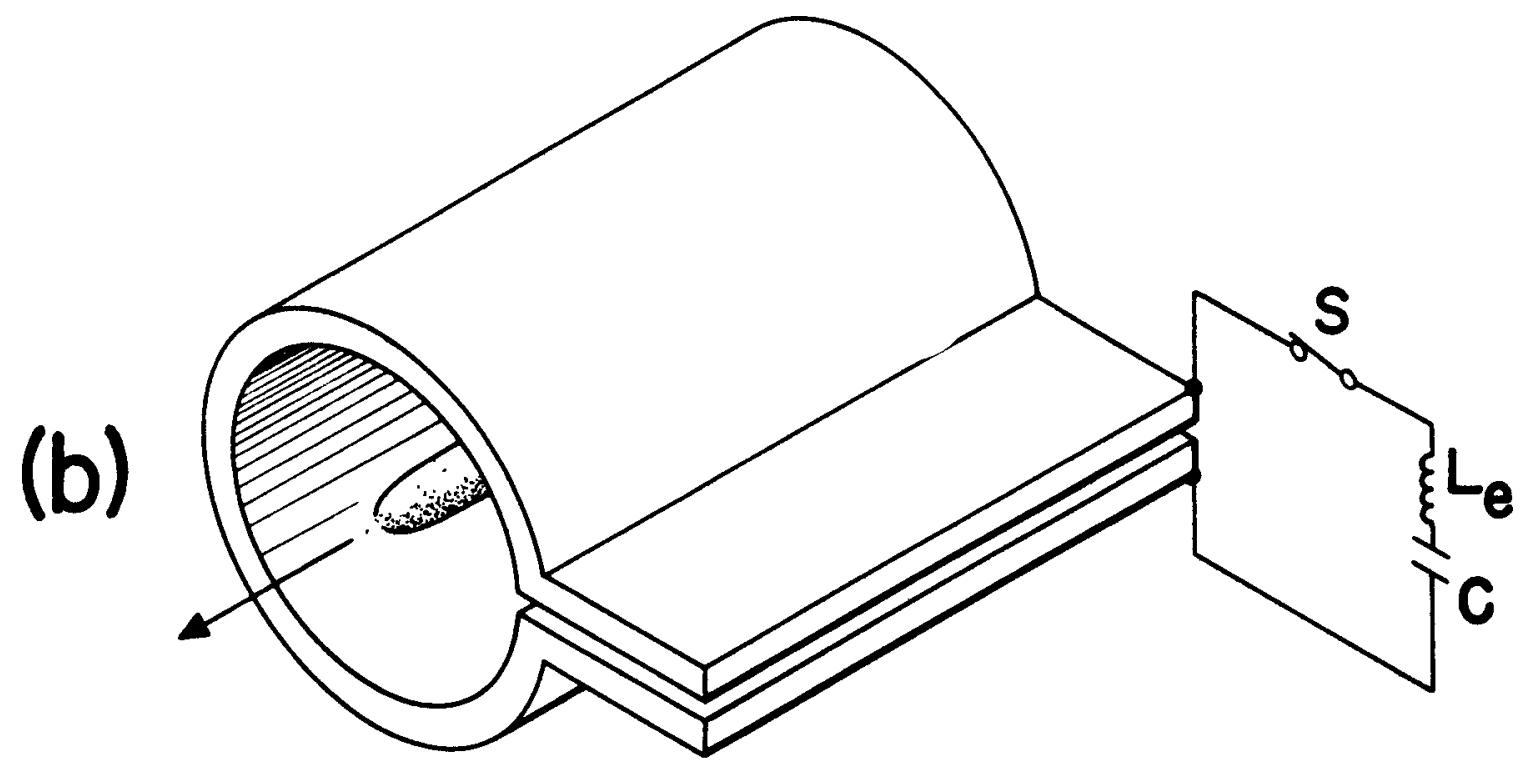

QUIESCENT PHASE

Fig. 3. Simplified Schematic View of a Theta-Pinch. 
magnetic energy is about $600,000 \mathrm{~J}$.

The Scy1la experiments at Los Alamos differ from those carried out in many other laboratories in that the capacitor voltage is large and that a large fraction of the voltage is transferred to the compression coil through the low inductance $L_{c}$ of the external circuit. It is this high voltage (per unit circumference of the coil inner surface) that determines the speed of the initial dynamic phase and the heating by the shock wave. Fig. 1, while correctly illustrating principles, is greatly over-simplified. The plasma is contained inside an evacuated cylindrical discharge tube made of refractory alumina ceramic or fused quartz which is fitted closely inside the compression coil. The tube is evacuated to $5 \times 10^{-7}$ torr ( 1 torr is approximately one thousandth atmospheric pressure) before the admission of deuterium gas to a pressure of 0.01 to 0.1 torr for producing the plasma. The switch $\mathrm{S}$ which allows current to flow from the capacitor bank actually consists of many parallel spark gaps, each feeding many parallel cables which carry the millions of amperes that flow in the coil. The current collector which feeds the coil slot is a pair of thick $(7.5 \mathrm{~cm})$ aluminum alloy plates, bolted and clamped together to withstand the large magnetic forces from the currents flowing between them.

\section{COLUMBA - A HOT DYNAMTC Z-PINCH}

For the release of energy in a thermonuclear reactor to occur in times like $10 \mu \mathrm{sec}$ it is necessary that the particle density in a DT mixture be greater than $\sim 10^{19}$ per cc. At the temperatures required in the reactor, $\sim 10 \mathrm{keV}$, the plasma pressure is high, $\approx 1.6 \times 10^{5}$ atmospheres or $\sim 1,200$ tons per in $^{2}$. To contain this pressure by magnetic fields requires $\sim 2$ megagauss. As all metals have yield strengths about a factor ten smaller than that required to support these pressures it is clear that these confining magnetic fields can not be produced by currents passing through material coils. However, these large magnetic fields can be generated by passing realizable currents through the plasma itself. For example, a current of 10 million amperes flowing through a plasma column of $1 \mathrm{~cm}$ radius produces a magnetic field of 2 megagauss on its surface and an inward pressure of $\sim 1,200$ tons per $i^{2}$. These considerations, of course, are the basis for the early $z$-pinch experiments at LASL with the Columbus and Perhapsatron experiments and elsewhere.

A11 previous attempts with fast $z$-pinches were severely limited by the fast growing instabilities which prevented an increase in plasma conductivity and caused a violent wriggling of the plasma column about the discharge tube and finally striking the tube wa11. The maximum temperatures that could be achieved were low, $\sim 20 \mathrm{eV}$, and possible stabilizing factors such as high conductivity and finite Larmor radius could not become important.

Today, however, hot dense plasmas have become available in the laboratory and there is the possibility of circumventing the initial difficulties found with the $z$-pinch and producing a hot, high density $z$-pinch. The Columba experiment, therefore, is an attempt to superimpose on a hot plasma, in particular a Scylla plasma, a fast z-pinch. The Scylla plasma has been extensively studied and plasmas of density $\sim 3 \times 10^{16}$ per $c c, T_{i} \approx 2 \mathrm{keV}$ and 
$\mathrm{T} \approx 500 \mathrm{eV}$ can be reliably produced. When the $z$-pinch is initiated on this hot dênse plasma the large self-magnetic field will further compress and heat the plasma and it is expected that a new regime of $z$-pinches can be investigated.

The first step in the program is the superposition of the $z$-pinch on the Scylla plasma without trapping a significant amount of magnetic flux from the $\theta$-pinch. A little of this flux will help to stabilize against the $m=0$ instability but too much will make a significant compression by the self-magnetic field impossible. Once this has been achieved the containment and stability can be investigated. In the long run it will probably turn out, as it has with other approaches in the CTR program, that the confinement of the hot $z$-pinch will not be sufficiently long. In this case we hope that the stability will have been increased to that point where further stabilization by time varying magnetic fields with frequencies low enough to be practical can be investigated.

As for the hardware, it is expected that the technology developed at IASL in the design and operation of both the fast $\theta$ - and $z$-pinches will be combined in these experiments. As the emphasis is on fast systems there is much that has still to be done to extend our knowledge and techniques in developing hardware to handle multi-megamp discharge currents at many tens of kilovolts. Also we are just beginning to become familiar with short bursts of R.F. power in the hundreds of megawatt range which will have to be increased if significant work is to be done on dynamic stabilization.

\section{DENSE PLASMA FOCUS EXPERIMENT}

The production of a hot dense plasma focus beyond the end of the hydromagnetic gun center electrode, Fig. 4 is believed to be by a fast $z$-pinch mechanism in which the current sheath is driven radially inward or focused on axis beyond the center electrode by the pressure of its self-magnetic field.

Experiments with the plasma focus using pure deuterium gas at pressures of 2 - 3 torr and a $90 \mu \mathrm{F}-20 \mathrm{kV}$ condenser bank show the following plasma properties: 1) a plasma density $\left.\mathrm{n} \sim 2-3 \times 10^{19} / \mathrm{cc}, 2\right)$ a maximum electron temperature $\left.T_{1} \sim 5 \mathrm{keV}, 3\right)$ a volume of $\left.1-5 \mathrm{~mm}^{3}, 4\right)$ a neutron production of $\sim 10^{10}$ neutrons in a time of $0.15 \mu \mathrm{sec}, 5)$ a spatial neutron anisotropy $<5 \%$, and 6) a total energy release in soft $x$-rays $\left(T_{e} \sim 5 \mathrm{keV}\right)$ of $\sim 50$ joules.

A comparison of neutron production between pure deuterium and a 50/50 gas mixture of deuterium-tritium $(D-T)$ has been made at reduced voltages. For deuterium, the neutron yield was $\sim 4 \times 10^{9}$ in a time of $0.15 \mu \mathrm{sec}$; for $D-T$, the yield was increased $\sim 100$ fold to $3.6 \times 10^{11}$ in a time of $0.22 \mu \mathrm{sec}$. The increase in the lifetime of the plasma focus may be significant and suggests a stabilization mechanism perhaps due to the heavier ion mass $(T)$, i.e., finite Larmor radius effects.

Experiments are planned to investigate in more detail 1) the particle density as a function of energy and pressure using the interferometric technique, 2) the dependence of the electron temperature on pressure and energy by the time-resolved dual channel $x$-ray spectrometer method, 3) the loss of energetic ions or neutrals along the axis of the pinch -- this measurement may help to 


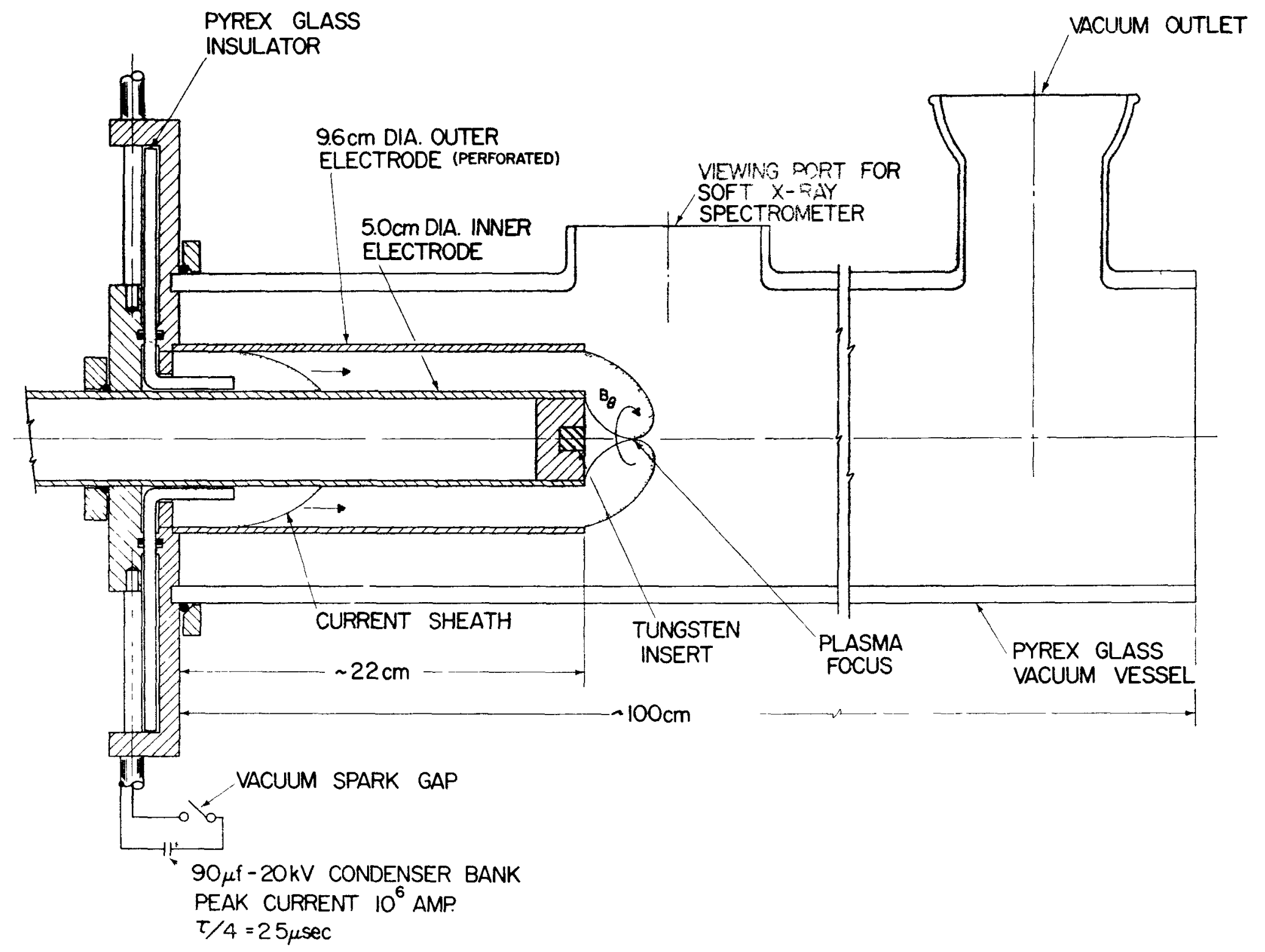

Fig. 4. Schematic of the coaxial plasma gun showing the formation of the dense plasma focus by the collapsing current sheath. 
answer the question whether the plasma focus is sustained by the influx of energetic particles into the plasma to offset the losses expected along the axis or is contained by the pressure of the magnetic field, 4) the scaling of the plasma focus device as a function of the condenser energy and the electrode geometry, 5) the possibility of coupling several plasma focus systems in a multiple array -- it appears possible to position the plasma focus by properly curving the coaxial geometry, 6) the spectral distribution and intensity of the soft $x$-rays from the plasma focus, and 7) the magnetic field and current distribution in the vicinity of the plasma focus.

\section{HYDROMAGNETIC GUNS, WITH ASSOCIATED AND DERIVED RESEARCH}

Hydromagnetic guns have been studied in Los Alamos over a period of 8 years. The present coaxial gun is the result of empirical search and optimization of gun parameters, while keeping the stored energy involved small and approximately constant. In its present form it provides a relatively simple and efficient method of producing experimentally useful quantities of deuterium plasma at temperatures of interest for thermonuclear devices. About 1 kilojoule of kinetic energy of useful fast plasma is produced from an initial stored energy of 6 kilojoules. Whether the gun can be made useful in the charging of thermonuclear reactors depends primarily on whether the amount of energetic plasma produced can be scaled up by a large factor, say 1000 , without undesirable side effects such as excessive impurities. A serious program for the scaling up of guns is in preparation and will be continued over the next few years, assuming the initial results to be favorable. The first scale-up step is by roughly a factor 10 in energy. This will be attempted at $50 \mathrm{kV}$ instead of the present 20 . It requires a large vacuum chamber which is presently in procurement.

The mechanism of coaxial hydromagnetic gun operation is imperfectly understood even now after many years. The new installation with its large vacuum chamber and improved experimental conditions will provide the opportunity to extend recent studies of the mechanism of acceleration of the fast plasma component. Needless to say, an improved understanding of the mechanisms involved will be useful if not essential to scaling the gun to higher energies.

Associated with gun development has been a program of injection experiments in which the interactions of gun plasma with guide and containment fields have been studied. Injection, both parallel and transverse to the magnetic field, has been investigated. At present a series of studies of transverse injection is quite active and presumably it will remain so for some time. Transverse injection, through complicated electric field depolarization phenomena, appears to offer a practical method of charging closed field line containment devices with gun plasma.

Hydromagnetic guns are complicated in their operation and frequently behave in unexpected and spectacular ways. An example of the development of one such behavior mode is the dense plasma focus, which started as an attempt to use a coaxial gun to inject a fast linear pinch. In the following up of the plasma focus phenomenon the injected pinch experiment was 
dropped. It is planned to take it up again in the immediate future with a somewhat different approach from the one leading to the plasma focus. This approach has the advantage of somewhat more knowledge of the gun plasma than was available for the previous experiment.

The hope in this peripheral type of explatory research is to run onto new phenomena which might make possible the development of high density, fast pulsed machines with reactor possibilities. The fact is that most of the present fusion research machines involving the hydromagnetic behavior of plasmas are the result of the empirical following of experimental phenomena. Every new experiment in this field leads to a surprise. If the surprise is a pleasant one, advantage can be taken of it, and if work is continued long enough, a reasonably complete understanding can be achieved.

\section{THEORY}

The mathematical physics effort is divided into three main parts, two of which are closely tied to the experimental programs and to planning for a toroidal theta pinch (the Scyllac proposal). The first line of investigation concerns the existence, nature of, and stability (hydromagnetic, kinematic, and universal) of straight and toroidal plasma columns, both in the low and high beta limits. The methods of attack are both numerical and analytical, with attention given to reasonable overlap of the approaches for flexibility. The second program (a sophisticated computational one) carries out pulsed magnetic field calculations in systems with generalized cylindrical symmetry, the results having relevance to the design and analysis of experiments in cusped and multipole confinement devices, in the low beta limit. The third effort is directed toward fundamental plasma theory such as kinetic theory, coherent scattering of radiation by hot plasmas, and single particle dynamics in magnetic and electric fields. The last category of activity is entirely governed by the interests and inclinations of individual staff members, and thus may vary in scope and direction from time to time.

\section{A. Plans}

The Scyllac-directed work is expected to increase substantially as plans mature. This increase will encompass both man-hours of staff member time and computer use. So far we have investigated numerically only the low beta models of stable confinement; as the unity beta theory develops, we are likely to invest computer time in this area. Theoretical interest in inertial and dynamic confinement is expected to grow, in conjunction with new experiments and ideas. We also hope to encourage and extend investigations in the field of fundamental plasma physics.

\section{ENGINEERING}

The Sherwood engineering effort at Los Alamos has two functions, to provide engineering support for the experimental program and to develop the new components required to build the experimental apparatus. 
Most of the experiments at Los Alamos require very intense, pulsed magnetic fields. Our main engineering effort is directed toward creating these fields with high voltage, energy storage capacitor banks and appropriate coil configurations.

The experiments require capacitor banks operating from 10 - $100 \mathrm{kV}$ which deliver from $100 \mathrm{kA}$ to $25 \mathrm{MA}$ and generate fields from $50 \mathrm{kG}$ to $250 \mathrm{kG}$. We have developed reliable electrical and mechanical techniques for handling these voltages, currents and pulsed magnetic fields.

The Scyllac experiment will use all of our known techniques and require development of several new concepts. New mechanical and electrical engineers are being added to our now modest engineering staff. A prototype sector of Scyllac will be designed and built to evaluate every design and component that will be used in the final machine. When all designs have been approved the final design of Scyllac will be released. Based on considerable past experience the Scyllac development and design will be compatible with our request of FY-68 funding.

The complete electrical design of Scyllac will be done by the Sherwood engineering staff. The final mechanical design, which requires considerably more detailed design and mechanical drawing, will be contracted to an AEC prime contractor, ACF Industries or Sandia Corporation. Both contractors are located in Albuquerque, only 60 airline miles away and both have extensive experience with working intimately with LASL on similar projects.

Scyllac and other proposed pulsed experiments will require development of new energy storage components, principally capacitors and coaxial cables. Since 1956 the IASL Sherwood engineering staff has been working closely with the capacitor industry to develop new and better energy storage capacitors. The $14 \mu \mathrm{F}, 20 \mathrm{kV}$, the $60 \mu \mathrm{F}, 10 \mathrm{kV}$ and $2 \mu \mathrm{F}, 50 \mathrm{kV}$ capacitors are the direct result of this joint effort. This uniquely successful association is being used to develop a reliable $2 \mu \mathrm{F}, 60 \mathrm{kV}$ capacitor for Scyllac. Prototype models are on order from three different manufacturers. They will be evaluated at Los Alamos and the results transmitted back to the individua 1 companies to improve their design. An identical program is under way with the coaxial cable industry. The desired cable is designed and specified by Los Alamos angineers. The design is sent to three different companies which build their version of the design. The cables are evaluated by Los Alamos. The results are then used by the cable companies to design and build reliable cable for our use and many other commercial energy storage applications.

The capacitor and cable developments, for Scyllac have been under way since early 1965. These components will be available for Scyllac when required.

\section{BASIC PLASMA PHYSICS}

The aim of our activities continues to be the study of the fundamental properties of plasmas utilizing both theoretical and experimental means. In the past our research has included a wide variety of topics, and has culminated, to give several examples, in a new theory of the electrical conductivity, an 
important method for producing a steady state highly ionized plasma, and an understanding of the source of the unexpectedly intense emission of microwave radiation by high current discharges. This work led to an understanding of how high energy electrons, called "runaways," can be produced inside of a plasma by an externally applied electric field. Moreover our results indicated that even small deviations from the normal, equilibrium condition in which a plasma was generally assumed to be, could lead to unstable conditions. These instabilities, since termed micro-instabilities, because they first arise on a microscopic level of observation, may constitute a serious obstacle to realization of a thermonuclear reactor. Although a considerable body of theoretical literature regarding these now exists, the experimental check of these predictions remains very incomplete. Much of the work being carried on today to study these instabilities utilizes successfully a plasma device based upon our earlier calculations which indicated that a nearly completely ionized gas could be produced in the steady state by heating Cesium gas in a hot Tungsten enclosure.

Our efforts are presently channeled in two directions. First we hope to put micro-instabilities to work for us by causing these to convert the energy in an electron beam into ion energy. An experiment to test this approach is under construction. Secondly we are working towards an understanding of those plasma mechanisms which can give rise to the loss of plasma energy by its conversion to light waves which can subsequently escape the plasma. For this purpose we have developed a method of calculation which treats these light waves as if they were a gas of particles (commonly called photons) admixed with the electrons and ions of the plasma. This approach has the advantage that the effect which emission and absorption of light has upon the plasma properties can be likened to the usual effects which the collisions between gas particles produce. The application of this technique to a variety of new problems will be continued. We have attacked one of the problems of radiation energy loss with the help of sensitive microwave receivers which detect electron cyclotron and synchrotron radiation emitted by a laboratory plasma. (see Fig. 5). Our measurements so far indicate that the unexpectedly intense emission of such radiation is very strongly correlated with the existence in the plasma of highly absorbing regions (denoted hybrid resonances). Although several theories have been advanced to explain this effect, the phenomenon remains poorly understood, and is thus a potential source of energy loss from thermonuclear reactors as we11. The further study of the mechanism responsible for this radiation, as well as the study of other waves propagating through laboratory plasmas will take much of our effort in the future. A better understanding of plasma dynamics, ways to avoid energy loss, and methods of heating plasma particles can be expected from work of this kind.

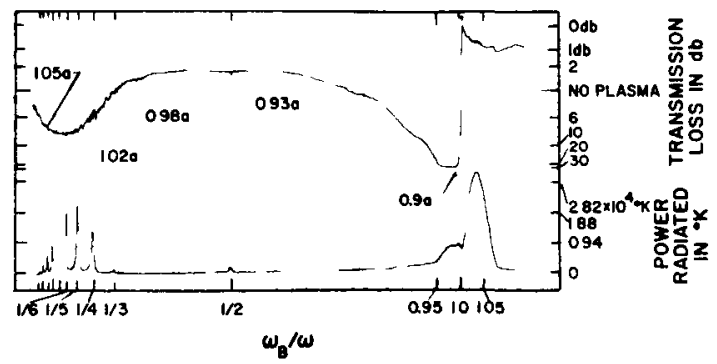

Fig. 5. The transmission loss and power radiated at 18,000 megacycles/sec from a typical laboratory plasma column as a function of magnetic field. 
4. Lawrence Radiation Laboratory

At the University of Califomia Lawrence Radiation Laboratory, a diversified program in controlled thermonuclear research has been pursued since 1952. Three major lines of attack on the problem of production and confinement of a hot plasma, the Magnetic Mirror, Astron, and Toroidal Confinement concepts, constitute most of the effort. In addition, theoretical and experimental investigations of problems in plasma and atomic physics of interest in controlled fusion research are carried out. Several areas of technology have had to be extended in order to meet the conditions required to do significant experiments with hot plasmas. Technological development of considerable variety will continue to be an essential part of the experimental program. In the following three sections, the major lines of attack are described; in the fourth section, some aspects of the basic plasma and atomic physics research are discussed.

\section{MAGNETIC MIRROR PROGRAM}

\section{A. Introduction}

The mirror machine or "open-ended" magnetic bottle not only constitutes one of the most productive methods af understanding the problem of stable plasma confinement, but also shows promise of representing a viable approach to fusion power. The principles of plasma confinement by magnetic mirrors were first conceived and reduced to practice at $I R L$, and the existing Mirror Program is the result of many years of hard work. As a result of this work it appears that a point has been reached where the design and execution of mirror confinement experiments that could answer crucial questions (with respect to stability) can be for the first time based almost entirely on proven technological developments and solid theoretical arguments. For these reasons it appears possible to outline a future course for the LRL Mirror Program that has a high probability of success, in terms of obtaining answers to well-posed physics questions. It is not possible, of course, to guarantee that these answers will spell practical success for open-ended systems, but it is possible to guarantee that the results will be relevant and of general interest.

The present LRL Mirror Program is made up of three mutually-complementary experiments, operating in different plasma regimes, but each aimed at elucidating some aspect of the stability question. These experiments are: 1) Alice, 2) 2X, and 3) Table Top. A key feature of all present IRL mirror experiments is the ability to employ positive-gradient ("minimum-B" fields). The experience at IRL, and that of others, together with the solid theoretical base on which these experiments rest, has led to a growing conviction that for the foreseeable future the advantages of minimum-B fields (in stabilizing gross instabilities) far outweigh any disadvantages they may possess. 
The target for the coming series of mirror experiments is to achieve confined plasmas of high temperature (of order $10 \mathrm{keV}$ ) and of such volume and density as to provide meaningful answers to the stability question. While it is hoped eventuafly to approach steadystate conditions, at plasma densities up to $10^{13}$ or $10^{14}$ ions $/ \mathrm{cm}^{3}$, and plasma volumes of many hundreds of liters, achievement of these conditions is by no means a prerequisite to performing meaningful studies.

\section{B. Components of the Mirror Program}

\section{The Alice Neutral-Injection Experiment}

The advantages of an open-ended confinement experiment in which the plasma is created by the injection and trapping of a neutra] atom beam of high intensity but relatively low energy ( 10 to 20 $\mathrm{keV}$ ) were pointed out, at IRL, in 1958. These advantages were made apparent from a theoretical study of the scaling laws for high energy injection experiments. Shortly after these calculations were made the Alice program was initiated at IRL. Several years preparatory work followed, aimed toward the creation of an intense and highly collimated neutral atom beam (20 keV at 50-75 $\mathrm{mA}$. equivalent current) and toward the achievement (in the presence of such a beam) of confinement chamber vacua of order $10^{-9} \mathrm{~mm} \mathrm{Hg}$. First confinement experiments were begun in 1961, and within a year significant results on plasma stability were obtained. Even though the plasma density first achieved was low (of order 107 ions/ $\mathrm{cm} 3$ ) it was high enough to permit strong cooperative instability effects. It was found that a gross instability, sufficient to limit the plasma density, appeared. This instability was identified with a theoretically predicted hydromagnetic mode and a close correlation with theory was established. Simultaneous with the detection of the low-frequency hydromagnetic modes came the discovery of strong high-frequency instabilities of the "wave-particle" type, occurring at the ion-cyclotron frequency or higher. These observations pointed up not only the existence of high-frequency instabilities in an experiment such as Alice, but showed that such instabilities could appear even in a plasma whose diameter was several ion orbit radii in dimensions.

The next step in the Alice program was to introduce a 12-pole positive-gradient field in order to test in steady-state the stabilizing properties of minimum-B fields. It was found that gross modes were completely stabilized, leading to an increase of peak plasma density by about a factor of 10 (to about $2 \times 10^{8}$ ions $/ \mathrm{cm}^{3}$ ). Though it was found that the application of a 12-pole stabilizing field markedly reduced the high-frequency activity, it did not eliminate it. It was in fact shown that the plasma density was now being limited by small losses associated with this activity. To give an idea of the sensitivity of the loss measurements possible, it was determined that the mean particle lifetime associated with this anomalous loss process 
(the high-frequency instability) was about 0.1 second, thus comparable to the lifetime for the loss of ions by charge-exchange.

Following the test of a 12-bar stabilizing field, an 8-pole stabilizing system, together with other improvements, was introduced. This led to a further increase in density (up to 3 to $4 \times 10^{8}$ ions $/ \mathrm{cm}^{3}$ peak density) and showed an even more marked effect in stabilizing high frequency modes. Now, instead of a saturation in density, the onset of high frequency activity led only to a decrease in the slope of trapped density vs. injected current.

In large part stimulated by the Alice results, a simultaneous theoretical effort has been carried out at IRL in an attempt to understand the instability modes that have been observed, and to trace their origin. One important result of the calculations was to show the importance of achieving a plasma in which all density gradients are "gradual" (as measured in ion orbit radii); i.e., a plasma many orbit radii in radial dimensions. Another result was to show that limitation of the length of the plasma to a dimension of order 300 to 500 orbit radii also contributes to stability. Thus, seemingly, the "ideal" shape for a mirror confined plasma is roughly spherical.

It was also soon apparent from the calculations that one feature of the neutral injection method (as presently employed in these experiments) inevitably leads to an unfavorable stability situation, namely: The use of monoenergetic injected neutral beams, resulting in a trapped plasma with a highly peaked energy distribution, leads to a strong stimulation of high frequency instabilities. The corollary is equally important: Well spread (randomized) distributions should act to suppress such modes. These theoretical results are in general agreement with similar calculations performed in other laboratories; their general validity is rooted in well-founded thermodynamic considerations.

The combined effect of the above experimental and theoretical results led to the initiation of an effort to develop techniques for producing injected neutral beams of wide energy spread. This activity now forms an important element of the Alice program.

Based on both theory and experiment, it seemed apparent that deep, roughly spherical, minimum-B magnetic wells, spread energy injection, and plasmas many orbit radii in radial dimensions together would result in a marked improvement in plasma stability. Therefore, a new Alice coil and vacuum chamber (Alice Baseball I) of unusual design has been constructed. The coil, which uses liquid nitrogen cooled copper conductors, has the general shape of a seam on a baseball, and provides a quadrupole minimum-B magnetic well with a "depth" of almost a factor three in magnetic field intensity (a factor of 
nearly nine in magnetic energy density). The unusual field configuration produced by the Alice Baseball I coil is shown in the attached figure. Both the fieldines (the fan-shaped array of lines) and the contours of constant magnitude of magnetic field are shown. The magnetic well in Alice Baseball I will be the deepest one yet attempted for any neutral injection experiment, as well as the largest, in terms of ion orbit radii. Technical improvements in vacuum, in trapping and in the injected beam have been incorporated that should permit an accessible density almost a factor of 100 larger than present densities. By "accessible density" is meant that density achievable in the absence of instabilities. Though true steady-state operation cannot yet be achieved (coil operation is limited to a few seconds per "pulse"), Alice Baseball I, now in the process of final assembly, should represent a very significant step. If operated with an injected beam of variable energy spread, the experiment should exhibit a marked increase in actual plasma density, and should permit establishing correlations with theories of high frequency wave-particle instabilities.

\section{Alice Experiment - Future Plans}

The course that the Alice neutral injection experiments have taken has been very encouraging. It has stimulated the planning of a sequence of experiments aimed at attacking the remaining scientific questions crucial to open-ended confinement. As now visualized, these experiments would comprise an ascending series, each one of the series representing a closer approach both to the required steadystate plasma density regime ( $10^{13}$ to $10^{14}$ ions $/ \mathrm{cm}^{3}$ ) and to a situation optimized with respect to plasma stability, as predicted by the theoretical calculations or as determined experimentally. It should be recognized, however, that any long-range programmatic projections that are attempted today almost surely will tend to be modified as the research proceeds.

Once the nature of the requirements that theory imposes on a proposed experiment is determined, the rest of the decisions concerning the experiment relate to technological requirements. These decisions have been strongly influenced by another recent development. As a result of work at many laboratories (including IRL) it is now possible to design and construct superconducting magnet coils of almost arbitrary shape and slze, and at flelds in excess of 50 kilogauss at the surface of the conductor. Furthermore, the pace of these developments is so rapid that one can be certain of still further improvements in the near future, towards higher fields and lower costs. At IRL there has already been constructed a small "baseball" 


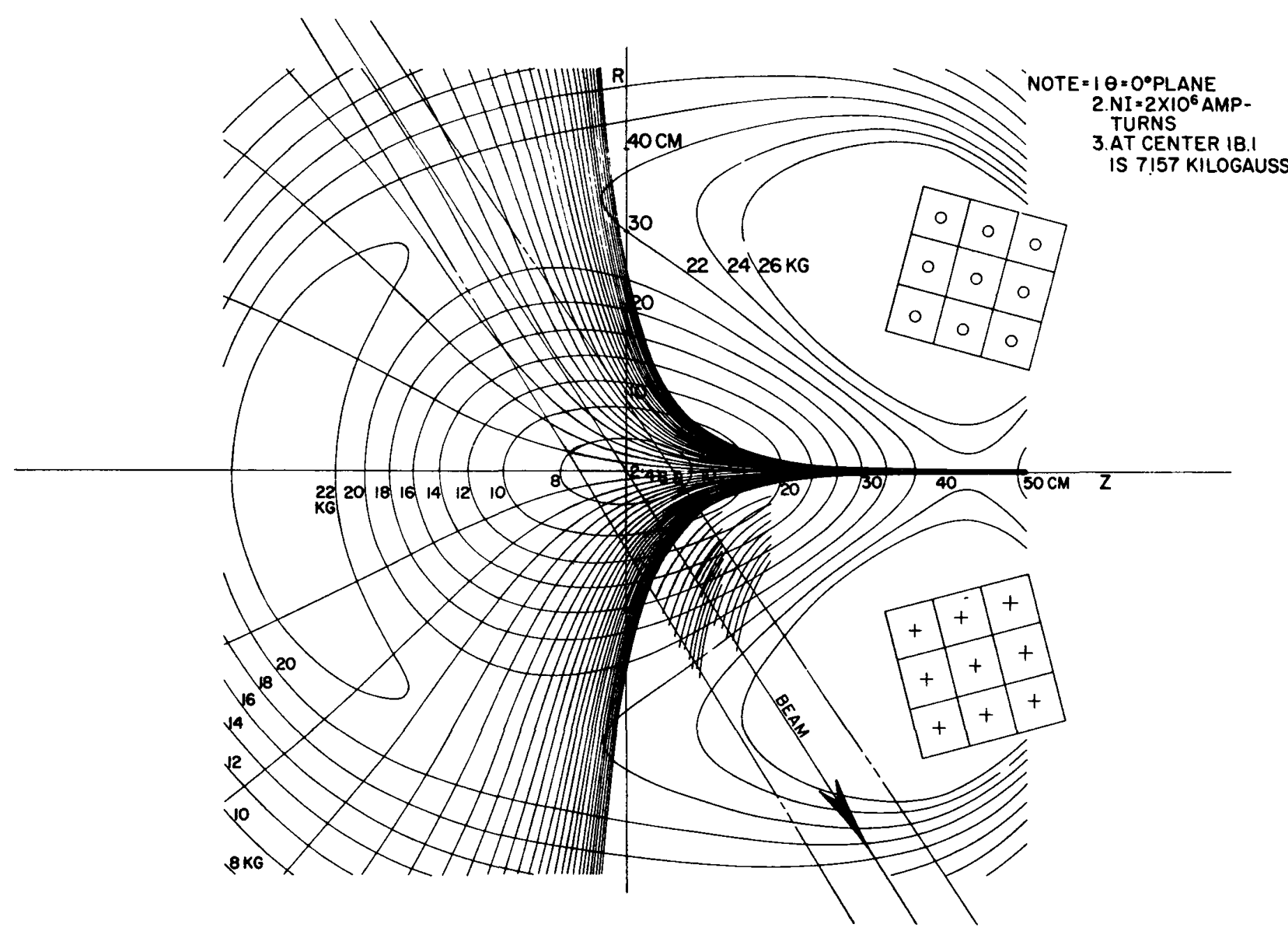

Computed Alice quadrupole magnetic field. Flux lines and contours of constant field modulus are shown in one plane. Field values are for $2 \times 10^{6} \mathrm{~A}$-turns in the coil. The cross-hatched area shows the region of effective Lorentz-force trapping. 
field (Alice"Baby Baseball") using cabled superconducting wire, and its plasma confinement properties are now being tested, using a low current, low energy injected neutral beam.

The most obvious advantage of the superconducting coil is that it produces a high intensity steady-state magnetic field of extreme constancy and at zero power cost. Iess obvious, but of no less importance is the fact that such a coil lends itself to a close-fitted vacuum chamber design where the chamber wall is at supercooled liquid helium temperatures ( $2^{\circ} \mathrm{Kelvin}$, by pumping on the helium). Thus the entire chamber wall becomes an active cryopumping surface. It has been found that the base pressures in such a cryopumped chamber can be one to three orders of magnitude better than presently achieved in Alfce $\left(3 \times 10^{-10} \mathrm{~mm} \mathrm{Hg}\right)$. This possibility opens up a new regime, with important practical consequences: Provided; 1) pressures substantially less than 10-10 $\mathrm{mm}$ Hg can be maintained during the early phases of plasma buildup in an Alice-type system, and 2) injected particle energies that are not too high (i.e., that are less than about $20 \mathrm{keV}$ ) are used, then it should be possible to program the injected neutral beam current so that ion-ion collisions will play an important role in spreading the plasma particle energy distribution. Thus, assuming a modest initial degree of energy spreading is used, maintenance of a low base pressure, together with the use of a programed injected beam current, should permit plasma buildup without appreciable departure at any time from the kind of randomized ion energy distribution required by theory for stability. One promising approach to achieving the required vacuum during the start-up phase is the use of cryopumping, coupled with the use of thin foils (isolating the vacuum chamber) for beam neutralization. All of the above features (superconducting coils, cryopumped chamber, and foil neutralization) are coming under test in the Alice "Baby Baseball."

The above special injection technique and its use to augment the randomization of the plasma is useful mainly at start-up. At higher densities ionization and subsequent scattering of the injected beam by the trapped plasma would become more important, and vacua would be less critical.

On the basis of the above considerations one can visualize one, and possibly two, additional steps in the Alice program that could be accomplished using the present injection and beam handling facility. The first of these, Ali ce Baseball II, would be a scale-up of Alice Baby Baseball, also utilizing a superconducting coil.

Though Alice Baseball II would represent an important step in the sequence of experiments we are outlining, it would still fall short 
of criteria now prognosticated as to the "most probably stable" Alice-type device. Ideally, to achieve yet higher densities, with the least likelihood of instability, it appears that additional measures should be taken. One would be further to increase the radius of the plasma, as measured in ion-orbit diameters. A second would be to introduce means by which the plasma boundary conditions (for example, for the reflection of unstable waves at the mirrors) as well as the shape of the radial density profile of the plasma are both brought under precise control. It appears that these refinements are possible.

To summarize present and near future plans, Table I shows the salient features of the present Alice, the Alice Baby Baseball (under preliminary test), Alice Baseball I (under construction), and Alice Baseball II (projected).

\section{Alice II}

Alice II would be an experiment that could possibly represent a definitive test of open-ended confinement. In such an experiment all of the requirements that it is now believed must be imposed on the nature and the confinement of a mirror-confined plasma in order to achieve high densities in steady-state would be satisfied. From this experiment one could hope to establish particle and energy loss processes with sufficient precision to permit an assessment of the future of open-ended systems. It would be premature today to attempt to establish details of the design of an Alice II, but studies have already shown that it would be of reasonable size.

\section{The 2X Experiment}

The experiment $2 \mathrm{X}$ is an enlarged version of the older "Toy Top" multiple magnetic compression experiment. In the $2 \mathrm{X}$ experiment a large volume (30 liters) of plasma at thermonuclear ion temperatures (5 to $10 \mathrm{keV}$ ) and "practical" densities ( $10^{13}$ to $10^{14} \mathrm{~cm}-3$ ), is produced by plasma injected, transferred to the compression chamber, and subjected to "slow" (200 microseconds) magnetic compression. The plasma density, its temperature and the compression times are so related that the plasma should remain well randomized.

In the past (using Toy Top) the anatomy of a flute instability in simple mirror fields was shown and its partial stabilization by control of the end boundary conditions was demonstrated. The significance of these results, recently corroborated in $2 \mathrm{x}$, was that while fluting can be partially suppressed by the presence of cold plasma, attempts to decrease the density of this cold 
TABLE I

\begin{tabular}{|c|c|c|c|c|c|c|c|}
\hline Experiment & Status & Co11 & $\frac{\text { Pulse }}{\text { Length }}$ & $\frac{\text { Central }}{\text { Eield }}$ & $\underline{\text { Well }}$ & $\begin{array}{c}\text { Plasma } \\
\text { Radius cm }\end{array}$ & $\begin{array}{l}\text { Plasma Radius (in } \\
\text { orbit radii) }\end{array}$ \\
\hline Alice 8-bar & dismantled & $\begin{array}{l}\text { L.N. cooled } \\
\text { copper }\end{array}$ & $1 \mathrm{sec}$ & $7 \mathrm{kG}$ & 1.3 & $12 \mathrm{~cm}$ & 4 (at $20 \mathrm{keV})$ \\
\hline $\begin{array}{l}\text { Alice Baby } \\
\text { Basebal1 }\end{array}$ & $\begin{array}{l}\text { prelim. } \\
\text { operation }\end{array}$ & supercond. & dc & 3.5 & 2.6 & 12 & 6 (at $5 \mathrm{keV}$ ) \\
\hline $\begin{array}{l}\text { Alice } \\
\text { Baseball I }\end{array}$ & $\begin{array}{l}\text { final } \\
\text { assembly }\end{array}$ & $\begin{array}{l}\text { L.N. cooled } \\
\text { copper }\end{array}$ & $5 \mathrm{sec}$ & 7 & 2.8 & 30 & 15 (at $15 \mathrm{keV}$ ) \\
\hline
\end{tabular}

\section{Alice}

Baseball II projected supercond. dc

5. $50 \mathrm{~cm} \quad 50$ (at $15 \mathrm{keV}$ )


plasme so that it did not give rise to other problems (such as charge exchange losses on the accompanying neutrals) result in a reappearance of the flute instability. The resolution of this difficulty is not at present apparent, as long as simple mirror fields are used. It is for this reason that the $2 X$ experiment is equipped with "quadrupole" minimum-B windings.

In earlier experiments, and in the first tests of $2 \mathrm{X}$, vacuum conditions ( and possibly other effects) limited the confinement time (even for flute-stabilized plasmas) to about 200 microseconds. Most recently, some $400 \mathrm{ft} .{ }^{2}$ of liquid-nitrogen cooled "gettered" surface has been incorporated into the vacuum system, dropping base pressures to the 10-9 $\mathrm{mm}$ Hg region, a significant step toward reducing confinement time limitations of environmental origin. The remalning environmental problems are associated with direct bombardment of the walls of the confinement chamber by the injected plasma.

Recently, the $2 \mathrm{X}$ confinement chamber has been equipfed with retractable evaporators. As a result, the hot ion plasma is now observed to survive application of the quadrupole field without interruption or diminution of the prompt neutron flux. These observations, coupled with microwave density determinations, indicate that a reacting deuterium plasma $\left(E_{i} \approx 5 \mathrm{keV}, n_{1} \simeq 5 \times 10^{13}\right)$ was formed, now in minimum-B geometry. In addition to the use of evaporators, wall bombardment was limited by restricting the injected plasma column to a diameter of 6 inches. The quadrupole field was applied 30 microseconds after the mirror compression field was initiated so that all pulsed magnetic flelds reached their maximum value 190 microseconds after plasma injection. Maximum fusion reaction rates were for the first time observed at peak compression and were essentially the same as those observed in simple mirror geometry with line tying (stabilization by "cold" plasma).

With the 6 inch injected plasma column, in minimum-B or simple mirror geometry, the reaction rate decayed with a time constant of 50 microseconds, which is to be compared with the 150 microsecond value reported earlier for the larger diameter case ( 16 ins. diam., simple mirror). If the decay is due in both cases to a particle loss the corresponding characteristic decay times of the density are 100 and 300 microseconds. From microwave data it is known that the density does decay as the reaction rate decreases, however, it is not known if the ions also lose energy before escaping. The dependence of the decay time on radial dimensions is consistent with particle losses, through the mirrors, but at the boundary it could be associated with instabilities arising from radial density gradients. 
Instrumentation is being developed to measure both particle and energy fluxes so that transverse losses can be compared with end losses and their spatial distribution mapped. Such information will help further to identify the loss process.

As the neutral density should be lower with the 6 inch plasma column (as compared with the larger diameter), and since improved wall conditions should have led to longer lifetimes it appears that loss mechanisms other than charge exchange or interchange instabilities are present. The rf signals which are generated in the containment chamber may be indicative of cooperative effects associated with plasma losses. Thus far, the following correlations have been found: In an ungettered vacuum chamber an rf activity in coincidence with a strong neutral atom ( 2 to $4 \mathrm{keV} \mathrm{D}$ atoms) signal occurs 20 to 50 microseconds after a plasma wall contact in the containment chamber. This activity follows a wall contact due to interchange instabilities or plasma diversion by the quadrupole field. The data are consistent with the postulate that at base vacuums of the order of 10-6 torr a plasma wall contact produces a secondary low energy ion group which when combined with the high energy trapped plasma forms a double peaked ionic energy distribution. Such a distribution can result in a loss of perpendicular energy from the energetic ions to the low energy ions via an electrostatic instability.

Intermittent rf bursts, which are seen even in the absence of detected plasma wall contacts, are (within the present limits of measurement) composed of the fundamental and harmonics of the ion cyclotron frequency corresponding to the central magnetic field. Phase correlation of several loop detectors indicates the frequent occurrence of flutelike modes. Again, such modes could be associated with radial density gradient driven instabilities.

It is planned to continue the investigation of the wave-particle instabilities now being observed in 2X. The results could shed much light on the actual effects of such instabilities (for comparison with the predictions of non-linear theory) in a fully developed thermonuclear plasina.

Other experiments now under way in $2 \mathrm{X}$ include examination of the effects of "line tying" under a situation where the results should be relevant to the important question of instabilities in "average minimum-B" systems.

\section{Table Top IV}

Table Top IV is the latest member of a sequence of small, slowcompression, plasma-injection mirror machines at LRL. In this experiment advanced vacuum techniques are used, creating an 
environment ideal for small-scale confinement studies. The present mode of operation produces a "hot electron" plasma, with temperatures of order $10 \mathrm{keV}$ and densities of about $10^{11} \mathrm{~cm}^{-3}$. Residual neutral gas and cold plasma is of much lower density than the hot plasma, facilitating significant stability experiments. Hot electron plasmas are good models for gross instability modes (as encountered in simple mirrors) and the earliest example of flute-stabilized plasma confinement was demonstrated in Table Top. More recently, studies have been made of the anatomy of flute instabilities, and the stabilizing effect of hexapole and quadrupole minimum-B fields has been demonstrated. Hot electron plasmas also provide a good analog for studying high frequency electrostatic instabilities in this case at electron cyclotron frequencies. One of the exciting recent results is the discovery of unstable modes which seem to be of the newly-predicted "loss-cone" variety. Here, the critical scale length, being measured in electron orbit diameters (of order $1 \mathrm{~mm}$ ) is such that even a few centimeters length of plasma suffices. For this reason it should be possible to determine, even with small volumes of plasma, some of the critical features of these types of instabilities and to compare these with theory. Thus these results should be useful as a guide in the execution of the "hot ion" experiments, such as Alice and 2X. In the future Table Top will continue to be used for specific instability studies, using minimum-B or related configurations.

\section{Conclusions}

At the present time, an assessment of three factors: 1) experimental results, 2) present theoretical insight, and 3) recent technological developments, as applicable to magnetic mirror experiments, indicates what appears to be a favorable situation. It appears possible to plan, and to carry out within a relatively few years, a series of experiments that could provide a critical evaluation of openended (mirror) systems as applied to the confinement of thermonuclear plasmas.

It is therefore proposed to initiate, as required, the design and construction of a series of new Alice-type experiments. There is, based on the experimental results to date and on theoretical predictions, good reason to believe that the experimental series should be successful. 
II. THE ASTRON

A. Introduction

The Astron concept represents a unique approach to the CTR problem. High energy electrons are employed to provide both the magnetic bottle to confine the plasma and to produce and heat the plasma to fusion temperature. Energetic electrons are infected and trapped in an evacuated vessel where a magnetic fleld is established by external coils. The vacuum magnetic field is substantially parallel to the axis of symmetry of the evacuated vessel and is constant in time. Therefore, in order to trap the electrons irreversibly, a friction force is required to absorb some of their energy as the injected electron bunch (Fig. I) slides down in the mirror field. A part of the required friction force is provided by resistors located in the walls of the evacuated vessel. By injecting the electrons in short bursts $(.3 \mu \mathrm{sec})$ but at a verr high current ( 100A) it is possible to absorb part of the electron energy through the eddy currents generated in the resistors by the moving electrons.

The infected electrons initially form a thin layer of rotating electrons prescribing helical orbits (Fig. I). The electron layer known as the E-layer, acts itself as a solenoid generating a magnetic field which opposes the field of the external solenoids. By infecting and trapping more electrons, thus bullding up the E-layer, it is expected that the self-field generated by the rotating electrons (within the cylindrical volume enclosed by the E-layer) will become slightly stronger than in the fleld established by the external coils. In this event, the combination of the external field with that of the E-layer will create a magnetic fleld pattern with the lines of force closed within the chamber, as shown in Fig. I. Since in this field configuration no Ines of force escape from the confining region, plasma particles cannot be lost by direct scattering, but only by diffusion across the lines of force. This feature permits an isotropic Maxwellian velocity distribution to persist within the plasma. Plasma trapped within this pattern of magnetic fleld lines tends to enhance the confining magnetic fleld through the Hall currents generated by the pressure gradient in the plasma.

The pattern of magnetic field lines created by the E-layer provides a magnetic fleld increasing outwards, which is generally known as minimum-B configuration and which can confine the plasma in a hydromagnetically stable fashion. In addition, the confinement of the plasma in a pattern of closed magnetic IInes eliminates a priori most of the so called "velocity space" instabilities. This property of the E-layer to provide a true minimum-B field with closed magnetic Iines is unique, for in order to meet this condition it would be necessary to place coils in the plasma. The only colls which can be tolerated in a hot plasma are coils made by charged particles rotating in a magnetic field. Relativistic electrons are by far better than ions for this purpose because of their high velocity and their inherent relativisitic properties allowing focusing and acceleration of very high current beams. Consequently, the E-layer is the only presently envisioned solution to the problem of bullaing a magnetic plasma bottle with true minimum-B and closed magnetic lines. 


\section{THE ASTRON}

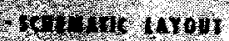

\section{(1)}

(1)

1.

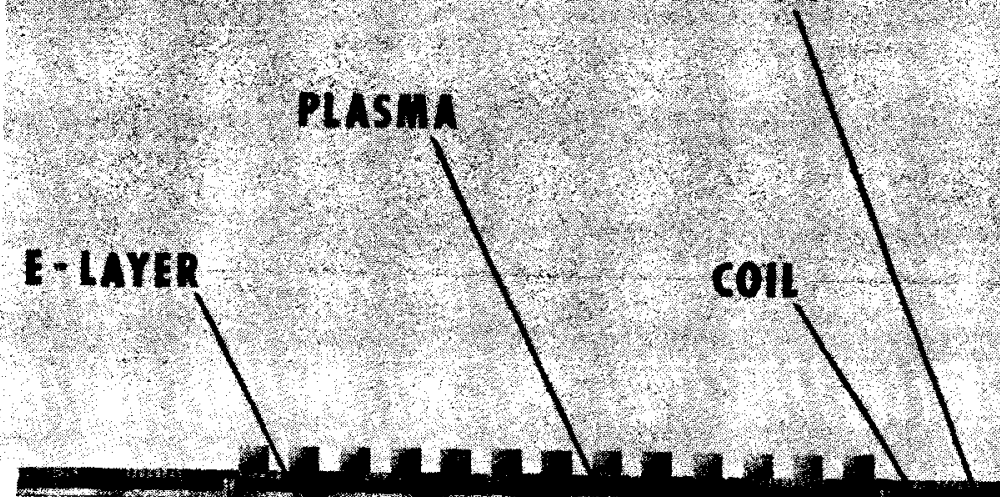

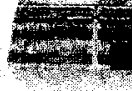
更
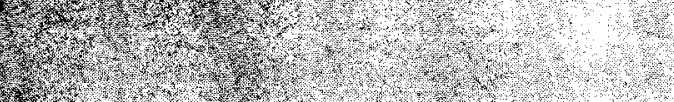

(3t)
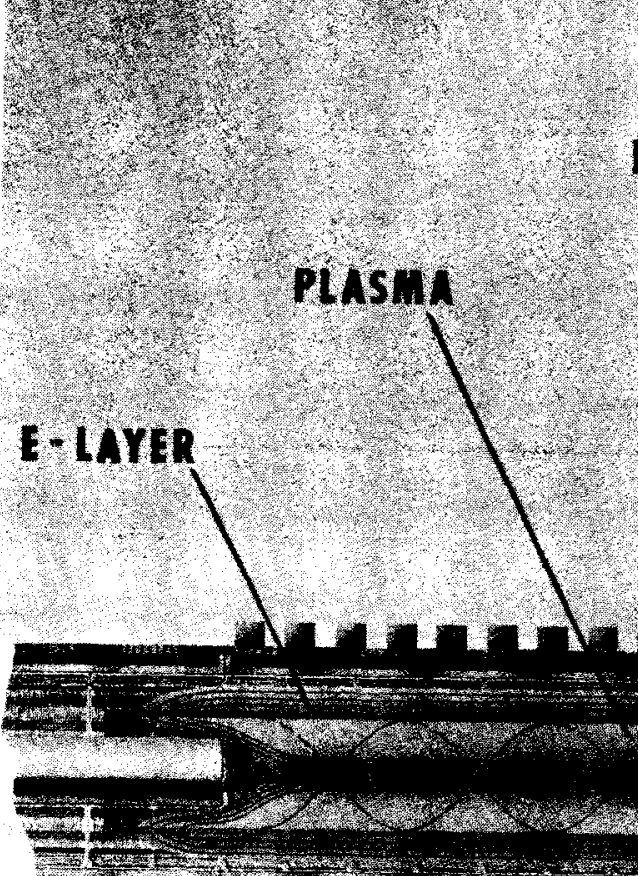

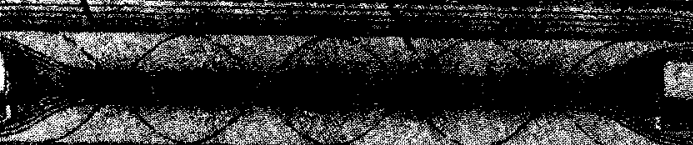
Fistrot

\section{ImDUCTION ICCHEThor \\ (c)}

\section{Erateor con}
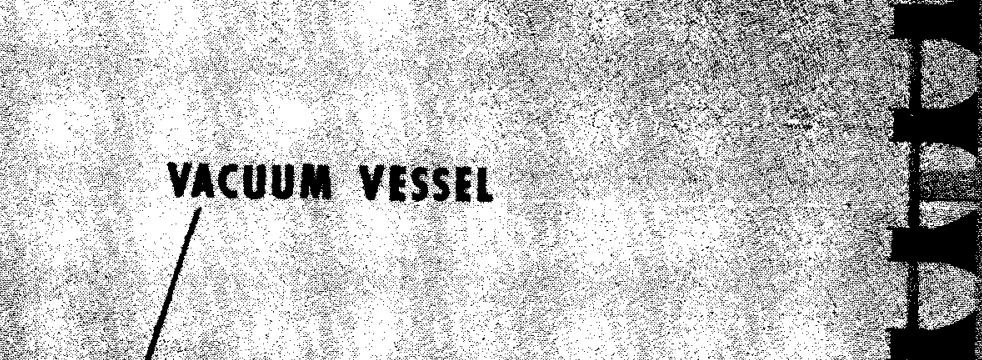

ELECTRON BUNCH 
The Astron concept meets the three known necessary, but maybe not sufficient, conditions to solve the controlled fusion problem, provided however, that the E-layer itself is stable and it behaves like a rigid coil in the presence of dense Maxwellian plasma. The stability of the E-layer itself, which is a prerequisite to the feasibility of the Astron concept, has been questioned because of the anisotropic velocity distribution of the E-layer electrons. The mathematical formulation of the problem based on realistic assumptions is so difficult that no rigorous proof can be provided either to prove or disprove E-layer stability. Therefore, a convincing proof can be provided only by a carefully planned experimental program.

In order to test the Astron concept an experimental facility has been built. The most important component of the facility is the electron accelerator. The parameters are:

$\begin{array}{ll}\text { Electron energy } & 4 \mathrm{MeV} \\ \text { Beam current } & 150 \text { amperes } \\ \text { Pulse duration } & -3 \mu \mathrm{sec} \\ \text { Repetition rate } & 60 \mathrm{pps} \\ \text { Repetition rate for bursts of } 100 \text { pulses } & 1,440 \mathrm{pps}^{*}\end{array}$

* This mode of operation is expected to be available soon.

The accelerated electrons are injected into a tank 90-feet long and 2.5 feet inside diameter. A long solenoid 4-feet in diameter placed outside the tank generates the vacuum field required to guide the injected electrons to form a cylindrical E-layer 2-feet in diameter and 10 to 50 feet long. The walls of the tank are lined with resistors, which slow down the injected electrons.

The cost of the Astron facility is $\$ 7$ million. The total expenditure by the Commision for the Astron program since the program started in 1957 and incluaing FY 1965 is \$9 million.

\section{B. Initial Experimental Operation}

Following eight years of design and construction, operations with the Astron facility began at the end of July 1964. Experiments to date indicate that approximately one-fourth of the infected electrons are trapped irreversibly within the vacuum mirror fiela. During the trapping process, which lasts approximately $\cdot 5$ usec, very strong electromagnetic flelds have been observed. These fields disappear altogether in approximately $1 \mu \mathrm{sec}$ after the injection of the electrons.

The trapped electrons form an E-layer which remains confined for several milliseconds. No velocity space instabilities have been observed to date during the confinement period.

The only measured oscillatory motion, is a low frequency precession of the E-layer. The associated oscillatory magnetic field decays with a time constant 
of several hundred microseconds and occasionally of the order of one millisecond. The decay of the oscillatory magnetic field at a rate faster than the decay of the E-layer indicates that the E-layer is positively stable against low frequency perturbations.

Although the strength of the E-layer to date is only one percent of the required strength to reverse the magnetic field and create the pattern of closed magnet1c IInes, the number of trapped electrons is high enought to demonstrate:

1. The minimum-B configuration required for stable plasma confinement.

2. That a cylindrical layer of relativistic electrons can be created, which behaves like a rigid coll.

3. Overlapping of electron pulses, whereby electrons remain trapped while more electrons are being infected, demonstrating the feasibility of maintaining the E-layer in a steady state.

\section{Future Experimental Program}

All the properties expected of the E-layer have been demonstrated to date except that its strength is limited by the rate of injection of electrons. The next step therefore, is to increase the infection rate substantially by construc ting a new $4 \mathrm{MeV}$ electron accelerator capable of injecting electrons into the Astron tank at such a rapid rate as to build the E-layer up to full strength in less than one millisecond. By employing a high injection rate the present limitations to E-layer build-up will be eliminated except for those which may be imposed by an unexpected violent instability. In addition the proposed new accelerator w1ll be able to provide enough electrons to maintain an E-layer at full strength In the presence of a plasma density of $10^{14}$ ions $/ \mathrm{cm}^{3}$. In the meantime, until the new accelerator is completed experiments will continue with the present accelerator at pulse rates up to $1440 \mathrm{pps}$ for the purpose of improving the efficiency of electron trapping, elucldating further the behavior of the E-layer and studying its interactions with surrounding plasma under a varlety of conditions.

The proposed new accelerator is the first step of the Astron five year program submitted to the AEC in October 1965. The second step is to explore the potential of the Astron concept in the CTR problem. In order to test the E-layer capability of heating to fusion temperature and stably confining a thermonuclear plasma of density $10^{14}$ ions $/ \mathrm{cm}^{3}$ it will be necessary to extend the proposed $4 \mathrm{MeV}$ Astron accelerator to $20 \mathrm{MeV}$. It should be noted that successful completion of the second step of the five year program w1ll have demonstrated the feasibility of the Astron concept as a solution to the CTR problem. 
III. LIVERMORE TOROIDAI CONFINEMEINT RESEARCH

\section{A. Introduction}

Toroldal magnetic confinement eliminates direct end-losses. There remalns the serious problem of plasma loss by anomalous diffusion across field. If the long-wave instability modes can be suppressed, however, one can show that the possibility of cross-field diffusion by residual short-wave modes is not a serlous threat to the realization of a thermonuclear reactor. Present theory indicates furthermore that the long-wave modes can be suppressed in proper confinement geometry. Accordingly, toroldal confinement appears to be a highly credible approach to thermonuclear power.

Thus far most experimental toruses have relled solely on shear stabilization, and have not succeeded in suppressing a rapid cross-field diffusion associated with long-wavelength instability modes ("pump-out"). By way of contrast, the open-ended minimum-B configurations have eliminated cross-field transport, (but are subject to anomalous end-losses).

The LRL Toroidal Confinement Group is approaching the "pump-out" problem by a comparative study of shear-stabllized and minimum-B-stabilized configurations. Since the dangerous long-wavelength instability modes are based on unfavorable geometric properties of the confinement, it is natural to gain understanding and control over them by studying the effect of variations in the confinement geometry.

During the past several years we have made the following contributions to toroidal confinement research:

1. We have discovered the importance of finite-resistivity hydromagnetic instabilities, and have worked out a comprehensive theory.

2. We have shown experimentally both the partial effectiveness of strong shear in reducing instability, and also its inadequacy to eliminate pump-out (In present-day plasmas).

3. We have shown theoretically the adequacy of minimum-average-B configurations to suppress pump-out, and have discovered the existence of minimu-average$B$ configurations suitable for reactor purposes (i.e., without internal "floating rings").

The purpose of our present experiments is the following:

1. We hope to demonstrate in open-ended geometry that point-by-point minimum-B properties are unnecessary, provided that the theoretical minimumaverage $-B$ condition is satisfied.

2. We hope to show that "floating-ring toruses" having strong minimumaverage-B properties will suppress rapid diffusion by pump-out, leaving only short-wave instability modes, if any. 
If our experimental results follow theoretical prediction, our future plan is to construct a minimum-average-B torus without "floating rings", and realize thermonuclear plasma conditions by means of neutral-beam injection.

B. Present Experimental Facilities

We are operating three relatively small facilities, which are designed to be geometrically extremely versatile.

1. The levitron is a torus with one floating ring. Ohmic heating produces plasma of typically $100-\mathrm{eV}$ electron temperature $\left(\mathrm{T}_{e}\right)$ and $10^{12}-3 \cdot 10^{13} \mathrm{~cm}^{-3}$ density $\left(n_{e}\right)$. Microwave heating produces either of two regimes: $T_{e} \sim 50 \mathrm{eV}$ and $n_{e} \sim 10^{12}$ $\mathrm{cm}^{-3}$; or $\mathrm{T}_{e}$ in the $\mathrm{keV}$ range and $\mathrm{n}_{\mathrm{e}}$ in the $10^{9}-\mathrm{cm}^{-3}$ range. In all these regimes, shear-stabilization is found to be helpful, but not sufficient to suppress the plasma pump-out, which takes place in times of approximately $300 \mu \mathrm{sec}$. The levitron has been uniquely suited to apply extremely strong shear in these experiments. A modification 1 s now being made to provide a local (open-ended) minimum-B region in the levitron, which forms part of a larger (closed) minimum-average-B configuration. The object is to produce in the minimum-B region a plasma that is stable against cross-field transport, and then to verify that this property is not lost as the plasma fills out the entire closed minimum-average-B configuration.

2. The stuffed cusp, is an open-ended experiment with plasma similar to that in the levitron. In minimum-B operation, there is no cross-field transport under any conditions. Without minimum-B, there are roughly the same two regimes as in the levitron: $\mathrm{n}_{e} \sim 10^{9} \mathrm{~cm}^{-3} \mathrm{~T}_{e} \sim 3 \mathrm{keV}$, which is highly unstable against crossfield transport; and $\mathrm{n}_{\mathrm{e}} \sim 10^{12^{\mathrm{e}} \mathrm{cm}-3}, \mathrm{~T}_{\mathrm{e}} \sim 20 \mathrm{eV}$, which is made stable by "Iinetylng" of the plasma to conducting chamber walls. The latter result is encouraging, since the phenomenon of stabilization by line-tying to rigid conductors is theoretically the same as the tying of favorable to unfavorable regions in minimumaverage-B stabilization. Modifications in the field configuration are under way to permit the direct testing of open-ended minimum-average-B geometries, without linetying.

3. The alkall-metal plasma facility (Q-Machine) has been used to study the effectiveness of strong shear and line-tying against the "universal instability". Strong shear is found to suppress only the short-wave instability modes. Linetying suppresses all instability. A minimum-average-B configuration is being consructed to confirm that the same degree of stability can be achieved as with linetying.

\section{Summary}

The LRL toroldal confinement work is closely related to the LRL open-ended minimum-B work. We are seeking to eliminate the end-loss problem of the minimum-B configurations by relaxing the point-by-point minimum-B condition, so as to allow toroidal geometry. The achievement of closed minimum-average-B configurations stable against cross-field pump-out will provide us with a solid basis for trapping and long-time confinement of dense hot-ion plasmas. 


\section{BASIC PLASMA AND ATOMIC PHYSICS}

A. Theoretical Research

The theoretical efforts at LRL have encompassed a variety of problems which bear on various phases of controlled thermonuclear research. These efforts are in part directed toward problems of general theoretical interest and in part toward problems motivated by and bearing directly on specific aspects of particular experiments. There is also an active association of some of the theorists with parts of the experimental program. In instances this takes the form of almost dally contact with experimentalists and in other instances collaboration with experimentalists on theoretical and calculational problems.

A comprehensive discussion of the details of these efforts would be unavoidably lengthy so accordingly only the subject matter is outlined. The subject matter falls under the following broad classifications:

\section{Plasma Stabllity Studies}

The extension of the finite Larmor radius stability theory to the nonlinear regime has led to the identification of stability as a consequence of an appropriate symmetry principle. The symmetry principle also gives the necessary and sufficient conditions for linear stability in the case of a static equilibrium. There is a continuing computational study of the hydromagnetic stability of finite pressure plasmas in various minimum-B geometries, both axially and nonaxially symmetric. Universal and interchange instabilities arising from density and electric field gradients including finite Larmor radius effects are being studied. An extensive study of longitudinal electric oscillations in a magnetized plasma at or near the lon-cyclotron-frequency and the relevance to current experiments has been made. These studies all have bearing on the Mirror Machine program. In addition, a particle trajectory code for various magnetic field geometries has been developed for assisting in the analysis of specific experiments.

\section{Relativistic Electron Beams}

The collective behavior of non-relativistic and relativistic particle beams has been studied which includes finite cross section beams interacting with resistive walls, negative mass instabilities, and streaming instabilities. An extensive numerical analysis of the build-up in time of an E-layer continues and extends previous analytical work on the steady-state properties of a more idealized E-Iayer. A detailed analysis of the dynamical behavior of the Astron injection and trapping system has been made. Some of these efforts relate to the Astron experiment and are assisting in the analysis of experimental results.

\section{Miscellany}

There is a continuing effort in atomic physics of interest to controlled thermonuclear research: The application of collision theory to light atom reactions, and the continuing analysis of the phenomena of electric dissociation of 
neutral atoms and molecular ions, which has direct bearing on the high energy Injection devices such as the Alice experiment.

As has been true in the past a considerable fraction of this woris involves extensive machine calculations. The presence at Iivermore of a large variety of modern cormuting machines has been an advantage in solving complex problems, In the reduction of data from experiments and in optimizing the design of experiments.

\section{B. Berkeley Program}

\section{General Philosophy}

During the past five years the Berkeley program has developed a character that may be identified as a support project for the major programs at Livermore. No experiment dealing directly with the containment of hot plasma is being carried out. Instead, the effort can be divided into three categories (although any one activity may belong to several of these simultaneously):

a. Controlled Fusion Auxiliary, such as ion cyclotron heating (terminated December 1963), energetic injection development (Homopolar Gun experiment) and measurement of various atomic cross sections of interest to CTR work.

b. Basic Plasma Research, usually not Involving very high temperature, but leading to improved understanding of plasma behavior in general, and resulting in publications of lasting interest.

c. Student Training Program, providing an opportunity for graduate students at the University of Califormia to develop an interest and gain exper1ence in plasma research, with the expectation that the national effort, will eventually benefit by the availability of physicists specifically trained in plasma physics.

\section{Specific Projects}

\section{a. Energetic Plasma Source}

The major effort in the Berkeley plasma physics program at present consists of a special plasma gun development for injection into mirror machines. The principal purpose here is to explore the possibility of generating an uncontaminated energetic plasma in which a large fraction of the energy is random and transverse to an existing magnetic fleld, and to transfer this plasma along the field lines into a containment region. The method involves a brief but powerm ful radial discharge between coaxial electrodes in the presence of a strong axial magnetic field. The gas is supplied in the form of a short burst so that the neutral background extemal to the main discharge is minimized.

\section{b. Atomic Cross Sections}

Both the facilities and the available talent make the Berkeley site particularly suitable for the study of atomic phenomena of interest in plasma 
physics in general and fusion research in particular. Of the multitude of important examples we name only two that are presently under study:

1) Production rate and lifetimes of excited states of atomic hydrogen and of various species of hydride lons. Some of these species may very well prove useful for high energy injection purposes.

2) Transition probabilities for line radiation of atoms in high stages of lonlzation. Knowledge of these quantities is essential to fusion research both for estimates of power losses by impurity radiation and for spectroscopic diagnostic techniques such as the determination of electron temperatures. In addition these quantities are of fundamental interest in atomic physics and of value in astrophysics.

This entire line of research is excellently suited for participation by graduate students. There seems to be no limit of worthwhile and relatively straightforward work that needs and can be done. It is, therefore, proposed that this activity be continued at the present level, or perhaps even be intensified.

\section{c. Fundamental Studies of Plasma Behavior}

A number of fundamental problems of plasma physics have been under study at Berkeley. As an example, the resistive instability of hydromagnetic equilibria has been investigated with the help of linear sheet pinch discharges. Most of this work utilizes the configuration called "Triax", or tubular pinch, because of Its simplicity and its relative stability at infinite conductivity. Under suitable conditions this pinch indeed showed the tearing instability as predicted by theory if the finite resistivity of the plasma is taken into account. A thorough understanding of such behavior is important for all those approaches such as the Stellarator that involve an initially cold plasma already located in the confinement regions.

\section{d. Fundamental Atomic Physics within Plasma}

Finaliy, it is intended to continue the study of atomic processes in nonequilibrium (recombining) plasmas that has proven so fruitful in the past. The experimental investigation of collisional reactions in plasmas (excitation, Ionization, recombination, spectral line shapes, etc.) are of course of basic interest in themselves. However, a good understanding of many of these processes is also essential for the fusion program inasmuch as they are responsible for important phenomena, such as radiative energy transfer, and because they provide the basis for many very useful diagnostic techniques. 


\section{$\underline{\text { Appendix } 4}$}

Washington-Designated AEC Contracts, December, $1965^{*}$

1. Experimental Hot Plasma Studies

Johns Hopkins University

Applied Physics Laboratory

Massachusetts Institute of Technology

Stevens Institute of Technology

United Aircraft Corporation

Westinghouse Electric Corporation

Wisconsin, University of
Theta-Pinch Plasma Gun

Study of Radio Frequency Emission from Hot Electron Plasmas

Experimental Investigation

Cusped Containment Geometries

Investigation of the Production of Plasmas for Thermonuclear Research by Laser Irradiation of Solid Particles

Research to Produce a Plasma by the Use of a High-Power Laser on Condensed Material

Studies of the Trapping, Stability and Characteristics of Plasma in Toroidal Multipole Magnetic Fields

2. Experimental Cold Plasma and Diagnostic Studies

California, University of

Johns Hopkins University

Maryland, University of

Maryland, University of
Plasma Instability Experiments

Plasma Instabilities and Waves Excited by Electron Temperature Anisotropy Produced by Electron Cyclotron Resonance

Applications of Light Scattering to Plasma Diagnostics

Investigation of Universal Plasma Instabilities and Nonlinear Mechanics of Unstable Plasmas

* Details of this work are provided as an attachment to the present document and have also been published separately by the Atomic Energy Commission as TID-4005 (Pt. 5), 1965. 
Massachusetts Institute of Technology

Maksachusetts Institute of Technology

Miami, University of

National Bureau of Standards

Stanford University

Stanford University

Texas, University of

3. Theoretical Plasma Studies

California, University of

Michigan, University of

New York University

Stevens Institute of Technology

Tennessee, University of

Texas, University of

Yale University
Plasma Physics, Plasma Production, and Plasma Diagnostics Studies

Nonadiabatic Trapping of Particles in Magnetic Fields and the Interaction of Optical Radiation with Plasmas

Instabilities and Turbulence in Alkalj Vapor Plasmas

Plasma Density Correlation and Diffusion

Research on Plasma Oscillations and Instabilities

The Study of Anomalous Cross-Field Diffusion

Investigations of the Hybrid ElectronIon Resonance

Theoretical Research Program in Plasma Physics

Theoretical Study of Microinstabilities in Inhomogeneous Plasma

Plasma Physics and Magnetofluid Dynamics

Investigation in Plasma Dynamics

Instabilities due to Anisotropic Velocity Distributions

Anomalous Diffusion and Thermalization of Turbulent Plasmas

A Review of the Kinetic Theory of Waves in a Plasma in an External Magnetic Field 
4. CROSS SECTIONS

Georgia Institute of Technology

Georgia Institute of Technology

National Bureau of Standards

5. VACUUM AND WALL STUDIES

National Bureau of Standards

National Bureau of Standards

Stanford Research Institute

Washington State University

Westinghouse Electric Corp.

6. MAGNETIC FIELD DESIGN

Swarthmore College
Ionization, Excitation, and Charge Transfer Cross Sections

The Excitation of Alkali

Ions by Electron Impact

Crossed Beam Studies of Positive Ions of Molecular Hydrogen

Photo-electric Properties of Solids in the Far

Ultra-violet

Surface Studies of the Deposition of Hydrogen

Beams on Metals

Interaction of Hydrogen and Other Gases with Clean Metal Surfaces

Chemical Sputtering of Solids

Surface Physics and UltraHigh Vacuum Techniques

Axially Symmetric Iron-Free Systems to Produce or Measure Magnetic Fields and Field Gradients 


\section{Appendix 5}

Glossary of Fusion Terms

$\underline{\text { Term }}$

adiabatic compression

afterglow

Alfvén waves

ambipolar diffusion

$\operatorname{arc}$

atomic number

(z-value)

\section{Definition}

1. A compression (of a gas, plasma, etc.) which is not accompanied by the gain or loss of heat from the outside.

2. For a plasma in a magnetic field, a compression which is slow enough that the magnetic moment (and other adiabatic invariants) of the plasma particles may be taken as constant.

The radiation emitted from a plasma after the source of ionization (discharge current, etc.) has been removed. When the plasma temperature has become sufficiently cool, the free electrons recombine with the ions, resulting in the emission of recombination radiation.

Waves occurring in a plasma immersed in a magnetic field, characterized by a transverse motion of the lines of force together with the plasma particles. These transverse hydromagnetic waves propagate at a velocity which depends on the strength of the magnetic field and the particle density.

A diffusion process in which, due to the presence of space-charge fields, the electrons and ions escape to the walls at exactly the same rate. These space-charge fields are selfgenerated within any plasma and act to preserve a close approximation to charge neutrality.

An electric discharge between two electrodes.

A number corresponding to the number of protons in the nucleus (or, in a neutral atom, to the number of external electrons). It is thus an integer expressing the nuclear charge in units of the electronic charge $e$. 
astron

$\beta$-value

bremsstrahlung

burnout

charge exchange

cooperative phenomena

coulomb collision

coulomb force
The name given to a specific concept in the field of controlled fusion. The confining field, which is closed on itself in cylindrical geometry, results from the superposition of an externally gene rated axial field and an opposing field arising from a confined annular layer of relativistic electrong (the so-called E-layer). Cold neutral gas admitted into the vessel will be heated by energy transfer from the E-layer electrons.

The ratio of the outward pressure exerted by the plasma to the inward pressure which the magnetic confining field is capable of exerting.

The radiation emitted as a result of the deflection (e.g., through collisions) of rapidly moving charged particles.

A term applied to the rapid reduction in the density of neutral particles in a discharge, as a result of their being ionized more rapidly than they are formed.

A process in which there is a transfer of charge between two bodies during a collision between them (e.g., the collisional transfer of an electron from a neutral atom to a singly charged positive ion, the latter becoming neutral and the former charged).

The motion of interacting particles acting collectively, rather than individually le.g., plasma oscillations, turbulence, instabilities of one type or another).

A collision between two charged particles. The interaction of their electric fields results in a deflection of each of the particles from its initial path.

The force of repulsion (or attraction) exerted by one electrically charged body upon another. Also called electrostatic force. 
Term

critical temperature

cross section

(for a given event)

cusped geometry

cyclotron frequency

cyclotron radiation

Debye length

degenerate configuration
Definition

The temperature at which the energy generated in a plasma through the fusion process just equals the energy losses (e.g., through radiation processes). (Also called the ignition temperature.)

A quantity proportional to the probability that such an event will occur per unit time.

A magnetic configuration in the torm of cusps, such that the lines of magnetic force are everywhere convex toward the center of the configuration. Such a configuration is of particular interest for the confinement of plasma, since it is theoretically stable against the development of hydromagnetic instabilities.

The natural frequency of gyration of a charged particle in a magnetic field. Often, and incorrectly, called the Larmor frequency. (The gyration frequency is twice the Larmor frequency of precession of an orbiting electron in a magnetic field.)

The radiation emitted by charged particles in a magnetic field as a result of their natural gyration in that field. The particle gyrates at the cyclotron frequency. Sometimes called synchrotron radiation, especially for very fast particles.

A characteristic length in a plasma, corresponding to the distance over which an electron will be influenced by the electric field of a given positive ion. In effect, it is a measure of the distance over which the electron charge density can differ significantly from the ion charge density.

A configuration in which the magnetic lines of force close exactly on themselves after passing a round the configuration a finite number of times. 
$\underline{\text { Term }}$

deuterium atom

deuteron

diagnostics

diamagnetic effect

diffusion

divertor

Doppler broadening

drift surface

\section{Definition}

An isotope of the hydrogen atom with one proton and one neutron in its nucleus, and a single orbital electron.

The nucleus of a deuterium atom.

The procedure of determining (diagnosing) by one means or another exactly what is happening inside an experimental device during an experiment. Also the instruments used for diagnosing.

The tendency of a magnetic field interacting with a system to be reduced in intensity as a result of generation of circulating currents within the system.

The interpenetration of one substance into another as a result of thermal motion of the individual particles (e.g., the diffusion of a plasma across a magnetic field as a result of collisions).

A component of a stellarator which serves to divert charged particles in the outer shell of the discharge into a separate chamber where they strike a barrier, become neutralized, and are pumped away. In this way, the energetic particles in the outer shell are prevented from striking the walls of the main discharge chamber and releasing secondary particles which would cool the discharge.

The broadening of a spectral line as a result of the motion of the emitting atoms toward or away from the detecting equipment. The magnitude of the broadening can be used to calculate the temperature of the emitting atoms.

A surface on which the guiding center of a particle is constrained to move, under the laws of adiabatic invariance. 
$\underline{\text { Term }}$

electron volt (eV)

energy balance

energy replacement

time (or energy loss time)

entropy trapping

excitation radiation

Fokker - Planck equa tion

fusion

fusion, controlled thermonuclear
A unit of energy, equal to the energy acquired by a singly charged particle in passing through a potential difference of one volt. $1 \mathrm{eV}=1.60 \times 10^{-12} \mathrm{erg}$.

A comparison of the energy input into a system (a hot plasma, say) and the energy dissipated by one mechanism or another (e.g., by an increase in the plasma temperature, by radiation, or by various mechanisms of particle loss from the plasma).

The time required for a plasma to lose (via radiation or other loss mechanisms) an amount of energy equal to its average kinetic energy.

The trapping of an ordered beam of particles in a magnetic field configuration (e.g., a cusped geometry) through the process of randomizing the ordered motion of the particles, with a resultant increase in the entropy of the system.

The radiation of line spectra as a result of the excitation of excited states (e.g., through inelastic collisions of electrons with ions) and the subsequent de-excitation of these states by radiative transitions.

An equation which describes the motion of a free particle in velocity space, and which is applicable to plasmas when the cumulative effect of weak deflections resulting from relatively distant encounters is more important than the effect of occasional large deflections.

The process in which nuclei undergo nuclear fusion reactions.

The process in which very light nuclei, heated to high temperature in a confined region, undergo fusion reactions under controlled conditions, with the associated release of energy which may be harnessed for useful purposes. 
$\underline{\text { Term }}$

hard-core pinch device

hydromagnetic instability

hydromagnetics

ignition temperature

instability, hydromag • netic

instability, interchange

instability, kink

instability, twostream
Definition

A pinch-discharge device which incorporates a solid central conductor. The discharge occurs in an annular region about this conductor.

(See instability, hydromagnetic.)

(See magnetohydrodynamics.)

(See critical temperature.)

An instability arising from the macroscopic motions of a conducting fluid (liquid or gas) as a result of its interaction with a magnetic field. Examples are the interchange instability and the kink instability.

The name given to the type of hydromagnetic instability in which the plasma interchanges position with the magnetic field. Also called a "flute-type" instability, since it would be expected that the interface between the plas$\mathrm{ma}$ and the magnetic field would become fluted.

A hydromagnetic instability which sometimes develops in a thin plasma column which is carrying a strong axial current. If a kink begins to develop in such a column, the magnetic forces on the inside of the kink become larger than those on the outside, so that in general it tends to grow in magnitude. The column then becomes unstable and undergoes a gross lateral displacement toward the walls of the discharge vessel.

An instability which can develop when a stream of particles of one type has a velocity distribution with its peak well separated from that of another type of particles through which it is flowing. A stream of energetic electrons passing through a cold plasma can, for example, excite ion waves which will grow rapidly in magnitude at the expense of the kinetic energy of the electrons. 
$\underline{\text { Term }}$

instability, universal

interchange instability

ion

ionization

ion cyclotron resonance heating

isotope

joule heating

kink instability

Kruskal limit

Landau damping

\section{Definition}

A low-frequency instability resulting from the presence of density gradients perpendicular to the magnetic field lines. An instability of this type is generally localized and usually has a small rate of growth.

(See instability, interchange.)

An atom (or molecularly bound group of atoms) which has become charged as a result of gaining or losing one or more orbital electrons. A completely ionized atom is one stripped of all its electrons.

The process of removing an electron from a neutral atom, thereby creating an ion. The term is also often used in connection with the removal of an electron from a partially ionized atom.

The heating of a plasma by the resonant absorption of energy from waves induced in the plasma at or near the ion cyclotron frequency.

One of several species of the same element, possessing different numbers of neutrons (but the same number of protons) in their nuclei.

(See ohmic heating.)

(See instability, kink.)

A value of current which, if exceeded, should theoretically result in hydromagnetic instabilities in the stellarator.

The damping of a space charge oscillation by a stream of particles moving at a velocity which is slightly less than the phase velocity of the associated wave. In this process, the axial velocity of the particles is increased slightly at the expense of the amplitude of the oscillation. 
$\underline{\text { Term }}$

Langmuir probe

Lorentz dissociation

Lorentz gas

Lorentz ionization

magnetic confinement

magnetic mirror
Definition

A small metallic conductor which is often inserted into a plasma in order to measure some of its properties (e.g., density, temperature).

The dissociation of molecular ions by the mechanism described under "Lorentz ionization."

A hypothetical gas in which the electrons are assumed not to interact with each other, and all the positive ions are considered to remain at rest. (Also called an electron gas.)

The ionization of neutral atoms (usually to highly excited states) by injecting them at high velocity $(\bar{v})$ into a strong magnetic field $(\bar{B})$. The atom experiences forces exerted in opposite directions on the positive and negative charges which tends to ionize the injected atom.

The confinement of plasma within a limited region by a magnetic field so arranged that the charged particles of the plasma which tend to leave the region are turned back into the region.

A magnetic field which is generally axial with a local region of increased intensity causing convergence of the field lines. A particle moving into the region of converging magnetic field lines will be reflected if the ratio of its energy parallel and perpendicular to the magnetic field satisfies the relationship:

$$
\frac{E_{\|}}{E_{\perp}} \leq\left(\frac{B_{m}}{B_{O}}-1\right),
$$

where $B_{m}$ and $B_{o}$ are the magnetic field strengths at the mirror and at the original point, respectively. 
Term

magnetic mirror

machine concept

magnetic pressure

magnetic pumping

magnetohydrodynamics

magnetohydrodynamic waves

Maxwell-Boltzmann distribution

mean free path (for a given event)

minimum-B configura tion

mirror ratio

\section{Definition}

A specific concept under investigation in the field of controlled fusion. The plasma is confined in a straight tube by means of an externally imposed axial magnetic field, with magnetic mirrors at each end. Particles are injected when the field is low, and are trapped and heated adiabatically as the field strength is rapidly increased.

The pressure which a magnetic field is capable of exerting upon a plasma.

A term given to a type of plasma heating in which the plasma is successively compressed and expanded by means of a rapidly fluctuating external magnetic field.

The science dealing with the motion of electrically-conducting fluids (liquids and gases) interacting with a magnetic field. (Also called hydromagnetics.)

Material waves in an electrically conducting fluid in the presence of a magnetic field.

The distribution of particle velocities (or energies) which occurs in any gas or plasma when it is in thermal equilibrium at a given temperature.

The distance a particle will travel, on the average, before undergoing such an event.

The name given to a magnetic configuration which increases everywhere in strength with increasing distance from the plasma which it is confining. In such a configuration, the plasma finds itself in a region of minimum magnetic potential.

In a magnetic mirror configuration, the ratio of the strength of the magnetic field at the strongest point on its axis to that at some other axial point (usually taken to be the point of weakest field strength between two magnetic mirrors). 
Term

mobility

molecular ion injection concept

neutral injection concept

neutralized plasma

ohmic heating

pinch effect

plasma
Definition

The equilibrium drift velocity attained by a charged particle when subjected to acceleration by a unit electric field and to the opposing frictional force of collisions with other particles.

A concept under investigation in the field of controlled fusion, in which energetic molecular ions are injected into a suitable magnetic container and are dissociated therein by any of several processes (e.g., collision with neutral atoms, Lorentz dissociation, etc.). The density of trapped energetic atomic ions is then built up to values of the rmonuclear interest, with the simultaneous conversion of their directed velocities into the random motion of a hot plasma.

A concept similar to that described under "molecular ion injection concept," but with the molecular ions replaced by fast neutral atoms which are subsequently ionized inside the magnetic container.

A plasma with no net charge.

The heating resulting from the resistance which a medium offers to the flow of electric current. Often called joule heating. In a plasma subjected to ohmic heating, the ions are heated almost entirely by transfer of energy from the hotter electrons.

The constriction of a plasma column carrying a large current, due to the interaction of that current with its own (encircling) magnetic field.

An ionized gaseous system, composed of an electrically equivalent number of positive ions and free electrons, irrespective of whether neutral particles are present or not. In view of its abundance in the universe, it is sometimes called the fourth state of matter. 
$\underline{T e r m}$

plasma frequency

Project Sherwood

pumpout

rotational transform

"runaway" electrons

scattering

shear fields

\section{Definition}

The natural frequency of oscillation of a plasma, due to the collective motion of the electrons acting under the restoring force of their space-charge attraction to the relatively stationary ions. This frequency is proportional to the square root of the electron density.

The name often used to designate the U.S. program in controlled fusion.

A name given to the anomalously high loss of particles to the walls in stellarator discharges - a loss rate which is substantially in excess of that expected from normal classical diffusion processes.

A magnetic field configuration is said to possess rotational transform if the lines of force, after one circuit around the configuration (e.g., a torus), do not close exactly on themselves, but are rotated through some angle about the so-called magnetic axis.

Those electrons in a plasma which gain energy from an applied electric field at a faster rate than they lose it through collisions. Since the collision cross section decreases as the velocity increases, these electrons tend to "run away" in energy from the remainder of the plasma.

The deflection of one particle as a result of collision with another. Elastic scattering is a scattering process in which the total kinetic energy is unchanged.

In the sense used in plasma physics, magnetic fields having a rotational transform which changes with radius; e.g., in the stellarator tube, magnetic fields that increase in pitch with distance from the axis. 
Term

sheath

shock heating

shock wave

stellarator concept

symchrotron radiation

temperature. kinetic

thermonuclear conditions

\section{Definition}

The region of transition between a neutral plasma and a solid surface in contact with it. While the plasma itself is nearly an equipotential region, there exists a strong potential gradient within the sheath itself, and electrical neutrality in that region is not preserved.

The heating produced by the impact of a shock wave.

The wave produced (e.g., in a gas) as a result of a sudden violent disturbance. To produce a shock wave in a given region, the disturbance must take place in a time short compared with the time required for sound waves to traverse that region.

The name given to a specific concept in the field of controlled fusion, involving confinement of the plasma in an endless tube by means of externally imposed magnetic fields which are essentially axial but which provide, by one means or another, the rotational transform which is essential for plasma equilibrium in this configuration.

(See cyclotron radiation.)

A measure of the energy of random motion of an assembly of particles in thermodynamic equilibrium. Specifically, it is the temperature ( $T$ ) appropriate to the Maxwellian dis tribution assumed by a system of particles upon equipartition of energy among the three translational degrees of freedom. The mean particle energy is then $3 / 2 \mathrm{kT}$, where $\mathrm{k}$ is Boltzmann's constant.

The achievement of an adequately confined plasma having temperature and density sufficiently high to yield significant release of energy from fusion reactions. 


\section{Term}

tritium atom

turbulence

two-stream instability

unipolar arc

universal instability

$z$-value

\section{Definition}

An isotope of the hydrogen atom with one proton and two neutrons in its nucleus, and a single orbital electron.

Violent macroscopic fluctuations which can develop under certain conditions in fluids and plasmas and which usually result in the rapid transfer of energy through the medium.

(See instability, two-stream.)

An arc between a metal surface and a plasma in contact with it. Such an arc requires only one electrode and is maintained by the thermal energy of the electrons.

(See instability, universal.)

(See atomic number.) 


\section{APPENDIX 6}

COMMNTS OF THE GAC AND PSAC ON THIS POLICY AND ACTION PAPER

Comments of GAC:

Dr. Bishop gave the Committee an excellent summary of the status of controlled thermonuclear research, the recommendations of the Herb Panel and the AEC staff's proposals for policy and action in this field.

The Committee is pleased to note that both the Herb Panel and the AEC staff paper stress the need for better coordination of CTR at the project laboratories. The advisory committee which Dr. Bishop plans to appoint, with membership made up of CTR Project Directors from the four 1aboratories supplemented by four well-qualified and highly respected experts from universities and industry, will be an effective vehicle for providing the required coordination. We are confident that, under Dr. Bishop's direction, coordination and cooperation among the laboratories will be improved, and that he will exercise dynamic leadership in obtaining the support of the members of this advisory committee in the difficult decisions he will have to make.

We consider that the Commission has been very fortunate to obtain Dr. Bishop's services as Director of the Controlled Thermonuclear Research Program, and we urge the Commission to give Dr. Bishop the fullest possible support in carrying out this program.

We endorse the recommendations of both the Herb Panel and the AEC staff paper that the AEC "expand the support of these (plasma research activities at universities) at a more rapid rate than that of the National Laboratories." We believe that the AEC has not made use of Universities to a sufficient extent in conducting the more fundamental research needed in this program or in training the next generation of investigators who will be needed to contribute new ideas to it.

We concur in the assessment of the Herb Panel and the AEC that this is an important field and one in which the United States should play a leading role. We are not prepared to recommend, however, that the U.S. should strive necessarily to outstrip all other nations in this field.

We are glad to note that both the Herb Panel and the AEC staff paper recognize the need for "studies of a basic nature in the broader aspects of the science and technology of plasmas."

However, the policy statement of the AEC staff paper to the effect 
that the U.S. program in this field will continue to be directed primarily toward the eventual achievement of controlled

thermonuclear power seems too narrow. We recommend that the policy statement be rephrased to read that the U.S. program in this field will continue to be motivated by interest in eventually achieving controlled thermonuclear power and that the program will emphasize studies of a basic nature in the broader aspects of the science and technology of plasma. Progress in this field in the past has been delayed by attempting to push too rapidly toward the goal of thermonuclear power without taking the time to develop the requisite knowledge of plasmas. Moreover, other valuable applications besides thermonuclear power $c$ an be anticipated from a substantial plasma research program. The increasingly favorable prospects for fast breeders also make thermonuclear power a less urgent objective.

The Committee concurs with the recommendations of the AEC staff paper that the number of scientists and engineers in the program be augmented and that the work underway both in existing laboratories and in off-site installations be intensified. We agree also that major new experiments need to be undertaken. This might be done at the rate of one every year or so. To do all this will require a substantial increase in the AEC's budget. At the same time, we do not believe that the urgency of this work is so great as to justify a total increase in the budget greater than the $15 \%$ per year for five years recommended by the AEC staff paper. With such a control on funds, it will not be possible to do everything which every interested group would favor. To obtain the maximum benefit under these conditions, Dr. Bishop and the AEC must be highly selective in the choice of projects to be supported and should be prepared to eliminate activities which are of declining value so as to release funds for the new facilities, projects and personnel which are needed to provide vitality for the program.

Before deciding to establish a new National Center for Plasma Studies and Controlled Fusion, Dr. Bishop should be given ample opportunity to reorient, coordinate and streamline the present plasma efforts of the project laboratories and other participants in the AEC's program. These groups, working together, should be able to achieve satisfactory progress without the radical and expensive measure of establishing a new National Center. 
Honorable Glenn T. Seaborg

Chairman

U.S. Atomic Energy Commission

Washington, D.C.

Dear Glenn:

Thank you very much for your letter of May 11, 1966, in which you enclosed excerpts of the conclusions of the General Advisory Committee on the Controlled Thermonuclear Research Program of the Atomic Energy Commission.

As you know, the President's Science Advisory Committee has also discussed the program. In general, its conclusions are quite similar to those of the General Advisory Committee.

Sincerely,

Donald F. Hornig

Special Assistant to the President

for Sclence and Technology 
Functions of the CTR Standing Committee

I. Background.

A. The AEC's Controlled Thermonuclear Research Program consists of the activities of a number of scientific and technical groups whose work is (to varying degrees) mutually interdependent. In view of the limited amount of total funding available, it is essential that this interdependence be recognized and that coordination and cooperation between personnel in the program be sufficient to insure that what is eventually pursued has been duly considered by others in the program with related interests.

B. To assist in getting the desired cooperation, it is planned to set up coordinating activities on two levels within the overall program:

1) A CTR Standing Committee, consisting of the Assistant Director (for Controlled Thermonuclear Research) of the AEC's Division of Research, the Laboratory Project Directors and several prominent scientists, to ensure a close cooperative effort within the overall program and to provide guidance on major policy decisions.

2) Ad Hoc Working Panels, consisting of scientists assembled for a limited period of time to study specific programs for extensive research efforts in specialized areas within the CTR program. These Panels will assist the Standing Committee.

II. Charter of the CTR Standing Committee.

A. The CTR Standing Committee would be composed of the Assistant Director (for Controlled Thermonuclear Research), the CTR Project Directors from each of the major laboratories, and prominent U.S. scientists selected from the scientific community as a whole.

B. The function of this committee would be to advise and guide the Controlled Thermonuclear Research Program of the AEC's Division of Research on a continuing basis by:

1) reviewing the areas in which plasma physics and controlled thermonuclear research are conducted under the authority of Section 31 of the Atomic Energy Act of 1954 , as amended. 
2) assessing the overall balance among the various elements in the $\mathrm{AEC}^{\prime} s$ controlled thermonuclear research program, as viewed in the context of national and world plasma physics research.

3) evaluating the scientific significance and productivity of elements of the program and defining the high priority and low priority elements.

4) Identifying major questions requiring immediate attention and research areas critical to the success of the current and future program.

5) recommending how available and projected funds can most effectively be used to carry out the Commission's CTR program.

III.Charter of the Ad Hoc Working Panels.

A. The AEC CTR Office will from time to time, request certain groups to prepare formal documents describing their program plans, rationale and projected costs in sufficient detail to be suitable for program-wide review. To conduct this program-wide review an Ad Hoc Working Panel would be assembled, consisting of one representative from each of the four major CTR laboratories (appointed by the Project Director) and several other representatives from outside those four laboratories (appointed by the AEC Program Director).

B. The goal of the Working Panel would be to review a specific planned program in a cooperative and constructive spirit, to ensure that the scientific and technical basis is as sound as possible. The functions of the Working Panel would include:

1) Scientific and technical analysis during which the members would request whatever assistance they required from qualified staff at their home 1aboratories.

2) Consideration of similar research already performed elsewhere, to the extent that such research affects the scientific objectives under review.

3) Attempts, via discussions, to pursuade those concerned with the specific program to give due consideration to objective and constructive criticisms. Hopefully this would often result in program modifications even during the course of the review. 
4) Preparation of a report covering such items as the salient features of the critical analysis, changes which have resulted in the original plans, recommendations concerning the adequacy of the proposed program and concerning the sclentific desirability of proceeding in the manner and at the rate proposed.

C. The functions of the Working Panel would not include:

1) Making criticisms of a purely subjective nature.

2) Making recommendations concerning the relative merits of performing research in different areas (e.g., mirror research vs. theta pinch research), except insofar as it was determined that the specific scientific goals could be achieved more logically by methods other than those proposed. 